Nevada

Environmental

Restoration

Project

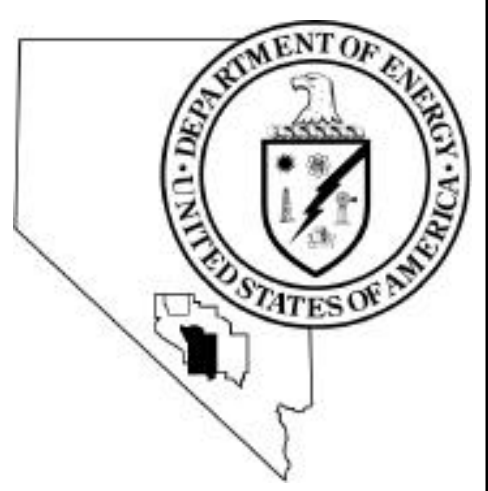

Corrective Action Decision

Document/Closure Report for

Corrective Action Unit 266:

Area 25 Building 3124 Leachfield,

Nevada Test Site, Nevada

Controlled Copy No.:

Revision No.: 0

February 2000

Approved for public release; further dissemination unlimited.

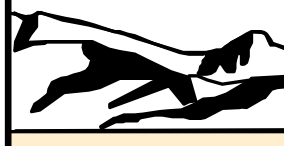

Environmental Restoration

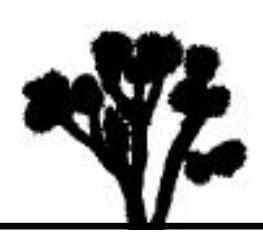

Division 
Available to the public from -
U.S. Department of Commerce
National Technical Information Service
5285 Port Royal Road
Springfield, VA 22161
(703) 487-4650

Available electronically at http://www.doe.gov/bridge. Available to

U.S. Department of Energy and its contractors in paper from -

U.S. Department of Energy

Office of Scientific and Technical Information

P.O. Box 62

Oak Ridge, TN 37831-0062

(423) 576-8401

Reference herein to any specific commercial product, process, or service by trade name, trademark, manufacturer, or otherwise, does not necessarily constitute or imply its endorsement, recommendation, or favoring by the United States Government or any agency thereof or its contractors or subcontractors. 


\title{
CORRECTIVE ACTION DECISION DOCUMENT/ CLOSURE REPORT FOR CORRECTIVE ACTION UNIT 266: AREA 25 BUILDING 3124 LEACHFIELD, NEVADA TEST SITE, NEVADA
}

\author{
DOE Nevada Operations Office \\ Las Vegas, Nevada
}

Controlled Copy No.:

Revision No.: 0

February 2000

Approved for public release; further dissemination unlimited. 


\section{CORRECTIVE ACTION DECISION DOCUMENT/CLOSURE REPORT FOR CORRECTIVE ACTION UNIT 266: \\ AREA 25 BUILDING 3124 LEACHFIELD, NEVADA TEST SITE, NEVADA}

Approved by:

Date: $2 / 17 / 2000$

Janet Appenzeller-Wing, Project Manager

Industrial Sites Project

Approved by:

Signature Approved

Date: $2 / 17 / 2000$

Runore C. Wycoff, Division Director

Environmental Restoration Division 


\section{Table of Contents}

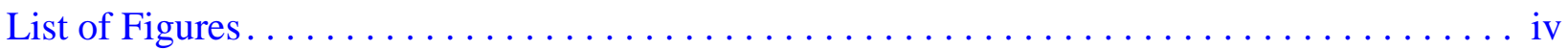

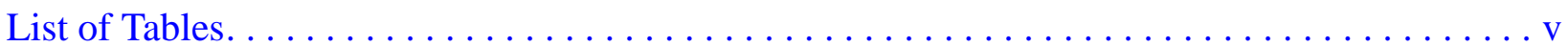

List of Acronyms and Abbreviations $\ldots \ldots \ldots \ldots \ldots \ldots \ldots \ldots \ldots \ldots \ldots \ldots \ldots \ldots \ldots$

Executive Summary . . . . . . . . . . . . . . . . . . . . . . . . .

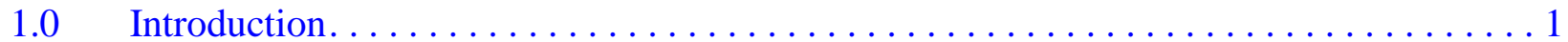

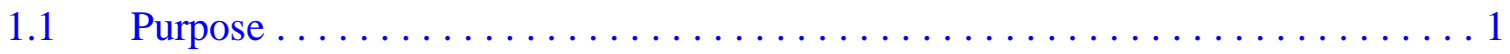

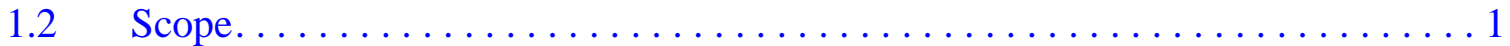

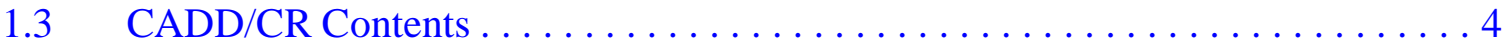

2.0 Corrective Action Investigation Summary $\ldots \ldots \ldots \ldots \ldots \ldots \ldots \ldots \ldots \ldots \ldots \ldots \ldots \ldots$

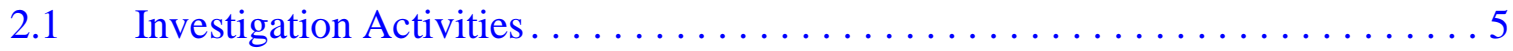

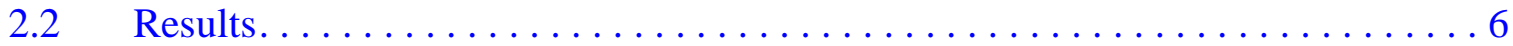

$2.3 \quad$ Need for Corrective Action. . . . . . . . . . . . . . . . . . . . . . 7

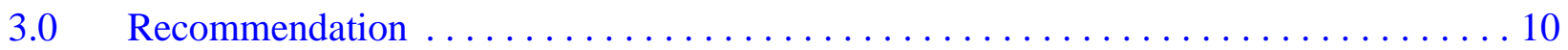

$4.0 \quad$ References................................. 11

Appendix A - Corrective Action Investigation Report for

CAU 266: Area 25 Building 3124 Leachfield, Nevada Test Site, Nevada . . . . . . . . . . . . . . . . . A-1

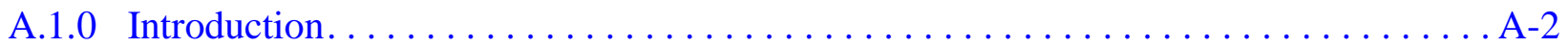

A.1.1 Project Objectives. .......................... A-2

A.1.2 Report Content . . . . . . . . . . . . . . . . . .

A.2.0 Field Investigation and Sampling Activities. . . . . . . . . . . . . . . A-4

A.2.1 Site Descriptions and Conditions . . . . . . . . . . . . . . . . A-5

A.2.2 Septic Tank Sampling. . . . . . . . . . . . . . . . . . . A-5

A.2.3 Video Mole/Radiation Survey . . . . . . . . . . . . . . . . . . A-6

A.2.4 Excavations. . . . . . . . . . . . . . . . . . . . . . . A-6

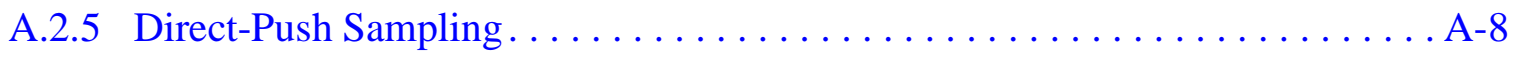

A.2.6 Rotary Sonic Sampling . . . . . . . . . . . . . . . . . . . . A-8

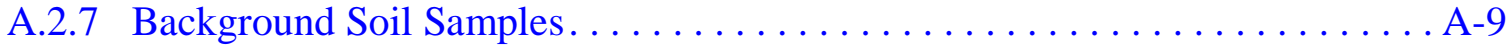

A.2.8 Field Screening . . . . . . . . . . . . . . . . . . . . . . . . A-9

A.2.9 Sample Collection. . . . . . . . . . . . . . . . . . . . . . . A-10

A.2.10 Waste Management. . . . . . . . . . . . . . . . . . . . . A-11

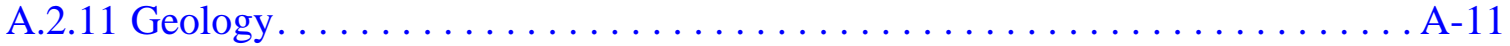

A.2.12 Hydrology . . . . . . . . . . . . . . . . . . . . . . . A 


\section{Table of Contents (Continued)}

A.3.0 Investigation Results. ............................. A-13

A.3.1 Total Volatile Organic Compound Analytical Results ................ . A-17

A.3.2 Total Semivolatile Organic Compound Analytical Results .............. A-18

A.3.3 Total Petroleum Hydrocarbon Results . . . . . . . . . . . . . . . . . . . . . A-19

A.3.4 Total RCRA Metals Results . . . . . . . . . . . . . . . . . . . . . . . A-19

A.3.5 PCB Analytical Results ................................ A-19

A.3.6 Gamma Spectrometry Results $\ldots \ldots \ldots \ldots \ldots \ldots \ldots \ldots \ldots \ldots \ldots$ A -19

A.3.7 Isotope Uranium, Isotopic Plutonium, Isotopic Americium, and Strontium-90 Results ........................... A-23

A.3.8 Septic Tank and Distribution Box Sampling Results ................ A-23

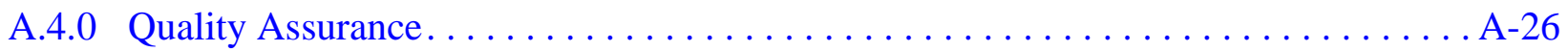

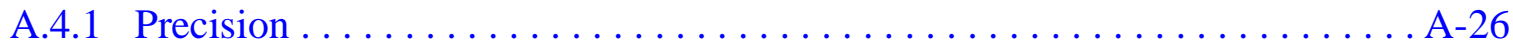

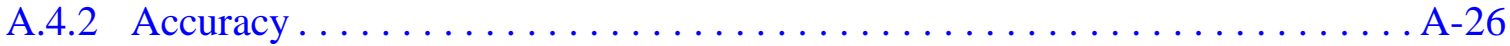

A.4.3 Representativeness . . . . . . . . . . . . . . . . . . . . . . . . A-27

A.4.4 Completeness ................................... A-27

A.4.5 Comparability ................................ 27

A.4.6 Tier I and Tier II Data Evaluations.......................... 28

A.4.6.1 Tier I Evaluation. . . . . . . . . . . . . . . . . . . . A-28

A.4.6.2 Tier II Evaluation . . . . . . . . . . . . . . . . . . . A-29

A.4.6.3 Tier III ................................... A-

A.4.7 Quality Control Samples............................. .

A.4.7.1 Field Quality Control Samples.................... A-31

A.4.7.2 Laboratory Quality Control Samples ............... A-32

A.4.8 Field Nonconformances . . . . . . . . . . . . . . . . . . . . . . . . A-34

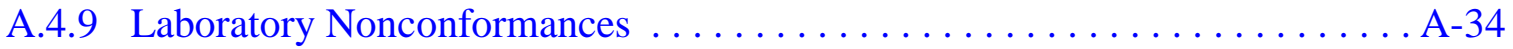

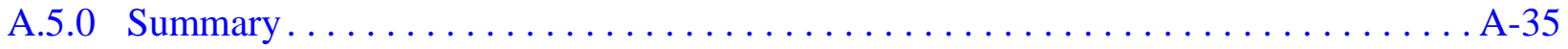

A.6.0 References..................................... A-36

Attachment 1 - Memo from S. Adams to R. McCall entitled, "Dose Assessments of the Americium-241 in Soil at the Area 25 Building 3124 Leachfield.". . . . . . . A-39

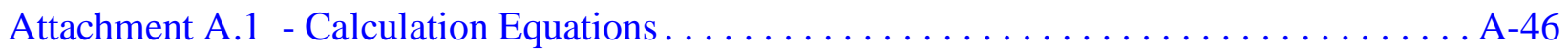

Attachment A.2 - Dose and Risk Assessment Due to Am-241 in Soil at CAU 266 Leachfield.......................... A-52

Appendix B - Radiological Walkover Survey Report ..................... B-1 


\section{Table of Contents (Continued)}

Appendix C - Closure Activities Summary $\ldots \ldots \ldots \ldots \ldots \ldots \ldots \ldots \ldots \ldots \ldots \ldots \ldots$

Appendix D - Waste Generator Approval Process Flow Diagram . . . . . . . . . . . . D-1

Appendix E - Response to NDEP Comments . . . . . . . . . . . . . . . . . E-1 


\section{List of Figures}

Number

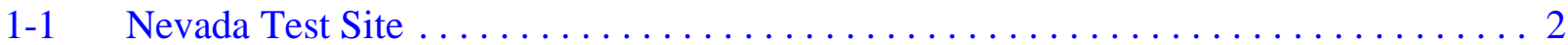

1-2 Location of CAU 266, Area 25, Nevada Test Site, Nevada . . . . . . . . . . . . . 3

A.2-1 CAU 266 Sampling Locations. . . . . . . . . . . . . A

B-1 Test Cell A Background Soil Measurement Locations . . . . . . . . . . . . . . B-4 


\section{List of Tables}

Number

Title

Page

A.3-1 Samples Collected During the CAU 266 Area 25 Building 3124 Leachfield

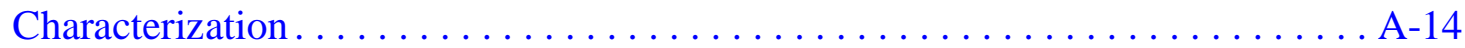

A.3-2 Laboratory Analytical Methods Used for Samples Collected at the CAU 266 Area 25 Building 3124 Leachfield Characterization.

A.3-3 Soil Sample Results for Total Volatile Organic Compounds Detected Above Minimum Reporting Limits, CAU 266 Area 25

Building 3124 Leachfield

A.3-4 Soil Samples for Total RCRA Metals Detected Above Minimum Reporting

Limits Corrective Action Unit 266 Area 25 Building 3124 Leachfield,

Nevada Test Site

A.3-5 Soil Sample Results for PCBs Detected Above Minimum Reporting Limits, CAU 266 Area 25 Building 3124 Leachfield

A.3-6 Soil Sample Results for Gamma-Emitting Radionuclides Above MRLs, CAU 266 Area 25 Building 3124 Leachfield

A.3-7 Soil Sample Results for Isotopic Americium and Isotopic Uranium,

CAU 266 Area 25 Building 3124 Leachfield . .

A.3-8 Summary of Septic Tank and Distribution Box Sampling Results Detected

Above Minimum Detectable Concentrations, CAU 266 Area 25

Building 3124 Leachfield

1 Summary of Dose and Risk from Residual ${ }^{241}$ Am in Soil at CAU 266

2 Radiological Source Terms Used in the Assessments of CAU 266

3 Calculated Committed Effective Dose Equivalent (mrem/yr) and

Risks from Residual 241-Am in CAU 266 Leachfield System Soil . . . . . . . . . A-44

Table A.1 - Numerical Values Used in the CAU 266 Dose and Risk Assessments 


\section{List of Acronyms and Abbreviations}

\begin{tabular}{|c|c|}
\hline bgs & Below ground surface \\
\hline $\mathrm{CADD} / \mathrm{CR}$ & Corrective Action Decision Document/Closure Report \\
\hline CAI & Corrective Action Investigation \\
\hline CAIP & Corrective Action Investigation Plan \\
\hline CAS & Corrective Action Site \\
\hline CAU & Corrective Action Unit \\
\hline CLP & Contract Laboratory Program \\
\hline $\mathrm{cm}^{2}$ & Square centimeter(s) \\
\hline $\mathrm{COC}$ & Contaminant(s) of concern \\
\hline $\mathrm{COPC}$ & Contaminant(s) of potential concern \\
\hline $\mathrm{DOE} / \mathrm{NV}$ & U.S. Department of Energy, Nevada Operations Office \\
\hline dpm & Disintegration(s) per minute \\
\hline DQO & Data Quality Objective(s) \\
\hline EPA & U.S. Environmental Protection Agency \\
\hline FFACO & Federal Facility Agreement and Consent Order \\
\hline FSL & Field-screening level(s) \\
\hline $\mathrm{ft}$ & Foot (feet) \\
\hline GM & Geiger-Muller \\
\hline $\mathrm{ICP}$ & Inductively coupled plasma \\
\hline IDW & Investigation-derived waste \\
\hline in. & $\operatorname{Inch}(\mathrm{es})$ \\
\hline LCS & Laboratory control sample(s) \\
\hline $\mathrm{mi}$ & Mile(s) \\
\hline $\mathrm{mg} / \mathrm{kg}$ & Milligram(s) per kilogram \\
\hline $\mathrm{mg} / \mathrm{L}$ & Milligram(s) per liter \\
\hline $\mathrm{mrem} / \mathrm{yr}$ & Millirem(s) per year \\
\hline MS/MSD & Matrix spike/matrix spike duplicate \\
\hline
\end{tabular}




\section{List of Acronyms and Abbreviations (Continued)}

\begin{tabular}{|c|c|}
\hline NAC & Nevada Administrative Code \\
\hline NDEP & Nevada Division of Environmental Protection \\
\hline NIST & National Institute for Standards and Technology \\
\hline NRDS & Nuclear Rocket Development Station \\
\hline NTS & Nevada Test Site \\
\hline PAL & Preliminary action level(s) \\
\hline PCB & Polychlorinated biphenyls \\
\hline $\mathrm{pCi} / \mathrm{g}$ & Picocurie(s) per gram \\
\hline $\mathrm{PE}$ & Polyethylene \\
\hline PID & Photoionization detector \\
\hline PPE & Personal protective equipment \\
\hline ppm & Part(s) per million \\
\hline PRG & Preliminary Remediation Goals \\
\hline QA & Quality assurance \\
\hline QAPP & Quality Assurance Project Plan \\
\hline QC & Quality control \\
\hline RCRA & Resource Conservation and Recovery Act \\
\hline RPD & Relative percent difference \\
\hline SDG & Sample delivery group \\
\hline SVOC & Semivolatile organic compound(s) \\
\hline TCLP & Toxicity characteristic leaching procedure \\
\hline $\mathrm{TPH}$ & Total petroleum hydrocarbons \\
\hline VOC & Volatile organic compound(s) \\
\hline$\mu \mathrm{g} / \mathrm{kg}$ & Microgram(s) per kilogram \\
\hline$\mu \mathrm{g} / \mathrm{L}$ & Microgram(s) per liter \\
\hline$\mu \mathrm{R} / \mathrm{hr}$ & Microroentgen per hour \\
\hline$\% \mathrm{R}$ & Percent recovery \\
\hline
\end{tabular}




\section{Executive Summary}

This Corrective Action Decision Document/Closure Report has been prepared for Corrective Action Unit 266, Area 25 Building 3124 Leachfield, in accordance with the Federal Facility Agreement and Consent Order (FFACO, 1996). Corrective Action Site 25-05-09 is the only Corrective Action Site within Corrective Action Unit 266. Data collected during the field investigation activities showed evidence of contamination at this site in the soil, septic tank, and distribution box. The Corrective Action Decision Document and Closure Report have been combined into one report because the contaminants of concern were either not present in the soil, or present at concentrations not requiring corrective action. Contaminants identified within the septic tank and distribution box have been adequately addressed.

The purpose of this Corrective Action Decision Document/Closure Report is to justify and recommend that no corrective action is required at Corrective Action Unit 266. To achieve this, the following actions are required:

- Review the current site conditions, including the concentration and extent of contamination.

- Document closure of the Corrective Action Unit.

From February 10 through May 25, 1999, corrective action investigation activities were performed as set forth in the Corrective Action Investigation Plan (DOE/NV, 1999). The purpose of the corrective action investigation is described as follows:

- Identify the presence, distribution, and concentration of contaminants of potential concern at the Corrective Action Unit.

- Determine the vertical and lateral extent of contaminants of potential concern.

- Provide sufficient information and data to develop appropriate corrective actions for the Corrective Action Unit.

Analytes detected during the corrective action investigation were evaluated against preliminary action levels to determine contaminants of concern for Corrective Action Unit 266. Analysis of the data generated from corrective action investigation activities indicates the preliminary action levels were not exceeded for total volatile organic compounds, total semivolatile organic compounds, total petroleum hydrocarbons, polychlorinated biphenyls, total Resource Conservation and Recovery Act 
metals, gamma-emitting radionuclides, isotopic uranium, isotopic plutonium, and strontium-90 for any of the soil samples collected from Corrective Action Unit 266. However, contaminants of concern were identified in two soil samples and within the septic tank and distribution box. The isotopic americium concentrations in two soil samples exceeded preliminary action levels. Because the calculated dose from the residual americium-241 results in the soil is less than the 100 millirems per year limit established by U.S. Department of Energy (DOE) Order 5400.5 (DOE, 1993), no corrective action is necessary for the soil.

Closure activities were performed to address the contaminants of concern identified within the septic tank and distribution box. Based on the results of the closure activities, no further corrective action is necessary for these structures.

The U.S. Department of Energy, Nevada Operations Office provides the following recommendations:

- $\quad$ No corrective action is required at Corrective Action Unit 266.

- No corrective action plan is required.

- A Notice of Completion to the U.S. Department of Energy, Nevada Operations Office, is requested from the Nevada Division of Environmental Protection for the closure of Corrective Action Unit 266, Area 25 Building 3124 Leachfield (Corrective Action Site 25-05-09).

- Corrective Action Unit 266 should be moved from Appendix III to Appendix IV of the Federal Facility Agreement and Consent Order.

No use restrictions are required to be placed on Corrective Action Unit 266 because the investigation revealed soil contamination to be less than the 100 millirems per year limit established by DOE Order 5400.5 (DOE, 1993). The septic tank and distribution box have been closed in accordance with applicable regulations (Nevada Administrative Code 444.818 [1997]). 


\subsection{Introduction}

This Corrective Action Decision Document/Closure Report (CADD/CR) has been prepared for Corrective Action Unit (CAU) 266, Area 25 Building 3124 Leachfield, in accordance with the Federal Facility Agreement and Consent Order (FFACO) that was agreed to by the U.S. Department of Energy, Nevada Operations Office (DOE/NV); the Nevada Division of Environmental Protection (NDEP); and the U.S. Department of Defense (FFACO, 1996). Corrective Action Site (CAS) 25-05-09 is the only CAS within CAU 266. The CADD and CR have been combined into one report because sample data collected during the corrective action investigation indicated that contaminants of concern (COCs) were either not present in the soil, or present at concentrations not requiring corrective action.

Corrective Action Unit 266 is located in Area 25 at the Nevada Test Site (NTS) in Nevada. The NTS is approximately 65 miles (mi) northwest of Las Vegas, Nevada (Figure 1-1 and Figure 1-2).

\subsection{Purpose}

This CADD/CR provides justification for no further action at CAU 266. The justification is based on the results of investigative activities conducted in accordance with the Corrective Action Investigation Plan for Corrective Action Unit 266, Area 25 Building 3124 Leachfield, Nevada Test Site, Nevada (DOE/NV, 1999a) and the Work Plan for Leachfield Corrective Action Units: Nevada Test Site and Tonopah Test Range, Nevada (DOE/NV, 1998).

\subsection{Scope}

The scope of this CADD/CR is to justify and recommend that no corrective action is required at CAU 266. To achieve this scope the following actions are required:

- Review the current site conditions including the concentration and extent of contaminants.

- Document closure of the CAU. 


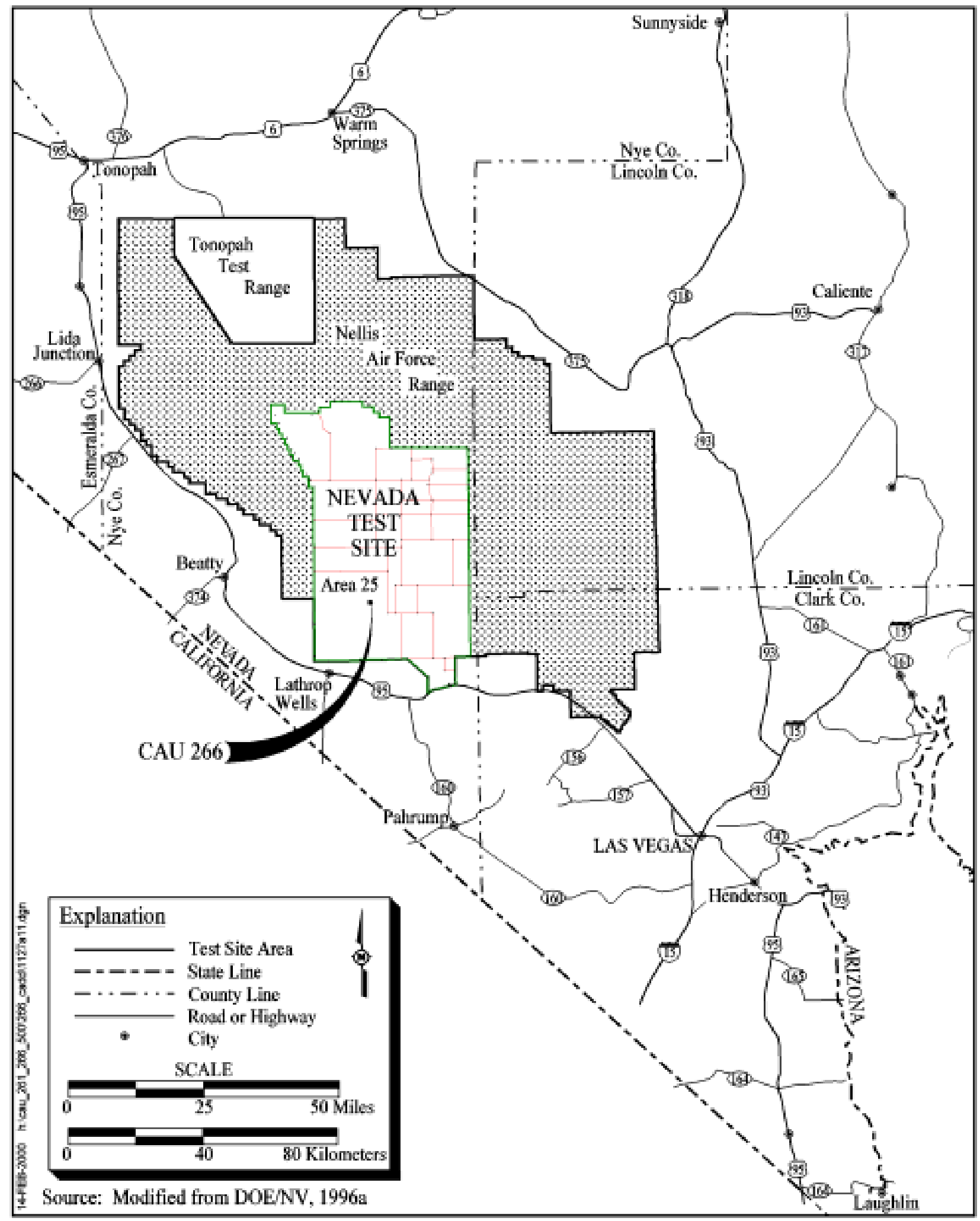

Figure 1-1 


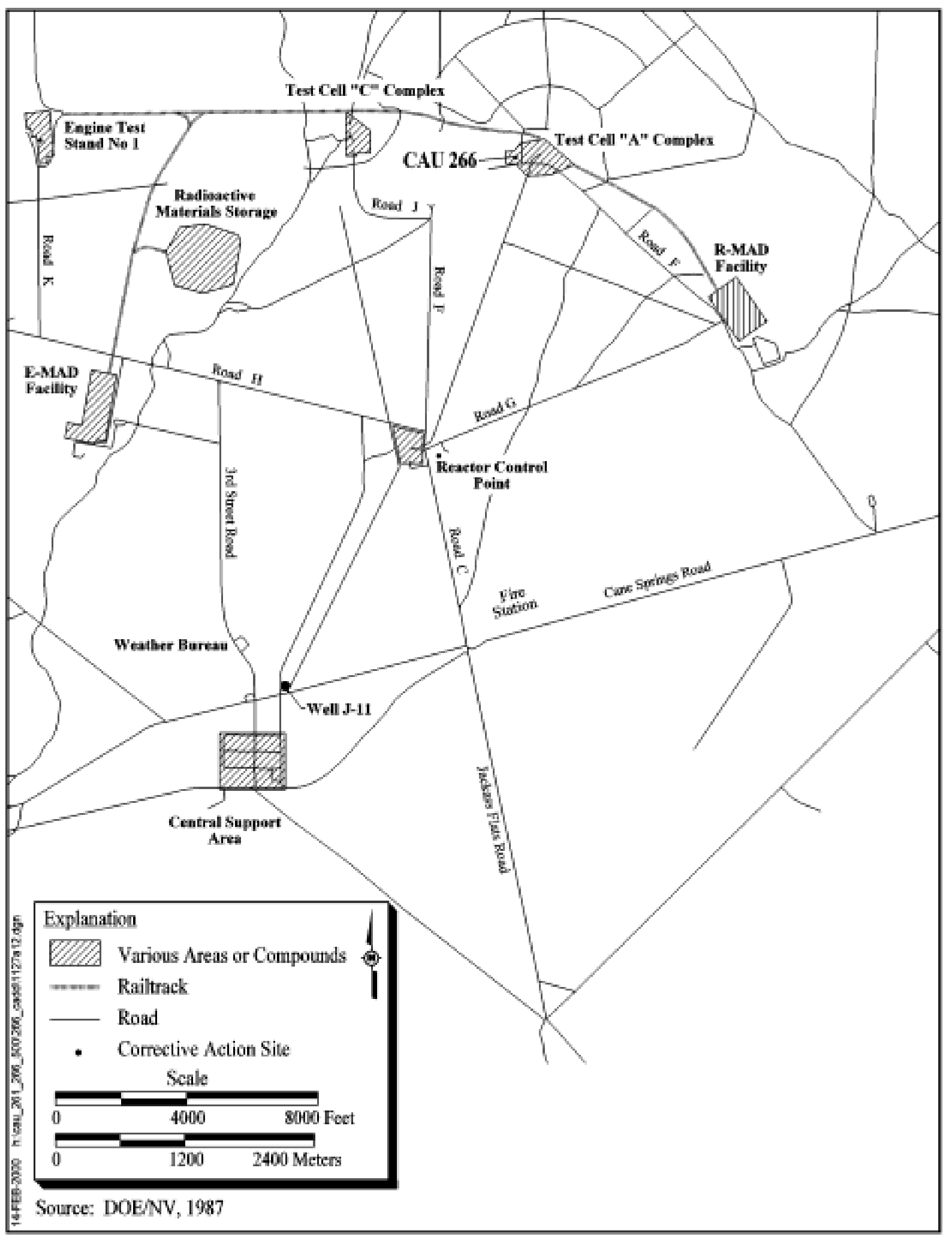




\section{$1.3 \quad C A D D / C R$ Contents}

This CADD/CR is divided into the following sections:

Section 1.0 - Introduction: summarizes the purpose, scope, and contents of this CADD/CR.

Section 2.0 - Corrective Action Investigation Summary: summarizes the investigation field activities, the results of the investigation, and the justification for no further action.

Section 3.0 - Recommendation: recommends no further action is required at the CAU and requests a Notice of Completion.

Section 4.0 - References: provides a list of all referenced documents.

Appendix A - Corrective Action Investigation Report for CAU 266: Building 3124 Leachfield, Nevada Test Site, Nevada.

Appendix B - Radiological Walkover Survey Report

Appendix C - Closure Activities Summary

Appendix D - Waste Generator Approval Process Flow Diagram

Appendix E - Responses to NDEP Comments

All work was performed in accordance with the following documents:

- Corrective Action Investigation Plan for Corrective Action Unit 266, Area 25 Building 3124 Leachfield, Nevada Test Site, Nevada, Rev. 1, DOE/NV--529 (DOE/NV, 1999a)

- Work Plan for Leachfield Corrective Action Units: Nevada Test Site and Tonopah Test Range, Nevada, Rev. 1, DOE/NV--514 (DOE/NV, 1998).

- Industrial Sites Quality Assurance Project Plan, Rev. 1, DOE/NV--372 (DOE/NV, 1996b)

- $\quad$ FFACO (FFACO, 1996)

- Project Management Plan, Rev. 0 (DOE/NV, 1994) 


\subsection{Corrective Action Investigation Summary}

The following sections describe and summarize the results of the investigation activities conducted at CAU 266. For detailed investigation results, please refer to Appendix A.

\section{$2.1 \quad$ Investigation Activities}

From February 10 through May 25, 1999, corrective action investigation activities were performed as set forth in the Corrective Action Investigation Plan (CAIP) (DOE/NV, 1999a). The purpose of the investigation is described as follows:

- Identify the presence and concentrations of contaminants of potential concern (COPCs) at the CAU.

- Determine the vertical and lateral extent of COPCs.

- Provide sufficient information and data to develop appropriate corrective actions for the CAU.

The investigation activities were conducted in three stages. The activities for each stage are summarized below:

\section{$\underline{\text { Stage I }}$}

- The first stage was conducted on February 10 and 11, 1999, and is summarized as follows: sampled the contents of the septic tank and distribution box using hand tools for the collection of liquid and sludge samples. Two sludge and four liquid samples were collected and submitted for laboratory analyses.

- Field screened sludge samples for volatile organic compounds (VOCs) and alpha, beta, and gamma emitters.

- Analyzed sludge and liquid samples for total VOCs; total semivolatile organic compounds (SVOCs); total Resource Conservation and Recovery Act (RCRA) metals; total petroleum hydrocarbons (TPH) as diesel/oil, polychlorinated biphenyls (PCBs), gamma-emitting radionuclides, isotopic uranium, isotopic plutonium, isotopic americium, and strontium-90. Additionally, the sludge samples were analyzed according to the toxicity characteristic leaching procedure (TCLP) for VOCs, SVOCs, and RCRA metals.

- Evaluated sample analytical results from Stage I to guide Stage III. 
$\underline{\text { Stage II }}$

The second stage of the investigation consisted of a video mole and radiation survey of the discharge and outfall piping to identify the collection system and lines leading into the leachfield. The objectives of the video mole survey were to identify tie-ins, outfalls, broken pipes, and radiation in pipes. The second stage occurred from March 22 through 26, 1999, and April 20, 21, and 28, 1999. No sampling was conducted during this stage.

\section{$\underline{\text { Stage III }}$}

The third (main) stage of the investigation consisted of determining the leachfield piping configuration using a backhoe, and soil sampling using a Geoprobe ${ }^{\circledR}$ and a rotary sonic drill rig. This stage occurred from April 28 through May 25, 1999, and is summarized as follows:

- Excavated with a backhoe to identify the leachfield piping configuration and to stake sampling locations.

- Drilled 15 boreholes within the leachfield to collect soil samples below the leachrock for laboratory analyses.

- Conducted field screening for radiological constituents and VOCs. Conducted visual field screening and logged soil cuttings to assess the site geology.

- Collected soil samples for bioassessment and geotechnical analysis from below the base of the leachfield.

- Analyzed soil samples for VOCs, SVOCs, RCRA metals, TPH-diesel/oil, and PCBs. More than 25 percent of the samples submitted to the laboratory were also analyzed for gamma-emitting radionuclides, isotopic uranium, isotopic plutonium, isotopic americium, and strontium-90.

\subsection{Results}

Analysis of the data generated from corrective action investigation activities conducted at CAU 266, Area 25 Building 3124 Leachfield, indicates the following:

- The preliminary action levels (PALs) were not exceeded in soil samples for total VOCs, total SVOCs, TPH, total PCBs, total RCRA metals, gamma-emitting radionuclides, isotopic uranium, isotopic plutonium, and strontium- 90 for any of the samples collected from the site. 
- The isotopic americium (americium-241) concentrations in two soil samples exceed PALs; therefore, americium-241 is a COC at CAU 266. The calculated dose from the residual americium-241 in the soil results is less than the 100 millirems per year (mrem/yr) limit established by U.S. Department of Energy Order 5400.5 (DOE, 1993).

- Analytical results from liquid samples collected from the septic tank indicated detection of COPCs to include 1,4-dichlorobenzene, p-isopropyltoluene, trichloroethene, bis(2-ethylhexyl)phthalate, americium-241, uranium-234, and uranium-238. There were no toxicity characteristic chemical COPCs detected above the regulatory levels. Uranium isotopes were detected in ratios that were determined to constitute "added radioactivity."

- Analytical results from sludge samples collected from the septic tank and distribution box indicated detection of COPCs to include americium-241, americium-243, cesium-137, uranium-234, uranium-238, and potassium-40. Americium-241 was detected at levels which were determined to constitute "added radioactivity." Aroclor-1260 was detected at 2,200 micrograms per kilogram $(\mu \mathrm{g} / \mathrm{kg})$ which is below the regulatory level of 50 milligrams per kilogram $(\mathrm{mg} / \mathrm{kg})$. Diesel-range organics were detected in the septic tank sludge at $1,400 \mathrm{mg} / \mathrm{kg}$, which exceeds the Nevada regulatory level of $100 \mathrm{mg} / \mathrm{kg}$, and in the distribution box at $78 \mathrm{mg} / \mathrm{kg}$.

- Analytical results from sludge samples collected from the septic tank and distribution box and processed using the TCLP method indicated detection of COPCs to include barium, cadmium, and lead. None of these COPCs were detected above the toxicity characteristic regulatory levels.

- The geologic conditions revealed that the soil within the leachfield is comprised of alluvial fan soil that is poorly sorted with abundant gravel and cobbles. Thin lenses of sand and silt were present at some locations. The soil beneath the leachrock was a poorly sorted, silty gravelly sand with abundant pebbles, some gravel, and a few cobbles.

- The geotechnical and bioassessment samples will not be submitted for analysis because the results will not significantly benefit the corrective action decision process.

Details of the methods used and results found during the investigation are presented in Appendix A. Based on these results, the CAU 266 site has been adequately characterized.

\subsection{Need for Corrective Action}

Analytes detected during the corrective action investigation were evaluated against PALs to determine COCs for CAU 266. Contaminants of concern were identified in two soil samples and within the septic tank and distribution box. Because the calculated dose from the residual americium-241 results in the soil is less than the $100 \mathrm{mrem} / \mathrm{yr}$ limit established by DOE Order 5400.5 
(DOE, 1993), no corrective action is necessary for the soil. Information supporting the dose assessment calculations is contained in Attachment 1 of Appendix A. The septic tank and distribution box have been closed in accordance with applicable regulations (Nevada Administrative Code [NAC] 444.818 [1997]).

Septic tank and distribution box closure activities were performed by Bechtel Nevada from December 13, 1999, through January 20, 2000 (Cowser, 2000). The activities included the following:

- $\quad$ Removed contents of the septic tank.

- Pressure-washed the septic tank and distribution box interiors.

- Solidified the septic tank contents and associated rinsate from the septic tank and distribution box for disposal.

- Collected verification samples from the septic tank rinsate.

- Grouted the septic tank and distribution box in place including the influent and effluent lines near the septic tank.

The rinsate from the septic tank was analyzed for TPH and gross alpha and gross beta. The established closure criteria for TPH is the NAC Action Level of 100 parts per million (ppm) using U.S. Environmental Protection Agency's (EPA's) Method 8015 (modified). The established closure criteria for radiological constituents is the EPA Drinking Water Standards of 15 picocuries per liter (pCi/L) for gross alpha and $50 \mathrm{pCi} / \mathrm{L}$ for gross beta.

Analytical results of the final rinsate from the septic tank met closure criteria. Results indicated TPH as gasoline less than 0.25 milligrams per liter $(\mathrm{mg} / \mathrm{L})$ and $\mathrm{TPH}$ as diesel at $0.5 \mathrm{mg} / \mathrm{L}$. Gross alpha was detected at $4.61 \mathrm{pCi} / \mathrm{L}$ and gross beta at $11.1 \mathrm{pCi} / \mathrm{L}$.

Rinsate samples from the distribution box were inadvertently omitted and verification that the closure criteria was met is unattainable. However, site conditions indicate that the distribution box was sufficiently cleaned and does not pose a health or environmental risk based on the following:

- The interior of the distribution box was pressure washed.

- Minimal residual waste was present in the distribution box prior to pressure washing. 
- Concentrations of radioisotopes and petroleum hydrocarbons detected in the original characterization sample analysis were very low. The characterization data is available in Appendix A.

- Identical procedure used to decontaminate the septic tank in which contaminant concentrations were much higher than those of the distribution box

- The box is structurally intact indicating that nothing has been or will be released into the underlying soil.

- The box was sealed to the surface with grout.

Refer to Appendix A (Cowser, 2000) for a more detailed description of closure activities and rinsate results.

The waste profile has been approved by the Radioactive Waste Acceptance Program. See Appendix D for a description of the NTS Waste Acceptance process (DOE/NV, 1999b). The waste is expected to be disposed of by February 29, 2000. Waste manifests and disposal documentation will be available subsequent to disposal. 


\subsection{Recommendation}

Based on the results of the corrective action investigation in Appendix A, a COC has been identified in the soil at CAU 266. However, the concentrations pose an acceptable risk to human health and the environment. Results from the closure activities associated with the septic tank and distribution box presented in Appendix $\mathrm{C}$ indicate that no further corrective action is necessary for these structures. The DOE/NV provides the following recommendations based on the results of the corrective action investigation:

- No corrective action is required at CAU 266.

- No corrective action plan is required.

- A Notice of Completion to DOE/NV is requested from NDEP for the closure of CAU 266, Area 25 Building 3124 Leachfield (CAS 25-05-09).

- CAU 266 should be moved from Appendix III to Appendix IV of the FFACO.

No use restrictions are required to be placed on CAU 266 because the investigation revealed soil contamination to be less than the $100 \mathrm{mrem} / \mathrm{yr}$ limit established by DOE Order 5400.5 (1993). The septic tank and distribution box have been closed in accordance with applicable regulations NAC 444.818 [1997]). 


\subsection{References}

Cowser, D.K. 2000. Memorandum to J.L. Appenzeller-Wing (DOE/NV) regarding the Summary Closure for Corrective Action Units (CAUs) 266 and 500, Area 25 Test Cell A Septic Systems, Nevada Test Site, NV, 27 January. Las Vegas, NV: Bechtel Nevada.

DOE/NV, see U.S. Department of Energy, Nevada Operations Office.

FFACO, see Federal Facility Agreement and Consent Order.

Federal Facility Agreement and Consent Order. 1996 (as amended). Agreed to by the State of Nevada, the U.S. Department of Energy, and the U.S. Department of Defense.

NAC, see Nevada Administrative Code.

Nevada Administrative Code. 1997. NAC 444, "Sanitation." Carson City, NV.

U.S. Department of Energy. 1993. Radiation Protection of the Public and the Environment, DOE Order 5400.5. Washington, DC.

U.S. Department of Energy, Nevada Operations Office. 1994. Project Management Plan, Rev. 0. Las Vegas, NV.

U.S. Department of Energy, Nevada Operations Office. 1987. Nevada Test Site - Environmental Compliance Atlas, 15 June. Las Vegas, NV.

U.S. Department of Energy, Nevada Operations Office. 1996a. Final Environmental Impact Statement for the Nevada Test Site and Off-Site Locations in the State of Nevada, DOE/EIS 0243. Las Vegas, NV.

U.S. Department of Energy, Nevada Operations Office. 1996b. Industrial Sites Quality Assurance Project Plan, Nevada Test Site, Nevada, Rev. 1, DOE/NV--372. Las Vegas, NV.

U.S. Department of Energy, Nevada Operations Office. 1998. Work Plan for Leachfield Corrective Action Units: Nevada Test Site and Tonopah Test Range, Nevada, Rev. 1, DOE/NV--514. Las Vegas, NV.

U.S. Department of Energy, Nevada Operations Office. 1999a. Corrective Action Investigation Plan for Corrective Action Unit 266: Area 25 Building 3124 Leachfield, Nevada Test Site, Nevada, Rev. 1, DOE/NV--529. Las Vegas, NV.

U.S. Department of Energy, Nevada Operations Office. 1999b. Nevada Test Site Waste Acceptance Criteria, Rev. 2, DOE/NV--325. Las Vegas, NV. 


\section{Appendix A}

\section{Corrective Action Investigation Report for CAU 266: Area 25 Building 3124 Leachfield, Nevada Test Site, Nevada}




\section{A.1.0 Introduction}

This appendix presents corrective action investigation (CAI) activities and analytical results for CAU 266, Area 25 Building 3124 Leachfield at the NTS. This CAU includes CAS 25-05-09, Leachfield (FFACO, 1996). The corrective action investigation was conducted in accordance with the Corrective Action Investigation Plan for Corrective Action Unit 266: Area 25 Building 3124 Leachfield, Nevada Test Site, Nevada (DOE/NV, 1999) as developed under the FFACO (1996).

Test Cell A operated during the 1960s and 1970s to support nuclear rocket reactor testing as part of the Nuclear Rocket Development Station (NRDS) (SNPO, 1970). Various operations within Building 3124, from 1962 through 1995, have resulted in liquid waste releases to the leachfield (DOE/NV, 1999). This CAU was investigated because process knowledge indicated that the subsurface soils in the vicinity of the collection system and leachfield may have been impacted by radioactive and other COPCs associated with activities from Building 3124. Additional information regarding the history of the site, planning, and the scope of the investigation is presented in the CAIP (DOE/NV, 1999) and will not be repeated in this report.

\section{A.1.1 Project Objectives}

The primary objectives of the investigation were as follows:

- Identify the presence and the vertical and lateral extent of COPCs.

- Provide sufficient information and data to develop appropriate corrective action alternatives.

The selection of soil sample locations for the site was based on site conditions and the strategy developed during the data quality objectives (DQO) process as outlined in the CAIP (DOE/NV, 1999).

\section{A.1.2 Report Content}

This report contains information and data in sufficient detail to support the selection of no further action in the CADD/CR. The contents of this report are as follows:

- Section A.1.0 describes the investigation background, objectives, and the report content. 
- Section A.2.0 provides information regarding the field activities and sampling methods.

- Section A.3.0 summarizes the results of the laboratory analyses from the investigation sampling.

- Section A.4.0 discusses the quality assurance (QA) and quality control (QC) procedures that were followed and the results of the QA/QC activities.

- Section A.5.0 is a summary of the investigation results.

- Section A.6.0 provides the cited references.

The complete field documentation and laboratory data, including Field Activity Daily Logs, Sample Collection Logs, Analyses Request/Chain-of-Custody Forms, soil sample descriptions, laboratory certificates of analyses, analytical results, and surveillance results are retained in project files. 


\section{A.2.0 Field Investigation and Sampling Activities}

The following is a brief summary of all CAI activities provided as background information:

- Conducted surface radiation survey to determine surface radioactivity.

- Collected samples from the septic tank and distribution box for laboratory analyses.

- Performed video mole and radiation surveys of portions of the collection system.

- Excavated with a backhoe to identify the leachfield piping configuration.

- Drilled 15 boreholes within the leachfield.

- Conducted field screening for radiological activity and VOCs

- Conducted visual field screening

- Collected environmental samples for laboratory analyses

- Logged soil cuttings to assess site geology

- Collected soil samples for bioassessment and geotechnical analyses using a direct-push method $\left(\right.$ Geoprobe $\left.^{\circledR}\right)$.

The field investigation was conducted in three separate stages. A radiological walkover survey was conducted prior to sampling activities and is not included as part of the three stages. The radiological walkover survey results are included in Appendix B. The first stage, conducted on February 10 and 11, 1999, consisted of collecting liquid and sludge samples from the septic tank and distribution box using hand tools.

The second stage of the investigation consisted of a video mole and radiation survey of the discharge and outfall piping to identify the collection system and lines leading into the leachfield. The objectives of the video mole survey were to identify tie-ins, outfalls, broken pipes, and radiation in the pipes. The second stage occurred from March 22 through 26, 1999, and April 20, 21, and 28, 1999. No sampling was conducted during this stage. 
The third (main) stage of the investigation consisted of determining the leachfield piping configuration using a backhoe, and soil sampling of potentially affected areas using a rotary sonic drill rig. During the field investigation, soil removed from excavations and sampling locations was returned nearest to its original location. This stage occurred from April 28 through May 25, 1999.

The field investigation and sampling program was managed in accordance with the requirements set forth in the CAIP (DOE/NV, 1999) and the Work Plan for Leachfield CAUs (DOE/NV, 1998). The field activities were performed in accordance with an approved Site-Specific Health and Safety Plan (IT, 1999). The samples were collected and documented by following approved sampling procedures. Quality control samples (e.g., field blanks, equipment rinsate blanks, trip blanks, and sample duplicates) were collected as required by the Industrial Sites Quality Assurance Project Plan (QAPP) (DOE/NV, 1996b) and approved procedures. During field activities, waste minimization practices were followed according to approved procedures, including segregation of the waste by waste stream.

\section{A.2.1 Site Descriptions and Conditions}

Corrective Action Unit 266 is located at Test Cell A in Area 25 of the NTS (see Figure 1-2 of the CADD/CR). The site is comprised of the leachfield, septic tank, distribution box, and associated piping. The leachfield is located southwest of Building 3124, which is southwest and adjacent to Test Cell A.

During the investigation, the weather conditions at the site were generally favorable and varied from sunny and hot to rain showers and some snow. Sampling activities were halted on occasion, due to weather conditions.

\section{A.2.2 Septic Tank Sampling}

First stage sampling was conducted at the septic tank and distribution box located adjacent to the leachfield. Liquid and sludge samples were collected from within the septic tank and a sludge sample was collected from the distribution box. These samples were collected by hand using long-handle scoops. The samples collected from the septic tank and distribution box were submitted for analyses and the results were used to help guide the third stage of the investigation. The liquid and sludge 
samples were analyzed for total VOCs, SVOCs, RCRA metals, TPH-diesel/oil, PCBs, gammaemitting radionuclides, isotopic uranium, isotopic plutonium, isotopic americium, and strontium-90. Additionally, the sludge samples (TCA60002 and TCA60003) were processed using the TCLP method prior to analyzing for total VOCs, SVOCs, and RCRA metals.

\section{A.2.3 Video Mole/Radiation Survey}

The second stage of the investigation involved use of the video mole. The video mole was used to investigate the integrity of the piping, identify tie-ins, and obtain gross gamma activity within the pipes. The video mole was introduced into the pipes and a Geiger-Muller (GM) detector was attached behind the camera head. No elevated radiation (greater than 100 microroentgen per hour $[\mu \mathrm{R} / \mathrm{hr}]$ ) was observed in any of the pipes that were surveyed with the video mole and the GM detector. The GM detector readings ranged from 26 to $60 \mu \mathrm{R} / \mathrm{hr}$ in the pipes surveyed. No elevated radiation (using the Electra) or volatiles (using a photoionization detector [PID]) above field-screening levels (FSLs) were detected within the pipes near the openings.

One 15-foot (ft) section of pipe was not surveyed due to limited pushing capabilities. This occurred at the pipe between the manhole (outside Building 3124) and the septic tank at the leachfield. No unexpected tie-in or breeches in the pipe were observed. No breeches in the pipe were observed that would warrant additional sampling beyond those locations already planned for this CAU. A videotape was used to record the camera images during the video mole survey. This tape is retained in the project files.

\section{A.2.4 Excavations}

Excavations were used to identify the leachfield piping configuration. The leachfield piping configuration was as expected, and the excavations were used to verify the pipe layout and to locate and stake the sampling locations. Figure A.2-1 shows the leachfield piping configuration and the sampling locations. Soil from the excavation was placed on plastic sheeting or field screened prior to staging on the surface. Field-screening levels were not exceeded for excavated soil. All excavations were backfilled with spoils. Sampling was not conducted during these excavations. 


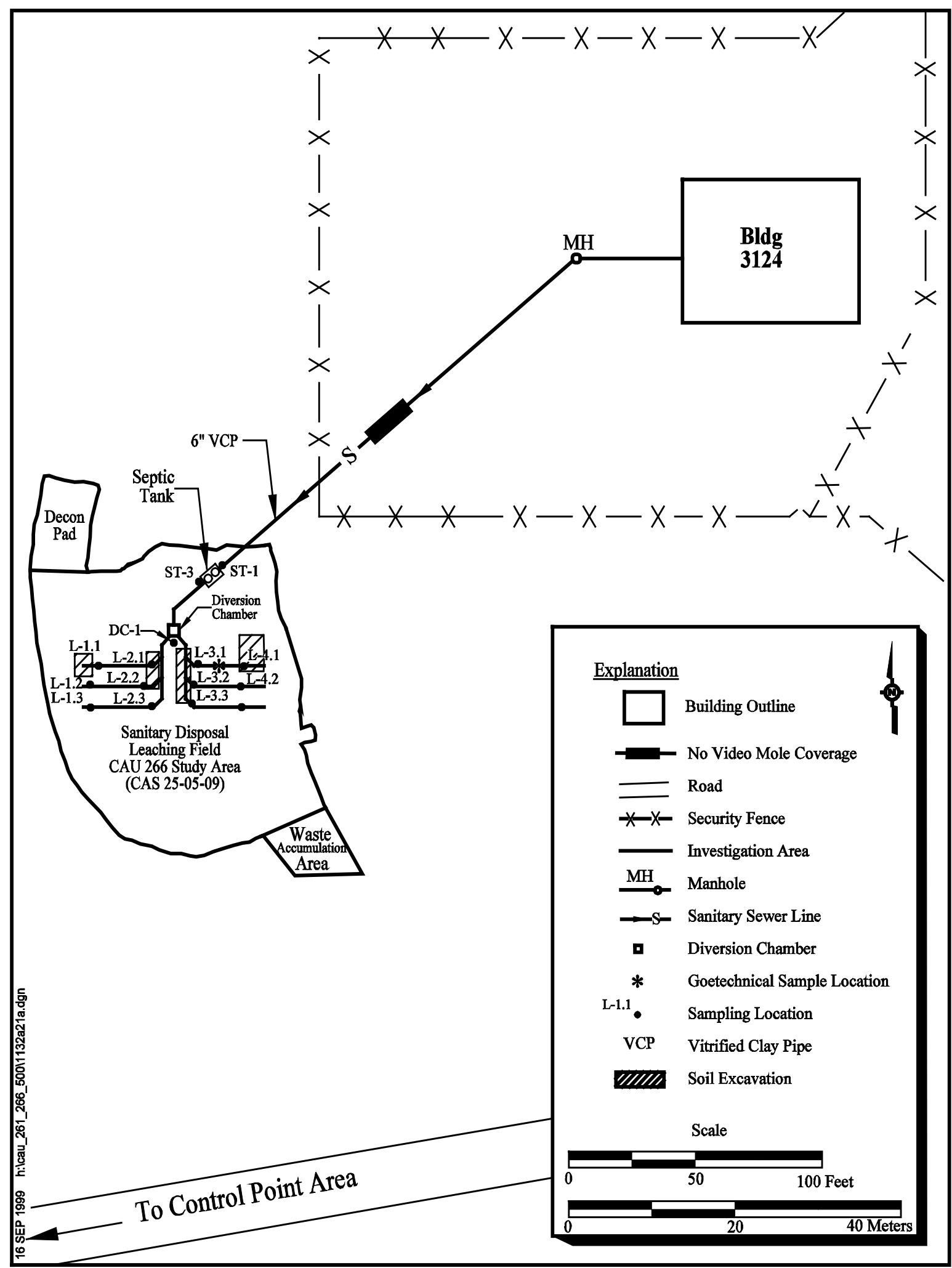

Figure A.2-1 


\section{A.2.5 Direct-Push Sampling}

A direct-push method (Geoprobe ${ }^{\circledR}$ ) was used to collect samples during the third stage of the investigation. Geotechnical and bioassessment soil samples were collected using a Macrocore ${ }^{\circledR}$ sampler with stainless steel liners. The Geoprobe ${ }^{\circledR}$ unit was utilized to collect samples from one location in the native soil beneath the leachfield (see Figure A.2-1). Four 1-ft long by 1.5-inch (in.) diameter samples were collected and placed in a plastic covering with end caps. Due to limited volume constraints within the specified interval, multiple pushes were necessary to obtain adequate soil for the bioassessment and geotechnical analyses.

\section{A.2.6 Rotary Sonic Sampling}

The third stage of the investigation also used rotary sonic drilling, which uses vibration and rotation of the drill string to advance a core barrel. An outer casing was used as needed to stabilize the hole and minimize potential cross contamination produced by soil from shallower levels falling down the hole (sloughing) as the core barrel is removed for cuttings extrusion. After the bit at the end of the core barrel reaches the specified depth, the core barrel is withdrawn from the borehole and the contents are extruded into polyethylene (PE) bags.

A 10-ft long, 6-in. diameter core barrel was advanced. Due to the length of the core barrel and the shallow sampling depths, all of the boreholes were completed without the need for the 8-in. diameter casing, except at L-2.3 where a further advancement (12.5 ft) was necessary. All boreholes were backfilled with unused cuttings.

Sonic drilling was used to sample the soil from below the leachfield. The planned sample locations are shown in Figure 4-2 of the CAIP (DOE/NV, 1999). The leachfield piping configuration and the sampling locations were as expected. The sampling locations were selected based on visual observation and process knowledge. The locations of the 15 boreholes drilled within the leachfield are shown on Figure A.2-1. The soil cuttings were suitable for field screening, sampling, and visual classification of the soil as described in subsequent subsections. No problems were encountered while drilling within the leachfield. Leachrock was encountered within the leachfield ranging in thickness from 1.5 to $2.5 \mathrm{ft}$, and at a depth that ranged from 1.5 to $3.0 \mathrm{ft}$ below ground surface (bgs). 
Soil cuttings were delivered to the field geologist and sampling team in labeled PE bags in 2- to 4-ft long sections. Soil samples were collected from 0 to $1 \mathrm{ft}$ and 2.5 to $3.5 \mathrm{ft}$ below the leachfield base (leachrock/soil interface). These samples were submitted for laboratory analyses. At boring L-2.3, soil samples were submitted to the laboratory from 0 to $1 \mathrm{ft}, 2.5$ to $3.5 \mathrm{ft}$, and 7.5 to $8.5 \mathrm{ft}$ (due to inadequate soil volume) below the leachfield base. Soil samples were submitted to the laboratory from 5 to $6 \mathrm{ft}$ bgs at the influent and effluent ends of the septic tank, below the influent and effluent pipes. Soil samples were submitted to the laboratory from 5 to $6 \mathrm{ft}$ bgs at the effluent end of the distribution box as specified in the CAIP (DOE/NV, 1999). Samples were analyzed for VOCs, SVOCs, RCRA metals, TPH-diesel/oil, and PCBs. A minimum of 25 percent of the samples (including duplicates and matrix spike/matrix spike duplicate [MS/MSD]) submitted to the laboratory were also analyzed for gamma-emitting radionuclides, isotopic uranium, isotopic plutonium, isotopic americium, and strontium-90 based on field-screening results and location. For a complete list of analyses, see Section 3.2 of the CAIP (DOE/NV, 1999). Field screening identified no contamination above FSLs in any of the borings drilled within the leachfield; therefore, no step-out borings were necessary. Only one deeper sampling horizon (at boring L-2.3) was sent to the laboratory because adequate volume was not obtained at the normal sampling depth.

\section{A.2.7 Background Soil Samples}

Six background soil samples were collected by hand from the surface area near Test Cell A (see Figure 1 in Appendix B). The samples were collected from the surface to a depth of $0.5 \mathrm{ft}$ and were analyzed for RCRA metals, gamma-emitting radionuclides, isotopic uranium, isotopic plutonium, isotopic americium, and strontium-90.

\section{A.2.8 Field Screening}

In general, two consecutive "clean" samples, as measured by field-screening methods, were collected to define the lower or lateral limits of the impacted soils. Field screening and surveys were performed as specified in the CAIP (DOE/NV, 1999). The screening and survey methods were as follows:

- Radiological survey for alpha and beta emitters using an Electra instrument. 
- Radiological survey for gamma emitters using an Eberline ESP-2 instrument with a sodium iodide probe.

- Headspace screening for VOCs using a PID.

Field-screening levels were used to guide sample collection both laterally and vertically, and to provide a basis for the collection of additional environmental samples. The FSL for VOCs was $20 \mathrm{ppm}$ or 2.5 times background, whichever was higher. The FSLs for radiation surveying results were established as the average activity of 20 background samples plus two times the standard deviation of the average activity of the 20 background samples (see Figure 1 in Appendix B). The FSL for alpha was established at 110 disintegrations per minute $(\mathrm{dpm}) / 100$ square centimeters $\left(\mathrm{cm}^{2}\right)$ and $2,045 \mathrm{dpm} / 100 \mathrm{~cm}^{2}$ for beta. The FSL for gamma radiation was established at 62,451 counts per minute.

Field screening was performed on all soil samples. For excavations, soil from the initial bucket and every third thereafter was field screened to determine if the spoils had to be staged on plastic sheeting. Some excavated soil was placed on plastic as a precaution; however, the soil did not exceed FSLs.

\section{A.2.9 Sample Collection}

Sample collection was performed as specified in the CAIP (DOE/NV, 1999). During rotary drilling, soil cores were moved from the boreholes to the sampling area in PE bags cut into convenient lengths. The bags were opened and screened for alpha, beta, and gamma radiological contamination. The breathing zone was monitored for VOCs using a PID before and during sample collection. Samples were collected in appropriate containers, temporarily marked with sample label information, sealed with custody tape, and placed in an iced cooler with a trip blank (if applicable). Volatile samples (VOCs, and headspace field screening) were collected directly from the soil cores immediately after required radiation field screening and breathing zone monitoring was conducted. The remainder of the samples were collected from soil representative of the sampling interval after homogenization in a stainless steel bowl.

After samples were identified as laboratory samples, labels with the sample number, sample collection date/time, sampling team members, container preservative, medium type, and requested analyses were attached to each of the containers. Each sample container was then wrapped in 
protective bubble wrap (if applicable), placed into a sealable bag, and stored in an iced cooler with a trip blank (if applicable). Sample media not submitted to the laboratory was returned with the soil cuttings to the collection location. Soil descriptions were recorded on a Sample Collection Log and are retained in project files.

\section{A.2.10 Waste Management}

Investigation-derived waste (IDW) was segregated into the following three waste streams:

- Personal protective equipment (PPE) and disposable sampling equipment that contacted potentially contaminated media

- Decontamination rinsate that contacted potentially contaminated media

- Plastic and minor amounts of soil from the decontamination pad

Soil incidental to sample collection (e.g., soil cuttings, discarded sample media) was placed back into the associated borehole. Potentially hazardous waste generated during site operations was labeled as such and transferred to a Hazardous Waste Accumulation Area daily. The IDW was documented using a waste tracking log. All IDW associated with the collection of samples from the septic tank and distribution box will be managed and disposed of as low-level waste. All other IDW will be disposed of as sanitary waste.

\section{A.2.11 Geology}

Corrective Action Unit 266 is located in Jackass Flats. The Jackass Flats basin was formed by faulting of Paleozoic carbonate rocks. The Paleozoic rock and clastic sediment are approximately 22,000 ft thick and are overlain by welded and semiwelded ash flow and ash fall tuffs of Tertiary age, approximately 5,000 ft thick. The most prominent structural feature in Jackass Flats is a fault which trends northeast and is located west of Well J-11. Surface geology and soils in Area 25 consist of silty sand, ranging from fine sand to coarse sand and gravel. These types of soils are generally unstable and cohesionless. Other rock types in the surrounding area include shales, quartzites, and carbonates of Lower to Middle Cambrian age; carbonate and thin shale layers of Middle Cambrian to Devonian age; and argillites, cherty limestones, and conglomerates of Devonian to Permian age. Soils in the area range from poorly sorted silt to coarse sand and gravel (SNPO, 1970). 
The alluvial fan soil at the Test Cell A site is poorly-sorted with abundant gravel and cobbles. Thin lenses of sand and silt were present at some locations. The alluvial fan soil beneath the leachrock was similar to CAUs 261 and 500 and is a poorly sorted, silty-gravelly sand with abundant pebbles, some gravel, and a few cobbles. No welded tuff layer was encountered at the CAU 266 leachfield. Field descriptions were performed by the sampling team at each borehole location and recorded on Visual Classification of Soil Logs which are maintained in the project files.

\section{A.2.12 Hydrology}

Groundwater at Test Cell A is not expected to be impacted by COPC migration due to the depth to groundwater. Information on depth to groundwater in this vicinity can be found in the Yucca Mountain Site Characterization Plan (DOE, 1988).

Depth to groundwater at the nearest well (J-11) is approximately 1,040 ft bgs and is located 17,200 ft southwest of Test Cell A (USGS, 1993). The distance to the nearest active water-supply well (J-12), is approximately $8.5 \mathrm{mi}$ southwest of Test Cell A (DOE/NV, 1996a). Groundwater flow is generally to the southwest and may discharge at Ash Meadows (Laczniak et al., 1996). There are no perennial surface water sources that would impact CAU 266. However, the site could be potentially impacted by ephemeral drainage due to localized flooding. 


\section{A.3.0 Investigation Results}

The analytical results of samples collected from the CAU 266 investigation have been compiled and evaluated to determine the presence and/or extent of contamination. The analytical results above the minimum reporting limits are summarized in the following subsections.

During the Stage I and Stage III investigation activities (Stage II activities did not include sample collection), 57 samples were submitted to Paragon Analytical Services, Fort Collins, Colorado, for laboratory analysis. During Stage I (septic tank and distribution box sampling), radiological analyses were performed by the Bechtel Nevada Analytical Services Laboratory in Mercury, Nevada, with the exception of the isotopic americium and uranium analyses. These analyses were performed by Paragon Analytical Services. The analytical results of the septic tank and distribution box sampling are discussed separately in Section A.3.8.

A list of the sample numbers from Stages I and III (including quality control samples) and their relationship to the sample locations is presented in Table A.3-1. One sample was collected for geotechnical analysis and one sample for bioassessment analysis. These samples will not be submitted for analysis because the results will not significantly benefit corrective action. A Tier III review of at least five percent of the sample analytical data will be performed by Laboratory Data Consultants, Inc. in Carlsbad, California. The analytical parameters and laboratory analytical methods requested for this investigation are presented in Table A.3-2.

The analytical parameters were selected through the application of site process knowledge according to the EPA's Guidance for the Data Quality Objectives Process (EPA, 1994c). Preliminary action levels for off-site laboratory analytical methods were determined during the DQO process and are documented in the Work Plan for Leachfield CAUs (DOE/NV, 1998). Sampling activities were conducted to confirm or disprove assumptions (i.e., models outlined in CAIP) made in the DQO process (DOE/NV, 1999). 
Table A.3-1

Samples Collected During the CAU 266 Area 25 Building 3124 Leachfield Characterization

(Page 1 of 3 )

\begin{tabular}{|c|c|c|c|c|c|}
\hline $\begin{array}{c}\text { Sample } \\
\text { Location }\end{array}$ & $\begin{array}{l}\text { Sample } \\
\text { Number }\end{array}$ & $\begin{array}{l}\text { Depth Below } \\
\text { Leachrock (ft) }\end{array}$ & Matrix & Sample Type & Analyses \\
\hline NA & TCA00001 & NA & Water & Trip Blank & Total VOCs \\
\hline NA & TCA00002 & NA & Water & Trip Blank & Total VOCs \\
\hline NA & TCA00004 & NA & Water & Trip Blank & Total VOCs \\
\hline NA & TCA00006 & NA & Water & Trip Blank & Total VOCs \\
\hline Septic Tank & TCA60002 & NA & Sludge & $\begin{array}{c}\text { MS/MSD } \\
\text { Environmental }\end{array}$ & $\begin{array}{c}\text { Set 1, Rad 1, } \\
\text { TCLP }\end{array}$ \\
\hline Distribution Box & TCA60003 & NA & Sludge & Environmental & $\begin{array}{c}\text { Set 1, Rad 1, } \\
\text { TCLP }\end{array}$ \\
\hline Septic Tank & TCA60004 & NA & Liquid & $\begin{array}{c}\text { MS/MSD } \\
\text { Environmental }\end{array}$ & Set 1, Rad 1 \\
\hline NA & TCA60006 & NA & Water & $\begin{array}{c}\text { Equipment } \\
\text { Rinsate Blank - } \\
\text { Beakers and } \\
\text { Scoops }\end{array}$ & Set 1, Rad 1 \\
\hline NA & TCA60007 & NA & Water & Field Blank & Set $1, \operatorname{Rad} 1$ \\
\hline Septic Tank & TCA60008 & NA & Liquid & $\begin{array}{l}\text { Field Duplicate of } \\
\text { TCA60004 }\end{array}$ & Set 1 , Rad 1 \\
\hline \multirow{9}{*}{ NA } & TCA60010 & NA & Water & Trip Blank & Total VOCs \\
\hline & TCA60011 & NA & Water & $\begin{array}{c}\text { Equipment } \\
\text { Rinsate -Bowl }\end{array}$ & Set 1, Rad 1 \\
\hline & TCA60012 & NA & Water & Trip Blank & Total VOCs \\
\hline & TCA60013 & NA & Water & $\begin{array}{c}\text { Source } \\
\text { Blank-Poly- } \\
\text { urethane Bag }\end{array}$ & Set $1, \operatorname{Rad} 1$ \\
\hline & TCA60014 & NA & Water & Trip Blank & Total VOCs \\
\hline & TCA60015 & NA & Water & Field Blank & Set $1, \operatorname{Rad} 1$ \\
\hline & TCA60016 & NA & Water & Trip Blank & Total VOCs \\
\hline & TCA60017 & NA & Water & $\begin{array}{c}\text { Equipment } \\
\text { Rinsate - Core } \\
\text { Barrels }\end{array}$ & Set $1, \operatorname{Rad} 1$ \\
\hline & TCA60018 & NA & Water & Trip Blank & Total VOCs \\
\hline \multirow[t]{2}{*}{ L-1.3 } & TCA60019 & $0-1$ & Soil & Environmental & Set $1, \operatorname{Rad} 1$ \\
\hline & TCA60020 & $2.5-3.5$ & Soil & Environmental & Set $1, \operatorname{Rad} 1$ \\
\hline
\end{tabular}


Table A.3-1

Samples Collected During the CAU 266 Area 25 Building 3124 Leachfield Characterization

(Page 2 of 3 )

\begin{tabular}{|c|c|c|c|c|c|}
\hline $\begin{array}{l}\text { Sample } \\
\text { Location }\end{array}$ & $\begin{array}{l}\text { Sample } \\
\text { Number }\end{array}$ & $\begin{array}{l}\text { Depth Below } \\
\text { Leachrock (ft) }\end{array}$ & Matrix & Sample Type & Analyses \\
\hline $\mathrm{L}-1.2$ & TCA60021 & $0-1$ & Soil & Environmental & Set $1, \operatorname{Rad} 1$ \\
\hline $\mathrm{L}-1.2$ & TCA60022 & $2.5-3.5$ & Soil & Environmental & Set 1 \\
\hline \multirow{2}{*}{$\mathrm{L}-1.1$} & TCA60023 & $0-1$ & Soil & Environmental & Set 1 \\
\hline & TCA60024 & $2.5-3.5$ & Soil & Environmental & Set 1 \\
\hline \multirow{4}{*}{ L-2.3 } & TCA60025 & $0-1$ & Soil & Environmental & Set $1, \operatorname{Rad} 1$ \\
\hline & TCA60026 & $2.5-3.5$ & Soil & Environmental & Set $1, \operatorname{Rad} 1$ \\
\hline & TCA60027 & $2.5-3.5$ & Soil & Field Duplicate & Set $1, \operatorname{Rad} 1$ \\
\hline & TCA60030 & $7.5-8.5$ & Soil & Environmental & Not Used \\
\hline NA & TCA60028 & NA & Water & Trip Blank & Total VOCs \\
\hline \multirow{2}{*}{$\mathrm{L}-2.2$} & TCA60031 & $0-1$ & Soil & Environmental & Set 1 \\
\hline & TCA60032 & $2.5-3.5$ & Soil & Environmental & Set 1 \\
\hline \multirow{2}{*}{$\mathrm{L}-2.1$} & TCA60033 & $0-1$ & Soil & Environmental & Set 1 \\
\hline & TCA60034 & $2.5-3.5$ & Soil & $\begin{array}{c}\text { MS/MSD } \\
\text { Environmental }\end{array}$ & Set 1, Rad 1 \\
\hline NA & TCA60035 & NA & Water & Trip Blank & Total VOCs \\
\hline \multirow{2}{*}{ L-3.3 } & TCA60036 & $0-1$ & Soil & Environmental & Set 1 \\
\hline & TCA60037 & $2.5-3.5$ & Soil & Environmental & Set 1 \\
\hline \multirow{2}{*}{ L-3.2 } & TCA60038 & $0-1$ & Soil & Environmental & Set 1 \\
\hline & TCA60039 & $2.5-3.5$ & Soil & Environmental & Set 1 \\
\hline \multirow{2}{*}{ L-3.1 } & TCA60040 & $0-1$ & Soil & Environmental & Set 1, Rad 1 \\
\hline & TCA60041 & $2.5-3.5$ & Soil & Environmental & Set 1, Rad 1 \\
\hline DC-1 & TCA60042 & $5-6$ bgs & Soil & Environmental & Set 1 \\
\hline \multirow{2}{*}{ NA } & TCA60043 & NA & Water & Trip Blank & Total VOCs \\
\hline & TCA60044 & NA & Water & Field Blank & Set $1, \operatorname{Rad} 1$ \\
\hline ST-3 & TCA60045 & $5-6$ bgs & Soil & Environmental & Set 1 \\
\hline \multirow{2}{*}{ NA } & TCA60046 & NA & Water & Trip Blank & Total VOCs \\
\hline & TCA60047 & NA & Water & Trip Blank & Total VOCs \\
\hline
\end{tabular}


Table A.3-1

\section{Samples Collected During the CAU 266 Area 25 Building 3124 Leachfield Characterization}

(Page 3 of 3)

\begin{tabular}{|c|c|c|c|c|c|}
\hline $\begin{array}{l}\text { Sample } \\
\text { Location }\end{array}$ & $\begin{array}{l}\text { Sample } \\
\text { Number }\end{array}$ & $\begin{array}{l}\text { Depth Below } \\
\text { Leachrock (ft) }\end{array}$ & Matrix & Sample Type & Analyses \\
\hline \multirow{3}{*}{ L-4.3 } & TCA60048 & $0-1$ & Soil & Environmental & Set $1, \operatorname{Rad} 1$ \\
\hline & TCA60049 & $0-1$ & Soil & Field Duplicate & Set 1, Rad 1 \\
\hline & TCA60050 & $2.5-3.5$ & Soil & Environmental & Set 1 \\
\hline \multirow{2}{*}{ L-4.2 } & TCA60051 & $0-1$ & Soil & Environmental & Set 1 \\
\hline & TCA60052 & $2.5-3.5$ & Soil & Environmental & Set 1 \\
\hline \multirow{2}{*}{$\mathrm{L}-4.1$} & TCA60053 & $0-1$ & Soil & $\begin{array}{c}\text { MS/MSD } \\
\text { Environmental }\end{array}$ & Set $1, \operatorname{Rad} 1$ \\
\hline & TCA60054 & $2.5-3.5$ & Soil & Environmental & Set 1 \\
\hline ST-1 & TCA60055 & $5-6$ bgs & Soil & Environmental & Set 1 \\
\hline NA & TCA60056 & NA & Water & Trip Blank & Total VOCs \\
\hline $\begin{array}{c}\text { Drum\# } \\
\text { CAU266-99-002 }\end{array}$ & TCA60057 & NA & Liquid & $\begin{array}{c}\text { Waste } \\
\text { Characterization }\end{array}$ & Set 1, Rad 1 \\
\hline $\begin{array}{l}\text { Between L-3.1 } \\
\text { and L-4.1 }\end{array}$ & TCA60058 & $5-7$ bgs & Soil & $\begin{array}{l}\text { Geotechnical \& } \\
\text { Bioassessment }\end{array}$ & None Performed \\
\hline BCK5 & TCA10110 & $0-0.5 \mathrm{bgs}$ & Soil & Background & Set 2 \\
\hline BCK6 & TCA10111 & $0-0.5 \mathrm{bgs}$ & Soil & Background & Set 2 \\
\hline BCK17 & TCA10112 & $0-0.5 \mathrm{bgs}$ & Soil & Background & Set 2 \\
\hline BCK3 & TCA10113 & $0-0.5$ bgs & Soil & Background & Set 2 \\
\hline BCK1 & TCA10114 & $0-0.5 \mathrm{bgs}$ & Soil & Background & Set 2 \\
\hline $\mathrm{BCK} 2$ & TCA10115 & $0-0.5 \mathrm{bgs}$ & Soil & Background & Set 2 \\
\hline
\end{tabular}

Set 1: Analytical parameters are total VOC, total SVOC, TPH-Diesel/Oil, total RCRA metals, PCBs

Set 2: Total RCRA metals, gamma spectrometry, strontium-90, and isotopic plutonium, uranium, and americium

Rad 1: Gamma spectrometry; strontium-90; and isotopic uranium, plutonium, americium

Rad 2: Gamma spectrometry, strontium-90, isotopic uranium

TCLP $=$ Toxicity Characteristic Leaching Procedure (VOCs, SVOCs, RCRA metals)

MS/MSD = Matrix spike/matrix spike duplicate

$\mathrm{NA}=$ Not Applicable

$\mathrm{VOC}=$ Volatile organic compounds

$\mathrm{TPH}=$ Total petroleum hydrocarbons

SVOC $=$ Semivolatile organic compounds

RCRA $=$ Resource Conservation and Recovery Act

bgs $=$ Below ground surface (in feet)

$\mathrm{ft}=$ Feet 
Table A.3-2

\section{Laboratory Analytical Methods Used for Samples Collected at the CAU 266 Area 25 Building 3124 Leachfield Characterization}

\begin{tabular}{|c|c|}
\hline Analytical Parameter & Analytical Method \\
\hline Total volatile organic compounds & EPA $8260 \mathrm{~B}^{\mathrm{a}}$ \\
\hline TCLP volatile organic compounds & EPA $1311 / 8260 B^{a}$ \\
\hline Total semivolatile organic compounds & EPA $8270 C^{a}$ \\
\hline TCLP semivolatile organic compounds & EPA $1311 / 8270 C^{a}$ \\
\hline Total petroleum hydrocarbons - diesel range & EPA 8015B (modified) $)^{\mathrm{a}}$ \\
\hline $\begin{array}{l}\text { Total RCRA metals (arsenic, barium, cadmium, chromium, lead, selenium, silver, } \\
\text { and mercury) }\end{array}$ & $\begin{array}{l}\text { EPA } 6010 B / 7470 A^{a} \\
\text { EPA } 6010 B / 7471 A^{a}\end{array}$ \\
\hline $\begin{array}{l}\text { TCLP RCRA metals (arsenic, barium, cadmium, chromium, lead, selenium, silver, } \\
\text { and mercury) }\end{array}$ & EPA $1311 / 6010 B / 7470 A^{a}$ \\
\hline Polychlorinated biphenyls & EPA $8082^{a}$ \\
\hline Gamma-emitting radionuclides & $\begin{array}{l}\text { L-E10.602.PC } \\
\text { SOP } 739 / 713^{b, f},\end{array}$ \\
\hline Isotopic uranium & $\begin{array}{l}\text { L-E10.605.PL }{ }^{\mathrm{b}, \mathrm{d}, \mathrm{e}}(\text { (soil) } \\
\text { PAl } 777 / 778 / 714^{\mathrm{b}, \mathrm{f}} \text { (water) }\end{array}$ \\
\hline Isotopic plutonium & $\begin{array}{l}\text { L-E10.601.PL,c,e } \\
\text { PAI } 777 / 782 / 714^{\mathrm{b}, \mathrm{c}, \mathrm{f}}\end{array}$ \\
\hline Strontium-90 & $\begin{array}{l}\text { L-E10.610.PL b,e } \\
\text { PAI } 717 / 724^{b, f}\end{array}$ \\
\hline Isotopic americium & PAI $780 / 714^{\mathrm{b}, \mathrm{f}}$ \\
\hline
\end{tabular}

${ }^{a}$ EPA Test Methods for Evaluating Solid Waste, 3rd Edition, Parts 1-4, SW-846 (EPA, 1996)

${ }^{\mathrm{b}}$ Or equivalent method

'Separation and Preconcentration of Actinides from Acidic Media by Extraction Chromatography (Horwitz, et al., 1993)

${ }^{d}$ Separation and Preconcentration of Uranium from Acidic Media by Extraction Chromatography (Horwitz, et al., 1992)

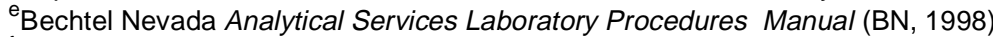

${ }^{f}$ Paragon Analytics, Inc. Standard Operating Procedures Manual (PAI, 1998)

\section{A.3.1 Total Volatile Organic Compound Analytical Results}

The total VOC analytical results for soil samples detected above minimum reporting limits established in the Work Plan for Leachfield CAUs (DOE/NV, 1998), along with the associated PALs, are presented in Table A.3-3. None of these results exceed the PALs (DOE/NV, 1998).

A portion of the results for acrolein and 2-chloroethyl-vinyl ether were rejected for samples collected. Acrolein is most commonly used as an herbicide in irrigation canals (EPA, 1989). It is also used as a 
Table A.3-3

Soil Sample Results for Total Volatile Organic Compounds Detected Above Minimum Reporting Limits ${ }^{b}$, CAU 266 Area 25 Building 3124 Leachfield

\begin{tabular}{|c|c|c|c|c|c|}
\hline \multirow{3}{*}{$\begin{array}{l}\text { Sampling } \\
\text { Location }\end{array}$} & \multirow{2}{*}{$\begin{array}{l}\text { Sample } \\
\text { Number }\end{array}$} & \multirow{2}{*}{$\begin{array}{l}\text { Start Depth } \\
\text { Below } \\
\text { Leachrock (ft) }\end{array}$} & \multirow{2}{*}{$\begin{array}{l}\text { End Depth } \\
\text { Below } \\
\text { Leachrock (ft) }\end{array}$} & \multicolumn{2}{|c|}{$\begin{array}{l}\text { Contaminants of Potential Concern } \\
\qquad(\mu \mathrm{g} / \mathrm{kg})\end{array}$} \\
\hline & & & & 2-Butanone ${ }^{b}$ & Acetone $^{b}$ \\
\hline & \multicolumn{3}{|c|}{ Preliminary Action Levels ${ }^{\mathrm{a}}(\mu \mathrm{g} / \mathrm{kg})$} & $27,000,000$ & $6,100,000$ \\
\hline L-1.3 & TCA60019 & 0 & 1 & -- & $7.8(\mathrm{~J})$ \\
\hline L-2.3 & TCA60025 & 0 & 1 & -- & $9.2(\mathrm{~J})$ \\
\hline L-3-3 & TCA60036 & 0 & 1 & $5(\mathrm{~J})$ & -- \\
\hline$L-3.2$ & TCA60038 & 0 & 1 & -- & $9(J)$ \\
\hline \multirow{3}{*}{ L-4.3 } & TCA60048 & 0 & 1 & -- & $8.1(\mathrm{~J})$ \\
\hline & TCA60049 & 0 & 1 & -- & $10(\mathrm{~J})$ \\
\hline & TCA60050 & 2.5 & 3.5 & -- & $9.8(\mathrm{~J})$ \\
\hline L-4.1 & TCA60053 & 0 & 1 & -- & $8.7(\mathrm{~J})$ \\
\hline
\end{tabular}

${ }^{a}$ Environmental Protection Agency Region 9, Industrial Preliminary Remediation Goals (PRGs) (EPA, 1998)

${ }^{\mathrm{b}}$ Values below the analyte specific minimum reporting limit of $20 \mu \mathrm{g} / \mathrm{kg}$ are reported here for Acetone and 2-Butanone because

they were reported in the draft

$--=$ Not detected above minimum reporting limit

$\mathrm{J}=$ Estimated value

$\mu \mathrm{g} / \mathrm{km}=$ Micrograms per kilogram

$\mathrm{ft}=$ Feet

pesticide. 2-chloroethyl-vinyl ether is used in the manufacture of anesthetics, sedatives, and cellulose ethers (Spectrum Laboratories, 1999). Acrolein and 2-chloroethyl-vinyl ether are highly volatile and are not persistent in the environment and do not concentrate in sediments (EC, 1999; Spectrum Laboratories, 1999). This is an acceptable data gap because acrolein and 2-chloroethyl-vinyl ether is not expected at this CAU and it was not detected in other usable results.

\section{A.3.2 Total Semivolatile Organic Compound Analytical Results}

The laboratory results for SVOCs for soil samples indicate that contaminants were not present above the minimum reporting limits as established in the Work Plan for Leachfield CAUs (DOE/NV, 1998). 


\section{A.3.3 Total Petroleum Hydrocarbon Results}

Total petroleum hydrocarbons were not detected in soil in the diesel range above the minimum reporting limits as established in the CAIP (DOE/NV, 1999).

\section{A.3.4 Total RCRA Metals Results}

The total RCRA metals detected in soil above the minimum reporting limits (DOE/NV, 1999) are presented in Table A.3-4. The total RCRA metals detected at the site were arsenic, barium, chromium, lead, mercury, and selenium. The total RCRA metals results were all below the PALs (DOE/NV, 1999; EPA, 1998) except for arsenic. Arsenic was detected at several sampling locations above the Industrial Soil Preliminary Remediation Goal (PRG) of $3.0 \mathrm{mg} / \mathrm{kg}$. These results are lower than the 7 to $8 \mathrm{ppm}(\mathrm{mg} / \mathrm{kg})$ mean concentration of arsenic in silt from the Nellis Air Force Range (NBMG, 1998; Moore, 1999). Arsenic was detected in the six background soil samples collected from the surface area near Test Cell A (see Figure 1 in Appendix B). These soil samples were found to have slightly higher arsenic concentrations than the planned soil sampling locations chosen for the investigation. The concentrations at CAU 266 are not unusual and are considered representative of ambient conditions.

\section{A.3.5 PCB Analytical Results}

The laboratory PCB results for soil samples detected above the minimum reporting limits (DOE/NV, 1999), along with the associated PALs are presented in Table A.3-5. None of these results exceed the PALs (DOE/NV, 1999).

\section{A.3.6 Gamma Spectrometry Results}

Gamma spectrometry results for soil samples detected above the minimum reporting limits as specified in the CAIP (DOE/NV, 1999) are presented in Table A.3-6. The radiological results were not distinguishable from background concentrations listed in the Off-Site Radiation Exposure Review Project (McArthur and Miller, 1989) or the Environmental Monitoring Report for the Proposed Ward Valley California Low Level Radioactive Waste (LLRW) Facility (Atlan-Tech, 1992). 
Table A.3-4

Soil Samples for Total RCRA Metals Detected Above Minimum Reporting Limits Corrective Action Unit 266 Area 25 Building 3124 Leachfield, Nevada Test Site (Page 1 of 2 )

\begin{tabular}{|c|c|c|c|c|c|c|c|c|c|}
\hline \multirow{2}{*}{$\begin{array}{l}\text { Sample } \\
\text { Location }\end{array}$} & \multirow{2}{*}{$\begin{array}{l}\text { Sample } \\
\text { Number }\end{array}$} & \multirow{2}{*}{$\begin{array}{c}\text { Start } \\
\text { Depth } \\
\text { Below } \\
\text { Leachrock } \\
\text { (ft) }\end{array}$} & \multirow{2}{*}{$\begin{array}{l}\text { End Depth } \\
\text { Below } \\
\text { Leachrock } \\
\text { (ft) }\end{array}$} & \multicolumn{6}{|c|}{ Contaminants of Potential Concern (mg/kg) } \\
\hline & & & & Arsenic & Barium & Chromium & Lead & Mercury & Selenium \\
\hline \multicolumn{4}{|c|}{ Preliminary Action Levels ${ }^{a}$ (mg/kg) } & 3.0 & 100,000 & 64 & 1,000 & 560 & 9,400 \\
\hline \multirow{2}{*}{ L-1.3 } & TCA60019 & 0 & 1 & $3.4(J)^{b}$ & $67.3(\mathrm{~J})^{\mathrm{d}}$ & $3.8(J)^{b, d}$ & 6 & -- & -- \\
\hline & TCA60020 & 2.5 & 3.5 & $3.2(\mathrm{~J})^{\mathrm{b}}$ & $108(J)^{d}$ & $3.4(J)^{b, d}$ & 5.6 & -- & -- \\
\hline \multirow{2}{*}{$\mathrm{L}-1.2$} & TCA60021 & 0 & 1 & $3.6(J)^{b}$ & $111(J)^{d}$ & $4.2(J)^{b, d}$ & 5.9 & -- & -- \\
\hline & TCA60022 & 2.5 & 3.5 & $3.4(\mathrm{~J})^{\mathrm{b}}$ & $105(\mathrm{~J})^{\mathrm{d}}$ & $3.8(J)^{b, d}$ & 5.8 & -- & -- \\
\hline \multirow{2}{*}{$\mathrm{L}-1.1$} & TCA60023 & 0 & 1 & $2.5(J)^{b}$ & $74.2(\mathrm{~J})^{d}$ & $3.5(J)^{b, d}$ & 6.3 & -- & -- \\
\hline & TCA60024 & 2.5 & 3.5 & $2.8(\mathrm{~J})^{\mathrm{b}}$ & $118(J)^{d}$ & $3.8(J)^{b, d}$ & 5.7 & -- & -- \\
\hline \multirow{3}{*}{ L-2.3 } & TCA60025 & 0 & 1 & $2.5(\mathrm{~J})^{\mathrm{b}}$ & $70.8(\mathrm{~J})^{d}$ & $4(J)^{b, d}$ & 5.9 & -- & -- \\
\hline & TCA60026 & 2.5 & 3.5 & $3.1(\mathrm{~J})^{\mathrm{b}}$ & $104(\mathrm{~J})^{\mathrm{d}}$ & $3.5(J)^{b, d}$ & 5.4 & 0.11 & -- \\
\hline & TCA60027 & 2.5 & 3.5 & $3.1(\mathrm{~J})^{\mathrm{b}}$ & $93.4(J)^{d}$ & $3.2(J)^{b, d}$ & 7 & -- & -- \\
\hline \multirow{2}{*}{ L-2.2 } & TCA60031 & 0 & 1 & $2.7(J)^{b}$ & 84 & $3.8(J)^{b}$ & $7(\mathrm{~J})^{\mathrm{c}, \mathrm{e}}$ & -- & -- \\
\hline & TCA60032 & 2.5 & 3.5 & $3.1(\mathrm{~J})^{\mathrm{b}}$ & 93.2 & $2.7(J)^{b}$ & $6(J)^{c, e}$ & -- & -- \\
\hline \multirow{2}{*}{ L-2.1 } & TCA60033 & 0 & 1 & $2.8(J)^{b}$ & 108 & $14(J)^{b}$ & $8.6(J)^{\mathrm{c}, \mathrm{e}}$ & -- & -- \\
\hline & TCA60034 & 0 & 1 & $3(J)^{b}$ & 85.6 & $4.7(J)^{b}$ & $6.5(J)^{\mathrm{c}, \mathrm{e}}$ & -- & -- \\
\hline \multirow{2}{*}{ L-3.3 } & TCA60036 & 0 & 1 & $2.7(\mathrm{~J})^{\mathrm{b}}$ & 161 & $6.3(\mathrm{~J})^{\mathrm{b}}$ & $9.3(\mathrm{~J})^{\mathrm{c}, \mathrm{e}}$ & -- & 0.53 \\
\hline & TCA60037 & 2.5 & 3.5 & $2.7(J)^{b}$ & 94.3 & $3.5(J)^{b}$ & $9.3(\mathrm{~J})^{\mathrm{c}, \mathrm{e}}$ & -- & -- \\
\hline \multirow{2}{*}{ L-3.2 } & TCA60038 & 0 & 1 & $3.2(\mathrm{~J})^{\mathrm{b}}$ & 142 & $9.2(\mathrm{~J})^{\mathrm{b}}$ & $9.6(J)^{c, e}$ & -- & -- \\
\hline & TCA60039 & 2.5 & 3.5 & $3.3(\mathrm{~J})^{\mathrm{b}}$ & 105 & $4(J)^{b}$ & $6.4(J)^{c, e}$ & -- & -- \\
\hline \multirow{2}{*}{ L-3.1 } & TCA60040 & 0 & 1 & $2.8(\mathrm{~J})^{\mathrm{b}}$ & 66 & $28.8(J)^{b}$ & $13.4(J)^{c, e}$ & 0.1 & -- \\
\hline & TCA60041 & 2.5 & 3.5 & $2.7(J)^{b}$ & 95 & $3.6(J)^{b}$ & $5.9(\mathrm{~J})^{\mathrm{c}, \mathrm{e}}$ & -- & -- \\
\hline DC-1 & TCA60042 & 5 bgs & 6 bgs & $3.7(\mathrm{~J})^{\mathrm{b}}$ & 120 & $3.5(\mathrm{~J})^{\mathrm{b}}$ & $6.2(J)^{\mathrm{c}, \mathrm{e}}$ & -- & -- \\
\hline ST-3 & TCA60045 & 5 bgs & 6 bgs & $2.6(J)^{b}$ & 134 & $3(J)^{b}$ & $5.2(J)^{c, e}$ & -- & -- \\
\hline \multirow{3}{*}{ L-4.3 } & TCA60048 & 0 & 1 & $3.5(J)^{b}$ & 124 & $5.1(\mathrm{~J})^{\mathrm{b}}$ & $8.4(J)^{\mathrm{c}, \mathrm{e}}$ & -- & -- \\
\hline & TCA60049 & 0 & 1 & $3.6(J)^{b}$ & 93.6 & $4.1(\mathrm{~J})^{\mathrm{b}}$ & $5.8(J)^{c, e}$ & -- & -- \\
\hline & TCA60050 & 2.5 & 3.5 & $2.8(\mathrm{~J})^{\mathrm{b}}$ & 85.4 & $2.6(J)^{b}$ & $5.2(J)^{c, e}$ & -- & -- \\
\hline
\end{tabular}


Table A.3-4

\section{Soil Samples for Total RCRA Metals Detected Above Minimum Reporting Limits Corrective Action Unit 266 Area 25 Building 3124 Leachfield, Nevada Test Site} (Page 2 of 2 )

\begin{tabular}{|c|c|c|c|c|c|c|c|c|c|}
\hline \multirow{2}{*}{$\begin{array}{l}\text { Sample } \\
\text { Location }\end{array}$} & \multirow{2}{*}{$\begin{array}{l}\text { Sample } \\
\text { Number }\end{array}$} & \multirow{2}{*}{$\begin{array}{c}\text { Start } \\
\text { Depth } \\
\text { Below } \\
\text { Leachrock } \\
\text { (ft) }\end{array}$} & \multirow{2}{*}{$\begin{array}{l}\text { End Depth } \\
\text { Below } \\
\text { Leachrock } \\
\text { (ft) }\end{array}$} & \multicolumn{6}{|c|}{ Contaminants of Potential Concern $(\mathrm{mg} / \mathrm{kg})$} \\
\hline & & & & Arsenic & Barium & Chromium & Lead & Mercury & Selenium \\
\hline \multicolumn{4}{|c|}{ Preliminary Action Levels ${ }^{\mathrm{a}}(\mathrm{mg} / \mathrm{kg})$} & 3.0 & 100,000 & 64 & 1,000 & 560 & 9,400 \\
\hline \multirow{2}{*}{$\mathrm{L}-4.2$} & TCA60051 & 0 & 1 & $3.4(\mathrm{~J})^{\mathrm{b}}$ & 114 & $4.1(\mathrm{~J})^{\mathrm{b}}$ & 6.8 & -- & -- \\
\hline & TCA60052 & 2.5 & 3.5 & $3.3(J)^{b}$ & 140 & $3.4(J)^{b}$ & 5.4 & -- & -- \\
\hline \multirow{2}{*}{ L-4.1 } & TCA60053 & 0 & 1 & $3.3(J)^{b}$ & 109 & $4.9(J)^{b}$ & 13.3 & -- & -- \\
\hline & TCA60054 & 2.5 & 3.5 & $2.9(\mathrm{~J})^{\mathrm{b}}$ & 116 & $2.8(J)^{b}$ & 6.5 & -- & -- \\
\hline ST-1 & TCA60055 & 5 bgs & 6 bgs & $3(J)^{b}$ & 116 & $3.1(\mathrm{~J})^{\mathrm{b}}$ & 5.7 & -- & -- \\
\hline
\end{tabular}

${ }^{\text {a} E n v i r o n m e n t a l ~ P r o t e c t i o n ~ A g e n c y ~ R e g i o n ~ 9, ~ I n d u s t r i a l ~ P r e l i m i n a r y ~ R e m e d i a t i o n ~ G o a l s ~(P R G s) ~(E P A, ~ 1998) ~}$

${ }^{b}$ Duplicate precision analyses were outside control limits

${ }^{c}$ CRI/CRA \% recovery criteria not met

${ }^{d}$ Serial dilution $\% D$ was outside control limits

${ }^{\mathrm{e}}$ Low CRI standard recovery

$--=$ Not detected above minimum reporting limit

$\mathrm{J}=$ Estimated value

bgs $=$ Below ground surface (in feet)

$\mathrm{mg} / \mathrm{kg}=$ Milligrams per kilogram

$\mathrm{ft}=$ Feet

Table A.3-5

Soil Sample Results for PCBs Detected Above Minimum Reporting Limits, CAU 266 Area 25 Building 3124 Leachfield

\begin{tabular}{|c|c|c|c|c|c|}
\hline \multirow{3}{*}{$\begin{array}{l}\text { Sample } \\
\text { Location }\end{array}$} & \multirow{2}{*}{$\begin{array}{l}\text { Sample } \\
\text { Number }\end{array}$} & \multirow{2}{*}{$\begin{array}{l}\text { Start Depth } \\
\text { Below } \\
\text { Leachrock (ft) }\end{array}$} & \multirow{2}{*}{$\begin{array}{l}\text { End Depth } \\
\text { Below } \\
\text { Leachrock (ft) }\end{array}$} & \multicolumn{2}{|c|}{$\begin{array}{l}\text { Contaminants of Potential Concern } \\
\qquad(\mu \mathrm{g} / \mathrm{kg})\end{array}$} \\
\hline & & & & Aroclor-1254 & Aroclor-1260 \\
\hline & \multicolumn{3}{|c|}{ Preliminary Action Levels ${ }^{a}(\mu \mathrm{g} / \mathrm{kg})$} & 1,300 & 1,300 \\
\hline L-2.1 & TCA60033 & 0 & 1 & 42 & -- \\
\hline L-3.2 & TCA60038 & 0 & 1 & 46 & 41 \\
\hline L-3.1 & TCA60040 & 0 & 1 & 69 & 67 \\
\hline
\end{tabular}

${ }^{\text {a} E n v i r o n m e n t a l ~ P r o t e c t i o n ~ A g e n c y ~ R e g i o n ~ 9, ~ I n d u s t r i a l ~ P r e l i m i n a r y ~ R e m e d i a t i o n ~ G o a l s ~(P R G s) ~(E P A, ~ 1998) ~}$

$\mathrm{ft}=$ Feet

$--=$ Not detected above minimum reporting limit

$\mathrm{J}=$ Estimated value

bgs = Below Ground Surface (in feet)

$\mu \mathrm{g} / \mathrm{km}=$ Micrograms per kilogram 
Table A.3-6

Soil Sample Results for Gamma-Emitting Radionuclides Above MRLs, CAU 266 Area 25 Building 3124 Leachfield

\begin{tabular}{|c|c|c|c|c|c|c|c|c|c|c|c|}
\hline \multirow{3}{*}{$\begin{array}{l}\text { Sample } \\
\text { Location }\end{array}$} & \multirow{2}{*}{$\begin{array}{l}\text { Sample } \\
\text { Number }\end{array}$} & \multirow{2}{*}{$\begin{array}{c}\text { Start } \\
\text { Depth } \\
\text { Below } \\
\text { Leachrock } \\
\text { (ft) }\end{array}$} & \multirow{2}{*}{$\begin{array}{l}\text { End Depth } \\
\text { Below } \\
\text { Leachrock } \\
\text { (ft) }\end{array}$} & \multicolumn{8}{|c|}{ Contaminants of Potential Concern $(\mathrm{pCi} / \mathrm{g})$} \\
\hline & & & & Actinium-228 & Bismuth-212 & Bismuth-214 & Cesium-137 & Potassium-40 & Lead-212 & Lead-214 & Thalium-208 \\
\hline & \multicolumn{3}{|c|}{ Background Concentrations $\mathrm{s}^{\mathrm{a}, \mathrm{b}}$} & $0.4-3.64^{\mathrm{a}}$ & $0.49-2.4^{b}$ & $0.1-3.47^{b}$ & $0.4-7.0^{b}$ & $11-96^{a}$ & $0.86-2.9^{a}$ & $0.5-2.9^{b}$ & $0.5-3.4^{\mathrm{a}}$ \\
\hline \multirow{2}{*}{$\mathrm{L}-1.3$} & TCA60019 & 0 & 1 & $1.01 \pm 0.30(\mathrm{~J})$ & -- & $0.63 \pm 0.23(\mathrm{~J})$ & -- & $17.60 \pm 3.7(\mathrm{~J})$ & $1 \pm 0.24(\mathrm{~J})$ & $0.67 \pm 0.19(\mathrm{~J})$ & -- \\
\hline & TCA60020 & 2.5 & 3.5 & $2.02 \pm 0.47(\mathrm{~J})$ & -- & $0.9 \pm 0.27(\mathrm{~J})$ & -- & $31.4 \pm 6(\mathrm{~J})$ & $2.13 \pm 0.41(\mathrm{~J})$ & $1.13 \pm 0.26(\mathrm{~J})$ & $0.56 \pm 0.16(\mathrm{~J})$ \\
\hline $\mathrm{L}-1.2$ & TCA60021 & 0 & 1 & $1.83 \pm 0.43(\mathrm{~J})$ & $2.40 \pm 1.20(\mathrm{~J})$ & $0.97 \pm 0.26(\mathrm{~J})$ & -- & $28.6 \pm 5.5(\mathrm{~J})$ & $1.74 \pm 0.34(\mathrm{~J})$ & $0.97 \pm 0.24(\mathrm{~J})$ & $0.69 \pm 0.17(\mathrm{~J})$ \\
\hline \multirow{3}{*}{ L-2.3 } & TCA60025 & 0 & 1 & $1.77 \pm 0.44(\mathrm{~J})$ & $2.70 \pm 1.40(\mathrm{~J})$ & $0.82 \pm 0.29(\mathrm{~J})$ & -- & $28.7 \pm 5.6(\mathrm{~J})$ & $1.7 \pm 0.35(\mathrm{~J})$ & $0.8 \pm 0.22(\mathrm{~J})$ & $0.56 \pm 0.17(\mathrm{~J})$ \\
\hline & TCA60026 & 2.5 & 3.5 & $1.73 \pm 0.46(\mathrm{~J})$ & $2.90 \pm 1.40(\mathrm{~J})$ & $1.07 \pm 0.32(\mathrm{~J})$ & -- & $32.3 \pm 6.2(\mathrm{~J})$ & $2 \pm 0.39(\mathrm{~J})$ & $1.02 \pm 0.26(\mathrm{~J})$ & $0.58 \pm 0.16(\mathrm{~J})$ \\
\hline & TCA60027 & 2.5 & 3.5 & $2.07 \pm 0.52(\mathrm{~J})$ & -- & $0.99 \pm 0.31(\mathrm{~J})$ & -- & $33 \pm 6.4(\mathrm{~J})$ & $2.02 \pm 0.41(\mathrm{~J})$ & $1.06 \pm 0.27(\mathrm{~J})$ & $0.68 \pm 0.19(\mathrm{~J})$ \\
\hline $\mathrm{L}-2.1$ & TCA60034 & 0 & 1 & $1.82 \pm 0.44(\mathrm{~J})$ & -- & $0.91 \pm 0.27(\mathrm{~J})$ & -- & $27.2 \pm 5.3(\mathrm{~J})$ & $1.74 \pm 0.34(\mathrm{~J})$ & $0.75 \pm 0.21(\mathrm{~J})$ & $0.57 \pm 0.16(\mathrm{~J})$ \\
\hline \multirow{2}{*}{ L-3.1 } & TCA60040 & 0 & 1 & $1.51 \pm 0.46(\mathrm{~J})$ & -- & $0.86 \pm 0.30(\mathrm{~J})$ & $0.33 \pm 0.14(\mathrm{~J})$ & $32.7 \pm 6.4(\mathrm{~J})$ & $1.81 \pm 0.37(\mathrm{~J})$ & $1.18 \pm 0.28(\mathrm{~J})$ & $0.69 \pm 0.19(\mathrm{~J})$ \\
\hline & TCA60041 & 2.5 & 3.5 & $1.71 \pm 0.46(\mathrm{~J})$ & -- & $0.87 \pm 0.31(\mathrm{~J})$ & -- & $32.3 \pm 6.2(\mathrm{~J})$ & $1.84 \pm 0.37(\mathrm{~J})$ & $0.78 \pm 0.24(\mathrm{~J})$ & $0.61 \pm 0.18(\mathrm{~J})$ \\
\hline \multirow{2}{*}{ L-4.3 } & TCA60048 & 0 & 1 & $1.77 \pm 0.46(\mathrm{~J})$ & $2.90 \pm 1.50(\mathrm{~J})$ & $0.97 \pm 0.30(\mathrm{~J})$ & -- & $28.3 \pm 5.6(\mathrm{~J})$ & $1.62 \pm 0.34(\mathrm{~J})$ & $1.14 \pm 0.27(\mathrm{~J})$ & $0.55 \pm 0.17(\mathrm{~J})$ \\
\hline & TCA60049 & 0 & 1 & $1.71 \pm 0.47(\mathrm{~J})$ & -- & $1.15 \pm 0.35(\mathrm{~J})$ & -- & $31.8 \pm 6.2(\mathrm{~J})$ & $1.98 \pm 0.4(\mathrm{~J})$ & $1.2 \pm 0.3(\mathrm{~J})$ & -- \\
\hline L-4.1 & TCA60053 & 0 & 1 & $1.99 \pm 0.50(\mathrm{~J})$ & -- & $1.09 \pm 0.31(\mathrm{~J})$ & -- & $32.7 \pm 6.4(\mathrm{~J})$ & $1.93 \pm 0.39(\mathrm{~J})$ & $1.15 \pm 0.29(\mathrm{~J})$ & -- \\
\hline
\end{tabular}

aBackground concentration listed in Environmental Monitoring Report for the Proposed Ward Valley California Low-Level Radioactive Waste (LLRW) Facility (Atlan-Tech, 1992)

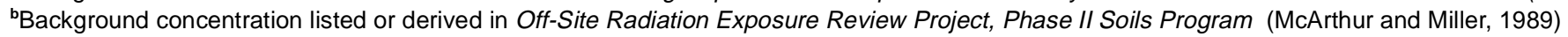

$--=$ Not detected above minimum reporting limit

$\mathrm{J}=$ Estimated value because lab did not provide sample specific MDA bgs = Below ground surface (in feet)

$\mathrm{pCi} / \mathrm{g}=$ Picocuries per gram

$\mathrm{ft}=$ Feet 


\section{A.3.7 Isotope Uranium, Isotopic Plutonium, Isotopic Americium, and Strontium-90 Results}

Isotopic plutonium and strontium-90 were not detected in soil above minimum reporting limits. The isotopic uranium and isotopic americium analytical results for soil samples above minimum reporting limits as specified in the Work Plan for Leachfield CAUs (DOE/NV, 1998), along with the associated background concentrations, are presented in Table A.3-7. The isotopic uranium concentrations listed in the table are in the range found in soil samples taken from undisturbed background locations.

The isotopic americium results in two soil samples (from locations L-2.3 and L-3.1) taken from 0 to $1 \mathrm{ft}$ below the leachfield base (leachrock/soil interface) were elevated in americium-241. The americium-241 concentrations are 2 to 3 times the maximum calculated americium-241 concentrations in soil samples taken from undisturbed background locations (McArthur and Miller, 1989). The maximum calculated dose from the residual americium-241 in the soil results in a dose of $0.02 \mathrm{mrem} / \mathrm{yr}$. This dose rate is about 0.02 percent of the $100 \mathrm{mrem} / \mathrm{yr}$ limit to members of the public established in DOE Order 5400.5 (DOE, 1993). Details on this dose assessment are presented in Attachment 1 of this appendix.

\section{A.3.8 Septic Tank and Distribution Box Sampling Results}

Septic tank and distribution box analytical results exceeding minimum reporting limits are shown in Table A.3-8. The COPCs detected in liquid samples included 1,4-dichlorobenzene, p-isopropyltoluene, trichloroethene, bis(2-ethylhexyl)phthalate, americium-241, uranium-234, and uranium-238.

The COPCs detected in sludge samples from the septic tank and distribution box included americium-241, americium-243, cesium-137, uranium-234, uranium-238, potassium-40, and aroclor-1260. The known uranium source term in Area 25 was the highly enriched uranium-235 fuel used in the ROVER reactors. The concentration of uranium-235 in the water samples is less than the minimum detectable concentrations. However, based on the uranium-234 to uranium-238 ratios, the uranium concentrations demonstrate very low-level contamination from highly enriched uranium, such as that expected from the type of uranium utilized by the NRDS. The PCB, aroclor-1260, was detected at 2,200 $\mu \mathrm{g} / \mathrm{kg}$. Diesel-range organics were detected at 1,400 mg/kg and $78 \mathrm{mg} / \mathrm{kg}$. 
Table A.3-7

Soil Sample Results for Isotopic Americium and Isotopic Uranium, CAU 266 Area 25 Building 3124 Leachfield

\begin{tabular}{|c|c|c|c|c|c|c|c|}
\hline \multirow{3}{*}{$\begin{array}{c}\text { Sample } \\
\text { Location }\end{array}$} & \multirow{2}{*}{$\begin{array}{l}\text { Sample } \\
\text { Number }\end{array}$} & \multirow{2}{*}{$\begin{array}{c}\text { Start Depth } \\
\text { Below } \\
\text { Leachrock } \\
\text { (ft) }\end{array}$} & \multirow{2}{*}{$\begin{array}{c}\text { End Depth } \\
\text { Below } \\
\text { Leachrock } \\
\text { (ft) }\end{array}$} & \multicolumn{4}{|c|}{ Contaminants of Potential Concern $(\mathrm{pCi} / \mathrm{g})$} \\
\hline & & & & Americium-241 & Uranium-234 & Uranium-235 & Uranium-238 \\
\hline & \multicolumn{3}{|c|}{ Background Concentrations } & $0.00006-0.048^{b}$ & $0.1-2.6^{a}$ & $0.5-0.1^{\mathrm{a}}$ & $0.2-3.2^{b}$ \\
\hline \multirow{2}{*}{$\mathrm{L}-1.3$} & TCA60019 & 0 & 1 & -- & $0.85 \pm 0.14$ & $0.07 \pm 0.03(\mathrm{LT})$ & $0.78 \pm 0.13$ \\
\hline & TCA60020 & 2.5 & 3.5 & -- & $1.23 \pm 0.19$ & $0.07 \pm 0.03(\mathrm{LT})$ & $1.21 \pm 0.19$ \\
\hline $\mathrm{L}-1.2$ & TCA60021 & 0 & 1 & -- & $1.02 \pm 0.16$ & -- & $0.87 \pm 0.14$ \\
\hline \multirow{3}{*}{$\mathrm{L}-2.3$} & TCA60025 & 0 & 1 & $0.09 \pm 0.05(\mathrm{LT})$ & $0.96 \pm 0.19$ & -- & $1 \pm 0.19$ \\
\hline & TCA60026 & 2.5 & 3.5 & -- & $1.09 \pm 0.17$ & -- & $1.1 \pm 0.17$ \\
\hline & TCA60027 & 2.5 & 3.5 & -- & $0.95 \pm 0.19$ & -- & $0.96 \pm 0.19$ \\
\hline $\mathrm{L}-2.1$ & TCA60034 & 0 & 1 & -- & $1.04 \pm 0.17$ & -- & $1.01 \pm 0.17$ \\
\hline \multirow{2}{*}{ L-3.1 } & TCA60040 & 0 & 1 & $0.17 \pm 0.05(\mathrm{LT})$ & $0.97 \pm 0.16$ & $0.06 \pm 0.03(\mathrm{LT})$ & $0.89 \pm 0.15$ \\
\hline & TCA60041 & 2.5 & 3.5 & -- & $0.71 \pm 0.17$ & -- & $0.87 \pm 0.19$ \\
\hline \multirow{2}{*}{$\mathrm{L}-4.3$} & TCA60048 & 0 & 1 & -- & $1.06 \pm 0.18$ & $0.07 \pm 0.03(\mathrm{LT})$ & $0.97 \pm 0.16$ \\
\hline & TCA60049 & 0 & 1 & -- & $1.03 \pm 0.17$ & $0.06 \pm 0.03(\mathrm{LT})$ & $0.94 \pm 0.16$ \\
\hline L-4.1 & TCA60053 & 0 & 1 & -- & $0.96 \pm 0.16$ & -- & $1.02 \pm 0.17$ \\
\hline
\end{tabular}

aBackground concentration listed in Environmental Monitoring Report for the Proposed Ward Valley California Low-Level Radioactive Waste (LLRW) Facility (Atlan-Tech, 1992)

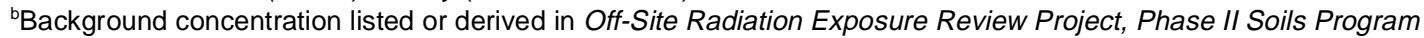
(McArthur and Miller, 1989)

$\mathrm{LT}=$ Concentration is greater than the sample specific minimum detectable concentration but less than the requested minimum detectable concentration

$--=$ Not detected above minimum reporting limit

$\mathrm{pCi} / \mathrm{g}=$ Picocuries per gram

$\mathrm{ft}=$ Feet

Septic tank analytical results for TCLP are shown at the bottom of Table A.3-8. In sample number TCA60002, barium was detected at 934 micrograms per liter $(\mu \mathrm{g} / \mathrm{L})$, cadmium was detected at $53 \mu \mathrm{g} / \mathrm{L}$, and lead was detected at $398 \mu \mathrm{g} / \mathrm{L}$. No other COPCs were detected by TCLP analysis. All detections were less than the regulatory level for the toxicity characteristic (CFR, 1998). 
Table A.3-8

\section{Summary of Septic Tank and Distribution Box Sampling Results Detected Above Minimum Detectable Concentrations, CAU 266 Area 25 Building 3124 Leachfield}

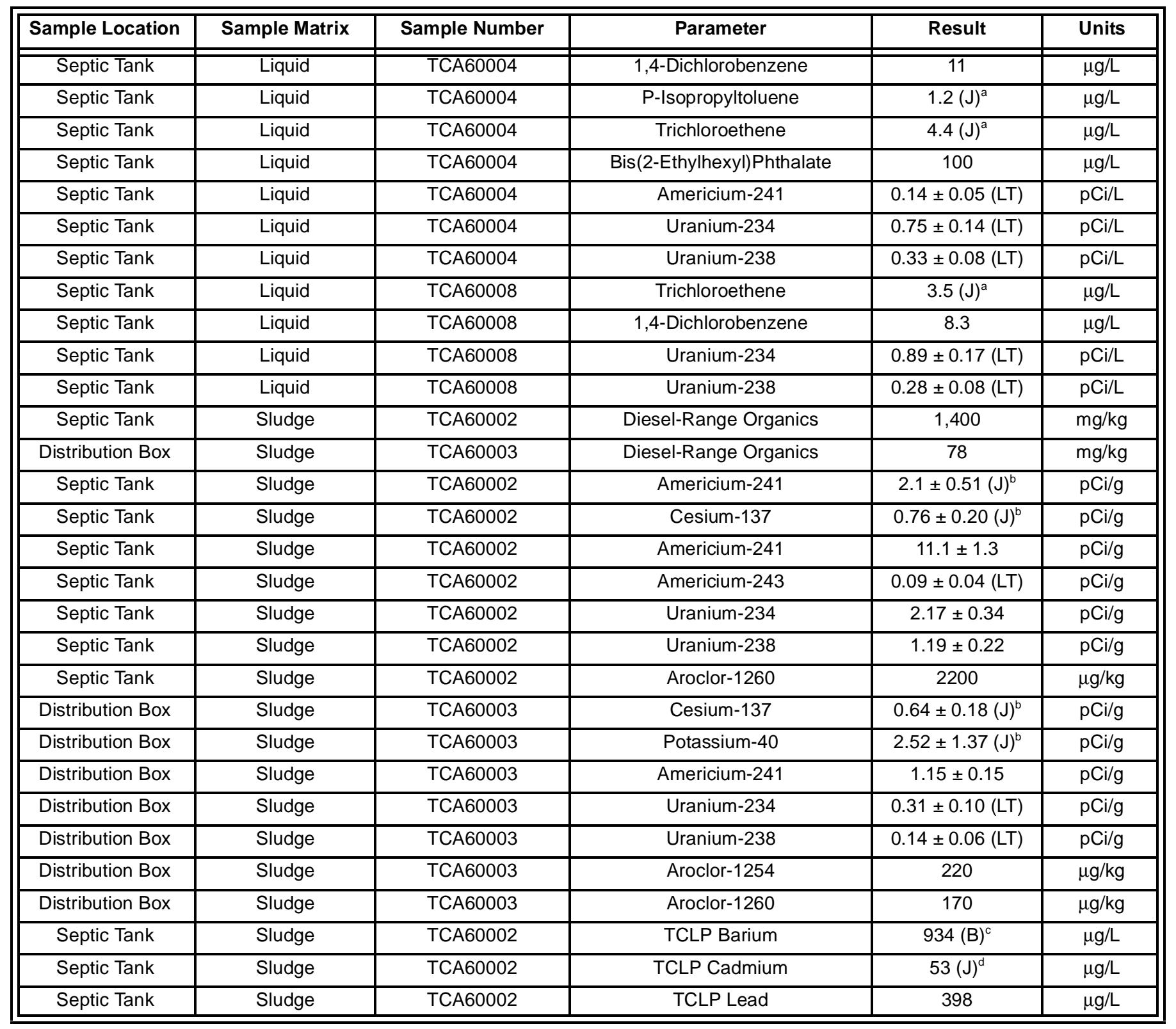

$\mathrm{LT}=$ Concentration greater than sample specific minimum detectable concentration but less than requested minimum detectable concentration

${ }^{a}$ Below minimum reporting limit

${ }^{\mathrm{b}}$ Incomplete initial calibration information and the standard used for what the lab considered to be initial calibration is not gi ven.

${ }^{c}$ Value obtained from a reading that was less than the Contract Required Detection Limit but greater than or equal to the Instrument Detection Limit

${ }^{d}$ Duplicate precision analyses were outside QC limits

$\mathrm{J}=$ Estimated value

$B=$ Estimated value for inorganic analytes

$\mu \mathrm{g} / \mathrm{km}=$ Micrograms per kilogram

$\mathrm{mg} / \mathrm{kg}=$ Milligrams per kilogram

$\mu \mathrm{g} / \mathrm{L}=$ Micrograms per liter

$\mathrm{pCi} / \mathrm{L}=$ Picocuries per liter 


\section{A.4.0 Quality Assurance}

The results of the QA/QC activities for the CAU 266 Area 25 Building 3124 Leachfield corrective action investigation sampling events are summarized in the following text. Detailed information regarding the QA program is contained in the Industrial Sites QAPP (DOE/NV, 1996).

Quality control results are typically judged in terms of precision, accuracy, representativeness, completeness, and comparability and are described in the following sections.

\section{A.4.1 Precision}

Precision is a quantitative measure of the variability of a group of measurements from their average value. Precision is assessed for inorganic analysis by collecting and analyzing duplicate field samples and comparing the results with the original sample. Precision is also assessed by creating, preparing, analyzing, and comparing laboratory duplicates from one or more field samples in inorganic analyses and MS/MSD samples for organic analyses. Precision is reported as relative percent difference (RPD) which is calculated as the difference between the measured concentrations of duplicate samples, divided by the average of the two concentrations, and multiplied by 100. Any deviation from these requirements has been documented and explained and the related data qualified accordingly. The qualification process is described in Section A.4.7.1.

\section{A.4.2 Accuracy}

Analytical accuracy is defined as the nearness of a measurement to the true or accepted reference value. It is the composite of the random and systematic components of the measurement system and measures bias in the measurement system. The random component of accuracy is measured and documented through the analyses of spiked samples. Sampling accuracy is assessed by evaluating the results of spiked samples and laboratory control samples. Accuracy measurements are calculated as percent recovery by dividing the measured sample concentration by the true concentration and multiplying the quotient by 100 .

Field accuracy is assessed by confirming that the documents of record track the sample from origin, through transfer of custody, to disposal. The goal of field accuracy is for all samples to be collected 
from the correct locations at the correct time, placed in a correctly labeled container with the correct preservative, and sealed with custody tape to prevent tampering. All samples in this sampling event were properly collected and forwarded to the laboratories as described above.

\section{A.4.3 Representativeness}

Representativeness expresses the degree to which sample data accurately and precisely represent a characteristic of a population, parameter variations at a sampling point, or an environmental condition (EPA, 1987). Sample representativeness was achieved through the implementation of a sampling program designed to ensure proper sampling locations, number of samples, and the use of validated analytical methods. Representativeness was assessed through analysis of duplicate samples. Representativeness of the samples taken in this sampling event was assured by collecting the specified number of samples (DOE/NV, 1999) and by analyzing them by the approved analytical methods shown in Table A.3-2.

\section{A.4.4 Completeness}

Completeness is defined as a percentage of measurements made that are judged to be valid. A sampling and analytical requirement of 80 percent completeness was established for this project (DOE/NV, 1996b). The minimum 80 percent completeness was achieved although a portion of the acrolein and 2-chloroethyl-vinyl ether results were rejected during data validation.

The specified sampling locations were utilized as planned. All samples were collected as specified in the CAIP (DOE/NV, 1999), and all sample containers reached the laboratory intact and properly preserved (when applicable).

\section{A.4.5 Comparability}

Comparability is a qualitative parameter expressing the confidence with which one data set can be compared to another (EPA, 1987). To ensure comparability, the CAU 266 investigation activities were performed and documented in accordance with approved procedures, and all samples were collected in accordance with the CAIP (DOE/NV, 1999) and the Work Plan for Leachfield CAUs (DOE/NV, 1998). Approved standardized methods and procedures were also used to analyze and report the data (e.g., Contract Laboratory Program [CLP] and/or CLP-like data packages). This 
approach ensures that the data from this project can be compared to other data sets. Based on the minimum comparability requirements specified in the Industrial Sites QAPP (DOE/NV, 1996b), all requirements were met.

Field (i.e., sample-handling) documentation, laboratory nonconformance reports, and the precision and accuracy of quality-control sample results were evaluated for their effect on the results of the associated environmental soil samples. The environmental sample results were then qualified according to processes outlined in the following sections. Documentation of the data qualifications resulting from these reviews is retained in the project files.

\section{A.4.6 Tier I and Tier II Data Evaluations}

All laboratory data from samples collected at CAU 266 have been evaluated for data quality according to the EPA Functional Guidelines (EPA, 1994a and 1994b). These guidelines are implemented in a tiered process and are presented in the following text. No data rejected during the data evaluation process were used to draw the conclusions presented in the CADD/CR. Only valid data, whether estimated (i.e., J-qualified) or not, were used.

Changes resulting from the data evaluation process are documented in project files and are summarized in memoranda for each sample delivery group (SDG). These memoranda are maintained in project files.

\section{A.4.6.1 Tier I Evaluation}

Tier I evaluation for both chemical and radiological analyses examines (but is not limited to):

- Sample count/type consistent with chain of custody

- Analysis count/type consistent with chain of custody

- Correct sample matrix

- Significant problems stated in cover letter or case narrative

- Completeness of certificates of analysis

- Completeness of CLP or CLP-like packages

- Completeness of signatures, dates, and times on chain of custody

- Condition-upon-receipt variance form included

- Requested analyses performed on all samples

- Date received/analyzed given for each sample

- Correct concentration units indicated 
- Electronic data transfer supplied

- Results reported for field and laboratory QC samples

- Whether or not the deliverable met the overall objectives of the project

\section{A.4.6.2 Tier II Evaluation}

Tier II evaluation for both chemical and radiological analyses examines (but is not limited to):

\section{Chemical:}

- Correct detection limits achieved

- Sample date, preparation date, and analysis date for each sample

- Holding time criteria met

- QC batch association for each sample

- Cooler temperature upon receipt

- Sample pH for aqueous samples, as required

- Detection limits properly adjusted for dilution, as required

- Blank contamination evaluated and applied to sample results/qualifiers

- MS/MSD percent recoveries (\%R) and RPDs evaluated and applied to laboratory results/qualifiers

- Field duplicate RPDs evaluated using professional judgement and applied to laboratory results/qualifiers

- Laboratory duplicate RPDs evaluated and applied to laboratory results/qualifiers

- Surrogate \%Rs evaluated and applied to laboratory results/qualifiers

- Laboratory control sample \%R evaluated and applied to laboratory results/qualifiers

- Initial and continuing calibration evaluated and applied to laboratory results/qualifiers

- Internal standard evaluated and applied to laboratory results/qualifiers

- Recalculation of 10 percent of laboratory results from raw data

- Mass spectrometer tuning criteria

- Initial and continuing calibration verification

- Internal standard evaluation

- Organic compound quantitation

- Inductively coupled plasma (ICP) interference check sample evaluation

- Graphite furnace atomic absorption quality control

- ICP serial dilution effects

\section{Radioanalytical:}

- Correct detection limits achieved

- Blank contamination evaluated and applied to sample results/qualifiers

- Certificate of Analysis consistent with data package documentation

- Quality control sample results (duplicates, laboratory control samples, laboratory blanks) evaluated and applied to laboratory result qualifiers

- Sample results, error, and minimum detectable activity evaluated and applied to laboratory result qualifiers 
- Detector system calibrated to National Institute for Standards and Technology (NIST) traceable sources

- Calibration sources preparation was documented, demonstrating proper preparation and appropriateness for sample matrix, emission energies, and concentrations

- Detector system response to daily, weekly, and monthly background and calibration checks for peak energy, peak centroid, peak full-width half-maximum, and peak efficiency

- Tracers NIST-traceable, appropriate for the analysis performed, and recoveries that met QC requirements

- Documentation of all QC sample preparation complete and properly performed

- Spectra lines, emissions, particle energies, peak areas, and background peak areas support the identified radionuclide and its concentration

\section{A.4.6.3 Tier III}

Data quality considerations that are included in EPA data review functional guidelines (EPA, 1994a and 1994b) as a Tier III review include the additional evaluations:

\section{Chemical:}

- Recalculation of all laboratory results from raw data

\section{Radioanalytical:}

- QC sample results (e.g., calibration source concentration, percent recovery, and RPD) verified

- Radionuclides and their concentration appropriate considering their decay schemes, half-lives, and process knowledge and history of the facility and site

- Each identified line in spectra verified against emission libraries and calibration results

- Independent identification of spectra lines, area under the peaks, and quantification of radionuclide concentration in a random number of sample results

A Tier III review of at least five percent of the sample analytical data was performed by Laboratory Data Consultants, Inc. in Carlsbad, California. As a result of the Tier III review, there were no changes to the data contained in the analytical summary tables in Section A.3.0.

\section{A.4.7 Quality Control Samples}

There were 15 trip blanks, 3 field blanks, 4 equipment rinsate blank, 4 MS/MSD, 1 source blank, and 3 field duplicates collected and submitted for laboratory analysis as shown in Table A.3-1. The blanks and duplicates were assigned individual sample numbers and sent to the laboratory "blind." Additional samples were selected by the laboratory to be analyzed as laboratory duplicates. Documentation related to the collection and analyses of these samples is retained in project files. 


\section{A.4.7.1 Field Quality Control Samples}

Review of the field-collected blank analytical data for the investigation sampling indicates that cross-contamination from field methods did not occur during sample collection. Field and equipment rinsate blanks were analyzed for the parameters listed in Table A.3-2 and trip blanks were analyzed for VOCs only. None of the results for these field-collected blanks exceeded the minimum laboratory reporting limits (DOE/NV, 1999).

During the sampling event, three field duplicate soil samples were sent as blind samples to the laboratory to be analyzed for the investigation parameters listed in Table A.3-2. For these samples, the duplicate results precision (i.e., RPDs between the environmental sample results and their corresponding field duplicate sample results) were evaluated to the guidelines set forth in EPA Functional Guidelines (EPA, 1994a and 1994b). The EPA Functional Guidelines state that there are no required review criteria for field duplicate analyses comparability, but allow the data reviewer to exercise professional judgement. The RPD between the environmental samples results and their corresponding field duplicate sample results exceeded the 20 percent criteria stated in the Industrial Sites QAPP (DOE/NV, 1996b) for some target analytes. The variability in the results between the environmental samples and their corresponding field duplicate samples could be attributed to nonhomogeneous samples and the difficulties associated with collecting identical soil field samples. It is expected that soil field duplicate results will have a greater variance than water matrices.

The laboratory duplicate samples were compared to the criteria set forth in the EPA Functional Guidelines (EPA, 1994a) and the associated sample results were qualified accordingly. Both detections and nondetections have been qualified as estimated ( $\mathrm{J}$ and $\mathrm{UJ}$, respectively) if the relative percent difference between an environmental sample and its laboratory duplicate fell outside established criteria.

Four field samples were selected for use as MS/MSD samples. The percent recoveries of these samples (a measure of accuracy) and the relative percent differences in these sample results (a measure of precision) were compared to EPA Functional Guideline criteria (EPA, 1994a and 1994b). The results were used to qualify associated environmental sample results accordingly. 
The EPA Functional Guidelines for review of organic data state that no data qualification action is taken on the basis of MS/MSD results alone. The data reviewer exercises professional judgement in considering these results in conjunction with the results of laboratory control samples (LCSs) and other QC criteria in applying qualifications to the data.

The inorganic data review in EPA Functional Guidelines allows professional judgement to be applied in evaluating the results of matrix spikes (EPA, 1994a). Generally, if the spike recovery is greater than the upper acceptance limits $(>125 \%)$, nondetections are acceptable for use. If the spike recovery is greater than the upper acceptance limits $(>125 \%)$ or less than the lower acceptance limits $(<75 \%)$, positive results are qualified as estimated (J). If spike recovery falls within the range of 30-74\%, nondetections are qualified as estimated (UJ).

\section{A.4.7.2 Laboratory Quality Control Samples}

Analysis of method QC blanks and surrogate spikes for organic analyses, method blanks, preparation blanks, initial and continuing calibration blanks for total metals, and LCS were performed for each SDG by Paragon Analytics, Inc. The results of these analyses were used to qualify associated environmental sample results according to EPA Functional Guidelines (EPA, 1994a and 1994b).

The EPA Functional Guidelines (EPA, 1994a and 1994b) state that no qualification action is taken if a compound is found in an associated blank, but not in the sample or if a compound is found in the sample, but not in an associated blank. The action taken when a compound is detected in both the sample and the associated blank varies depending upon the analyte involved and is described in the "The 5X/10X Rule."

For most VOCs, SVOCs, herbicides, TPH as diesel and gasoline, PCBs, and pesticides, if an analyte is detected in the sample and was also detected in an associated blank, the result is qualified as undetected $(\mathrm{U})$ if the sample concentration is less than five times $(5 \mathrm{X})$ the blank concentration.

For the common laboratory contaminants (e.g., methylene chloride, acetone, 2-butanone [methylethyl ketone or MEK], and phthalate esters [especially bis(2-ethylhexyl)phthalate]), the factor is raised to ten times $(10 \mathrm{X})$ the blank concentration. The sample result is elevated to the quantitation limit if it is 
less than the quantitation limit or remains unaltered if the sample result is greater than or equal to the quantitation limit.

For inorganics (i.e., metals), sample results greater than the instrument detection limit, but less than five times $(5 \mathrm{X})$ the amount found in an associated blank, are qualified as undetected $(\mathrm{U})$. There are no metallic common laboratory contaminants, so there is no "10X Rule" for metals, and the sample result is never altered. When applying the $5 \mathrm{X}$ criteria to soil sample data or calibration blank data, the raw data results are used to evaluate and qualify the reported results on the Certificate of Analysis.

Surrogate spikes, or system monitoring compounds, are added to the environmental samples analyzed by chromatographic techniques for VOCs, SVOCs, herbicides, TPH as diesel and gasoline, PCBs, and pesticides. Surrogate compounds are analytes that are not expected to be present in associated environmental samples, but behave the same as similar target compounds chromatographically. Known amounts of each surrogate are added prior to sample preparation and are carried throughout the preparation/analysis procedure. The percent recoveries of these surrogate compounds give some measure of the anticipated recoveries of the target compounds whose chromatographic behavior they mimic.

If any surrogate percent recoveries are out of the acceptable range (which differs for each surrogate in each method), laboratory protocol calls for the sample to be reprepared and/or reanalyzed. When the surrogate recoveries are acceptable on the second run, only the second analysis results are reported. When both analyses yield the same unacceptable range, the results of both analyses are reported.

The evaluation of surrogate spike percent recovery results is not straightforward. The functional guidelines suggest several optional approaches, but require the data reviewer to exercise professional judgement in reviewing surrogate data and qualifying associated data as estimated ( $\mathrm{J}$ or $\mathrm{UJ}$, for detections or nondetections, respectively) or unusable (R). Documentation of data qualifications resulting from the application of these guidelines is retained in the project files as both hard copy and electronic media.

One laboratory duplicate analysis for metals was performed for each SDG that reported total metals. The duplicate results are compared to the results of the original sample to give a measure of analytical laboratory precision. If the results from a duplicate analysis for a particular analyte fall outside the 
control limits, the EPA Functional Guidelines for Inorganic Data Review (EPA, 1994a) call for all results for that analyte in all associated samples of the same matrix to be qualified as estimated (J). Documentation of data qualifications resulting from the application of these guidelines is retained in the project files as both hard copy and electronic media.

Laboratory control samples, also known as blank spikes, consist of known quantities of target compounds added to purified sand or deionized, distilled water and analyzed along with the environmental samples in the sample delivery group. The percent recoveries of the compounds in the LCS give a measure of laboratory accuracy. The functional guidelines call for the data reviewer to use professional judgement to qualify associated data according to established criteria. Documentation of data qualifications resulting from the application of these guidelines is retained in project files as both hard copy and electronic media.

\section{A.4.8 Field Nonconformances}

During the corrective action investigation, one QA surveillance was conducted by IT Corporation to verify that sampling activities were performed in accordance with applicable requirements. The surveillance did not identify any findings, deficiencies, or nonconformances with sampling activities. The requirements of the plans and procedures governing the activities at the site were met.

\section{A.4.9 Laboratory Nonconformances}

Laboratory nonconformances are generally due to inconsistencies in analytical instrumentation operation, sample preparations, extractions, and fluctuations in internal standard and calibration results. Several laboratory nonconformances were documented for this project. These nonconformances have been accounted for in the data qualification process. All nondetect acrolein and 2-chloroethyl-vinyl ether results were rejected due to the compounds response in the initial calibration. The laboratory is not required to generate a nonconformance for this type of deficiency as long as the laboratory met all the required QC criteria for the initial calibration analysis. Documentation of these results is retained in project files. 


\section{A.5.0 Summary}

Analysis of the data generated from corrective action investigation activities conducted at CAU 266, Area 25 Building 3124 Leachfield, indicates the following:

- The PALs were not exceeded in soil samples for total VOCs, total SVOCs, TPH, total PCBs, total RCRA metals, gamma-emitting radionuclides, isotopic uranium, isotopic plutonium, and strontium-90 for any of the samples collected from the site.

- The isotopic americium (americium-241) concentrations in two soil samples exceed PALs; therefore, americium-241 is a COC at CAU 266. The calculated dose from the residual americium-241 in the soil results is less than the $100 \mathrm{mrem} / \mathrm{yr}$ limit established by DOE Order 5400.5 (DOE, 1993).

- Analytical results from liquid samples collected from the septic tank indicated detection of COPCs to include 1,4-dichlorobenzene, $\mathrm{p}$-isopropyltoluene, trichloroethene, bis(2-ethylhexyl)phthalate, americium-241, uranium-234, and uranium-238. There were no toxicity characteristic chemical COPCs detected above the regulatory levels. Uranium isotopes were detected in ratios that were determined to constitute "added radioactivity."

- Analytical results from sludge samples collected from the septic tank and distribution box indicated detection of COPCs to include americium-241, americium-243, cesium-137, uranium-234, uranium-238, and potassium-40. Americium-241 was detected at levels which were determined to constitute "added radioactivity." Aroclor-1260 was detected at $2,200 \mu \mathrm{g} / \mathrm{kg}$ which is below the regulatory level of $50 \mathrm{mg} / \mathrm{kg}$. Diesel-range organics were detected in the septic tank sludge at $1,400 \mathrm{mg} / \mathrm{kg}$, which exceeds the Nevada regulatory level of $100 \mathrm{mg} / \mathrm{kg}$, and in the distribution box at $78 \mathrm{mg} / \mathrm{kg}$.

- Analytical results from sludge samples collected from the septic tank and distribution box and processed using the TCLP method indicated detection of COPCs to include barium, cadmium, and lead. None of these COPCs were detected above the toxicity characteristic regulatory levels.

- The geologic conditions revealed that the soil within the leachfield is comprised of alluvial fan soil that is poorly sorted with abundant gravel and cobbles. Thin lenses of sand and silt were present at some locations. The soil beneath the leachrock was a poorly sorted, silty gravelly sand with abundant pebbles, some gravel, and a few cobbles.

- The geotechnical and bioassessment samples will not be submitted for analysis because the results will not significantly benefit the corrective action decision process. 


\section{A.6.0 References}

Atlan-Tech Inc. 1992. Environmental Monitoring Report for the Proposed Ward Valley California Low Level Radioactive Waste (LLRW) Facility. Rosewell, GA.

BN, see Bechtel Nevada.

Bechtel Nevada. 1998. Bechtel Nevada Analytical Services Laboratory Procedures Manual. Las Vegas, NV.

Code of Federal Regulations. 1998. Title 40 CFR Parts 261.24, "Toxicity Characteristic." Washington, DC: U.S. Government Printing Office.

DOE, see U.S. Department of Energy.

DOE/NV, see U.S. Department of Energy, Nevada Operations Office.

EC, see Environment Canada.

Environment Canada, Commercial Chemicals Evaluation Branch. 1999. COMMERCIAL CHEMICALS Website, "Acrolein." Hull, Quebec: available at http://24.112.94.181/cceb1/eng/substances/acrolein.htm as accessed on July 16, 1999.

EPA, see U.S. Environmental Protection Agency.

FFACO, see Federal Facility Agreement and Consent Order.

Federal Facility Agreement and Consent Order. 1996 (as amended). Agreed to by the State of Nevada, the U.S. Department of Energy, and the U.S. Department of Defense.

Horwitz, E.P., M.L. Dietz, R. Chiarizia, H. Diamond, A.M. Essling, and D. Graczyk. 1992. Separation and Preconcentration of Uranium from Acidic Media by Extraction Chromatography. In Analytica Chimica Acta, 266: 25-37.

Horwitz, E.P., R. Chiarizia, M.L. Dietz, H. Diamond, D.M. Nelson. 1993. Separation and Preconcentration of Actinides from Acidic Media by Extraction Chromatography. In Analytica Chimica Acta, 281: 361-372.

IT, see IT Corporation.

IT Corporation. 1999. Site-Specific Health and Safety Plan, Septic Waste Systems Investigation, CAUs, 261, 266, and 500, Nevada Test Site, Nevada, Rev. 1. Las Vegas, NV. 
Laczniak, R.J., J.C. Cole, D.A. Sawyer, and D.T. Trudeau. 1996. Summary of Hydrogeological Controls on Ground-Water Flow at the Nevada Test Site, Nye County, Nevada, U.S. Geological Survey Water-Resources Investigations Report 96-4109. Denver, CO: U.S. Geological Survey.

McArthur, R.D., and F.L. Miller, Jr. 1989. Off-Site Radiation Exposure Review Project, Phase II Soil Program, DOE/NV/10384--23. Las Vegas, NV: Desert Research Institute.

Moore, J. 1999. Memorandum to M. Todd (SAIC), entitled "Background Concentrations for NTS and TTR Soil Samples," 3 February. Las Vegas, NV: IT Corporation.

NBMG, see Nevada Bureau of Mines and Geology.

Nevada Bureau of Mines and Geology. 1998. Mineral and Energy Resource Assessment of the Nellis Air Force Range, Open-File Report 98-1. Reno, NV.

Paragon Analytics, Inc. 1998. Standard Operating Procedures Manual, April. Fort Collins, CO.

SNPO, see Space Nuclear Propulsion Office.

Space Nuclear Propulsion Office. 1970. NRDS Master Plan 1969-1970, Nuclear Rocket

Development Station, Jackass Flats, Nevada. Las Vegas, NV: U.S. Atomic Energy Commission.

Spectrum Laboratories, Chemical Fact Sheet. 1999. Spectrum Laboratories Website, "2-chloroethyl-vinyl ether." available at http://www.speclab.com/compound/c110758.htm, as accessed on September 15, 1999.

U.S. Department of Energy. 1988. Site Characterization Plan, Yucca Mountain Site, Nevada, Vols. I-IX, DOE/RW - 0199. Las Vegas, NV.

U.S. Department of Energy. 1993. Radiation Protection of the Public and the Environment, DOE Order 5400.5. Washington, DC.

U.S. Department of Energy, Nevada Operations Office. 1996a. Final Environmental Impact Statement for the Nevada Test Site and Off-Site Locations in the State of Nevada, DOE/EIS 0243. Las Vegas, NV.

U.S. Department of Energy, Nevada Operations Office. 1996b. Industrial Sites Quality Assurance Project Plan, Nevada Test Site, Nevada, Rev. 1, DOE/NV--372. Las Vegas, NV.

U.S. Department of Energy, Nevada Operations Office. 1998. Work Plan for Leachfield Corrective Action Units: Nevada Test Site and Tonopah Test Range, Rev. 1, DOE/NV--514. Las Vegas, NV. 
U.S. Department of Energy, Nevada Operations Office. 1999. Corrective Action Investigation Plan for Corrective Action Unit 266: Area 25 Building 3124 Leachfield, Nevada Test Site, Nevada, DOE/NV--529, Rev. 1. Las Vegas, NV.

U.S. Environmental Protection Agency. 1987. Data Quality Objectives for Remedial Response Activities, EPA/540/G-87-003. Washington, DC.

U.S. Environmental Protection Agency. 1989. AQUIRE Database, ERL-Duluth, U.S. EPA, Phytotox and Terretox databases. Available at http://mail.odsnet.com/TRIFacts/27.html as accessed on September 15, 1999.

U.S. Environmental Protection Agency. 1994a. Contract Laboratory Program National Functional Guidelines for Inorganic Data Review, EPA 540/R-94/013. Washington, DC.

U.S. Environmental Protection Agency. 1994b. Contract Laboratory Program National Functional Guidelines for Organic Data Review, EPA 540/R-94/012. Washington, DC.

U.S. Environmental Protection Agency. 1994c. Guidance for the Data Quality Objectives Process, EPA QA/G-4. Washington, DC.

U.S. Environmental Protection Agency. 1996. Test Methods for Evaluating Solid Waste, Physical/Chemical Methods, SW-846, CD ROM PB97-5019286GEI (CD ROM contains updates for 1986, 1992, 1994 and 1996). Washington, DC.

U.S. Environmental Protection Agency. 1998. Memo from S.J. Smucker to PRG Table Mailing List regarding Region 9 Preliminary Remediation Goals (PRGs), 1 August. San Francisco, CA.

USGS, see U.S. Geological Survey.

U.S. Geological Survey. 1993. Selected Ground-Water Data for Yucca Mountain Region, Southern Nevada and Eastern California, Calendar Year 1993, Open-File Report 95-158. 


\title{
Attachment 1
}

\author{
Memo from S. Adams to R. McCall entitled, \\ "Dose Assessments of the Americium-241 in \\ Soil at the Area 25 Building 3124 Leachfield."
}


DATE: $\quad$ September 15, 1999

TO: $\quad$ Robert McCall

FROM: $\quad$ Steve Adams

SUBJECT: DOSE ASSESSMENT OF THE AMERICIUM-241 IN SOIL AT THE AREA 25 BUILDING 3124 LEACHFIELD

\section{Summary}

Three very conservative assessments were performed to calculate the dose and risk from the residual americium-241 $\left({ }^{241} \mathrm{Am}\right)$ contamination in the Corrective Action Unit (CAU) 266 leachfield system soil. The maximum calculated doses and risks are orders of magnitude less than the criteria established by the U.S. Department of Energy (DOE) and the U.S. Environmental Protection Agency (EPA) for requiring remediation. Leaving the residual contamination in the CAU 266 leachfield system soil would be protective of the public, workers, and the environment.

Americium-241 was detected above background concentrations in soil samples collected at depths greater than 1.067 meters $(\mathrm{m})$ (3.5 feet [ft]) below the ground surface (bgs) at the Area 25 Building 3124 Leachfield, CAU 266. The potential radiological dose and risk from the residual ${ }^{241} \mathrm{Am}$ were calculated by assuming three hypothetical exposure scenarios:

- Exposure Scenario 1 assumes a dose receptor resides on the CAU 266 leachfield full time with the contamination at the current depth.

- Exposure Scenario 2 assumes a dose receptor resides on the CAU 266 leachfield full time subsequent to the ${ }^{241} \mathrm{Am}$ contaminated soil being brought to the surface and mixed with the uncontaminated soil.

- Exposure Scenario 3 assumes a dose receptor resides on the CAU 266 leachfield full time and with the contamination at the ground surface.

Table 1 shows the calculated dose from the residual ${ }^{241}$ Am contamination to a hypothetical future receptor living on the CAU 266 leachfield under the three exposure scenarios analyzed. 
Table 1

Summary of Dose and Risk from Residual ${ }^{241} \mathrm{Am}$ in Soil at CAU 266

\begin{tabular}{|c|c|c||}
\hline \multirow{2}{*}{$\begin{array}{c}\text { Exposure } \\
\text { Scenario } \\
\text { No. }\end{array}$} & \multicolumn{2}{|c|}{ Committed Effective Dose Equivalent and Risk from all Exposure Pathways } \\
\cline { 2 - 3 } & $\begin{array}{c}\text { Dose } \\
\text { (millirem per year) }\end{array}$ & $\begin{array}{c}\text { Risk } \\
\text { (probability of cancer mortality/lifetime) }\end{array}$ \\
\hline \hline 1 & $2.92 \mathrm{E}-18$ & $1.32 \mathrm{E}-24$ \\
\hline 2 & $4.70 \mathrm{E}-3$ & $1.21 \mathrm{E}-9$ \\
\hline 3 & $1.63 \mathrm{E}-2$ & $4.20 \mathrm{E}-9$ \\
\hline
\end{tabular}

The maximum calculated dose is 0.02 percent of the 100 millirem per year (mrem/yr) limit to members of the public established in DOE Order 5400.5 (DOE, 1993). This dose is 0.7 percent of the $25 \mathrm{mrem} / \mathrm{yr}$ dose criterion established by the U.S. Nuclear Regulatory Commission (NRC) for license termination and adopted as a remediation goal by the DOE Nevada Operations Soils Media Project and the Nevada Division of Environmental Protection (NRC, 1997).

The calculated cancer mortality risk from a dose of $1.6 \times 10^{-2} \mathrm{mrem} / \mathrm{yr}$ is $4.2 \times 10^{-9}$. This risk is for Exposure Scenario 3, the scenario with the largest Committed Effective Dose Equivalent (CEDE) and risk. In addition, this level of risk is less than the $1.0 \times 10^{-6}$ to $1.0 \times 10^{-4}$ risk criterion range recommended by the EPA for considering remedial action at Superfund sites (EPA, 1997).

Additional details on the distribution of the ${ }^{241} \mathrm{Am}$ concentration in CAU 266 soil, dose calculation methodology, and risk calculations will be found in the following sections of this memorandum.

\section{Introduction}

The CAU 266 leachfield system received sanitary waste from Building 3124 in Area 25 of the Nevada Test Site. Building 3124 was constructed in 1962 to support the Test Cell A reactor test facility at the Nuclear Rocket Development Station (NRDS). Building 3124 contained equipment for water flow testing, gas flow testing, static pressure testing, equipment maintenance and cleaning, and limited analytical work (DOE/NV, 1999). Subsequent to the deactivation of Test Cell A in 1966, Building 3124 was probably used to house various laboratories in support of the Nevada Applied Ecology Group (DOE/NV, 1999). The building was refurbished in 1991 and used to study radioactively contaminated soil remediation techniques for 2.5 years. After 1995, Building 3124 was used for bench scale testing of soil treatability tests. Building 3124 activities resulted in the release of sanitary waste to the CAU 266 leachfield. Activities conducted within Building 3124 may have introduced radiological material into the CAU 266 leachfield.

Surface and below ground surface soil samples were collected from CAU 266 in 1999. The soil samples were analyzed by gamma spectrometry; alpha spectrometry for isotopic plutonium, uranium, and americium; and for strontium-90. Two samples had analytical results that exceeded background concentrations (McArthur and Miller, 1989). Soil sample TCA60025 was collected from a borehole 
taken from a depth of 1.067 to $1.37 \mathrm{~m}$ (3.5 to $4.5 \mathrm{ft}$ ) below the ground surface. It had a ${ }^{241} \mathrm{Am}$ concentration of 0.092 picocuries per gram $(\mathrm{pCi} / \mathrm{g}) \pm 0.047 \mathrm{pCi} / \mathrm{g}$. Soil sample TCA60040 was collected from a borehole core taken from a depth of four to five feet and had a ${ }^{241} \mathrm{Am}$ concentration of $0.169 \mathrm{pCi} / \mathrm{g} \pm 0.048 \mathrm{pCi} / \mathrm{g}$. The maximum ${ }^{241} \mathrm{Am}$ concentration found in surface soil samples collected from undisturbed background locations, based upon ${ }^{239 / 240} \mathrm{Pu}$ concentrations, is $0.038 \mathrm{pCi} / \mathrm{g}$ (McArthur and Miller, 1989). The maximum ${ }^{241}$ Am concentrations found in the CAU 266 soil are 2.4 times and 4.4 times the background ${ }^{241} \mathrm{Am}$ concentration. The following section of this memorandum discusses the calculational methodologies used to assesses the dose and risk from the residual ${ }^{241} \mathrm{Am}$ in the CAU 266 leachfield system soil.

\section{Dose Methodology}

The residual ${ }^{241} \mathrm{Am}$ concentration in CAU 266 leachfield system soil is only a few times background. The contamination is more than a meter below the ground surface and would be expected to result in a minimal dose and risk to a future land user. The probability is very low that a future land user would receive a significant intake or external exposure to the ${ }^{241} \mathrm{Am}$ in the CAU 266 soil.

Nevertheless, assessments were performed to demonstrate that the dose and risk from the residual ${ }^{241}$ Am contamination are well below regulatory limits. The assessment methodology used to analyze the dose and risk from the residual ${ }^{241}$ Am contamination in the CAU 266 leachfield system soil should not be presumed to set precedence on the methods used in future assessments in support of corrective action levels for DOE Nevada Operations Environmental Remediation Programs. The dose and risk assessment method used for the CAU 266 leachfield system is extraordinarily and simple. The assessment methodology will result in calculated doses and risks that are significantly greater than expected in any realistic future land use at CAU 266. The assessment methodology was selected because the dose and risk from the residual ${ }^{241} \mathrm{Am}$ contamination are recognized intuitively as being very small. In addition, the use of this simple yet conservative methodology will reduce the resource and time required to perform the assessment.

The CAU 266 assessments consist of five components: the exposure scenario, the radiological source terms, the dose assessment calculation methodology, the risk assessment calculation methodology, and the analytical results. Each of the dose assessment components are discussed in the following parts of this memorandum.

\section{Exposure Scenario}

For each of the three exposure scenarios described above, the hypothetical dose receptor is assumed to live continuously at the CAU 266 leachfield. They spend 100 percent of their time outdoors, 24 hours per day, 365 days per year. The only differences in Exposure Scenarios 1, 2, and 3 is the depth and concentration of the radiological source term. For each Exposure Scenario the dose receptor is exposed to ${ }^{241} \mathrm{Am}$ through four exposure pathways. The four exposure pathways include the inhalation of resuspended contaminated soil, inadvertent ingestion of soil, the ingestion of food grown on the CAU 266 leachfield system, and external exposure. The breathing rates, food ingestion rates, annual average mass loading of resuspended soil, fraction of ${ }^{241} \mathrm{Am}$ transferred to vegetation, 
particle size distribution, shielding factors, dose conversion factors, risk factors, unit conversions, and other calculational parameter values are listed in Table A.1.

\section{Radiological Source Term}

Two out of ten CAU 266 soil samples analyzed for isotopic americium had concentrations exceeding that found in soil samples collected from undisturbed background locations

(McArthur and Miller, 1989). Sample TCA60025 was collected from a borehole taken from a depth of $1.067 \mathrm{~m}$ to $1.37 \mathrm{~m}$ ( 3.5 to $4.5 \mathrm{ft}$ ) below the ground surface. It had a ${ }^{241} \mathrm{Am}$ concentration of 0.092 picocuries per gram $(\mathrm{pCi} / \mathrm{g}) \pm 0.047 \mathrm{pCi} / \mathrm{g}$. Soil sample TCA60040 was collected from a borehole core taken from a depth of $1.22 \mathrm{~m}$ to $1.5 \mathrm{~m}$ ( 4 to $5 \mathrm{ft}$ ) and had a ${ }^{241} \mathrm{Am}$ concentration of $0.169 \mathrm{pCi} / \mathrm{g} \pm 0.048 \mathrm{pCi} / \mathrm{g}$. These concentrations are respectively, 2.4 times and 4.4 times the calculated ${ }^{241} \mathrm{Am}$ concentration found in surface soil samples taken from undisturbed background locations (McArthur and Miller, 1989). The radiological source term for Exposure Scenario 1, 2, and 3 are summarized in Table 2 and discussed in detail in the following text.

Table 2

Radiological Source Terms Used in the Assessments of CAU 266

\begin{tabular}{|c|c|c||}
\hline $\begin{array}{c}\text { Exposure } \\
\text { Scenario }\end{array}$ & $\begin{array}{c}\text { Depth of }{ }^{241} \mathbf{A m} \text { Contamination } \\
\text { (mbgs) }\end{array}$ & $\begin{array}{c}{ }^{241} \text { Am Concentration } \\
\text { (pCi/g) }\end{array}$ \\
\hline \hline & Surface to $1.067 /$ & 0.0 \\
1 & 1.067 to 1.22 & 0.092 \\
& 1.22 to 1.5 & 0.169 \\
& $>1.5$ & 0.0 \\
\hline \multirow{2}{*}{2} & 0 to 1.5 & 0.0409 \\
& $>1.5$ & 0.0 \\
\hline \multirow{2}{*}{3} & 0 to 0.433 & 0.14 \\
& $>0.43$ & 0.0 \\
\hline
\end{tabular}

In Exposure Scenario 1 doses and risks were calculated assuming that the CAU 266 leachfield system soil is not contaminated to a depth of $1.067 \mathrm{~m}(3.5 \mathrm{ft}) \mathrm{bgs}$. From a depth of $1.067 \mathrm{~m}(3.5 \mathrm{ft}) \mathrm{bgs}$ to a

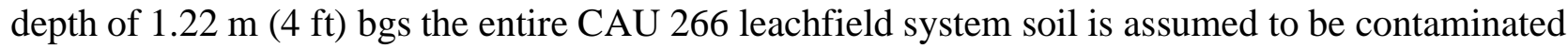

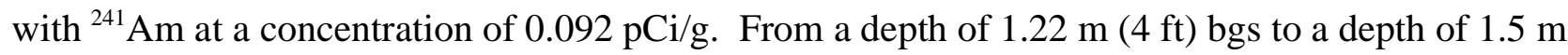
( $5 \mathrm{ft}$ ) bgs the entire CAU 266 leachfield system soil is assumed to be contaminated with ${ }^{241} \mathrm{Am}$ at a concentration of $0.169 \mathrm{pCi} / \mathrm{g}$. This radiological source term should conservatively bound the true ${ }^{241}$ Am concentration in soil at the CAU 266 leachfield.

For Exposure Scenario 2 the ${ }^{241}$ Am contaminated soil was assumed to be uniformly mixed with the clean soil located between the contaminated soil and the ground surface. Mixing the contaminated soil with the clean soil would result in a ${ }^{241} \mathrm{Am}$ concentration of $0.0409 \mathrm{pCi} / \mathrm{g}$ in all soil located in the CAU 266 leachfield system from the ground surface to a depth of $1.5 \mathrm{~m}(5 \mathrm{ft})$. This value was calculated in the following manner: 
${ }^{241} \mathrm{Am}$ concentration $=[0.092 \mathrm{pCi} / \mathrm{g} \times(1.22 \mathrm{~m}-1.067 \mathrm{~m})]+[0.169 \mathrm{pCi} / \mathrm{g} \times(1.5 \mathrm{~m}-1.22 \mathrm{~m})] /(1.067 \mathrm{~m}+0.153 \mathrm{~m}+0.28 \mathrm{~m})$

${ }^{241} \mathrm{Am}$ concentration $=0.0409 \mathrm{pCi} / \mathrm{g}$

The ${ }^{241} \mathrm{Am}$ contaminated soil at the surface would increase the dose and risk per unit concentration of ${ }^{241} \mathrm{Am}$. However, mixing the ${ }^{241} \mathrm{Am}$ contaminated soil with clean soil also dilutes the ${ }^{241} \mathrm{Am}$ concentration and thereby reduce the calculated dose and risk.

In Exposure Scenario 3 the contaminated soil is assumed to extend from the ground surface to a depth of $0.433 \mathrm{~m}(1.42 \mathrm{ft})$. The dose receptor is assumed to be living directly on the residual ${ }^{241} \mathrm{Am}$ contaminated soil.

\section{Dose and Risk Calculation Methodology}

Simple, conservative, dose and risk calculation models were used to calculate the CEDE and cancer mortality risk to the hypothetical future land user at CAU 266. The calculational dose models for inhalation, food ingestion, soil ingestion, and external exposure are described below. The CEDE is calculated by summing the dose contribution from each of the four exposure pathways. The ${ }^{241} \mathrm{Am}$ risk models are from the EPA Federal Guidance Report No. 13 (Eckerman, et al., 1998). The equations used to calculate the ${ }^{241} \mathrm{Am}$ intakes, exposure, and the resultant dose and risks are shown in Attachment A.1. Parameter values used in the equations and references for each parameter value are listed in Table A.1. The dose and risk calculations are shown in Attachment A.2.

\section{$\underline{\text { Analytical Results }}$}

The calculated dose and risk for each exposure scenario from each exposure pathway are shown in Table 3.

Table 3

Calculated Committed Effective Dose Equivalent (mrem/yr) and Risks from Residual 241-Am in CAU 266 Leachfield System Soil

\begin{tabular}{|c|c|c|c|c|c|c|c|c|c|c||}
\hline \multirow{2}{*}{$\begin{array}{c}\text { Exposure } \\
\text { Scenario }\end{array}$} & \multicolumn{2}{|c|}{ Inhalation } & \multicolumn{2}{|c|}{ Food Ingestion } & Soil Ingestion & External Exposure & \multicolumn{3}{|c|}{ Total } \\
\cline { 2 - 13 } & Dose & Risk & Dose & Risk & Dose & Risk & Dose & Risk & Dose & Risk \\
\hline \hline 1 & 0 & 0 & 0 & 0 & 0 & 0 & $2.9 \mathrm{E}-18$ & $1.3 \mathrm{E}-24$ & $2.9 \mathrm{E}-18$ & $1.3 \mathrm{E}-24$ \\
\hline 2 & $3.3 \mathrm{E}-4$ & $1.1 \mathrm{E}-10$ & $2.6 \mathrm{E}-3$ & $3 \mathrm{E}-10$ & $1.1 \mathrm{E}-4$ & $1.4 \mathrm{E}-11$ & $1.7 \mathrm{E}-3$ & $7.6 \mathrm{E}-10$ & $4.7 \mathrm{E}-3$ & $1.2 \mathrm{E}-9$ \\
\hline 3 & $1.1 \mathrm{E}-3$ & $3.8 \mathrm{E}-10$ & $9.0 \mathrm{E}-3$ & $1.2 \mathrm{E}-9$ & $3.8 \mathrm{E}-4$ & $4.9 \mathrm{E}-11$ & $5.8 \mathrm{E}-3$ & $2.6 \mathrm{E}-9$ & $1.6 \mathrm{E}-2$ & $4.2 \mathrm{E}-9$ \\
\hline
\end{tabular}

Note - The risk component is expressed as the increase in the probability of cancer mortality to the dose receptor during their lifetime. 
The maximum calculated dose from the residual ${ }^{241} \mathrm{Am}$ contamination at CAU 266 leachfield system is for Exposure Scenario 3 and is equal to $1.63 \times 10^{-2} \mathrm{mrem} / \mathrm{yr}$. About 55 percent of the dose is from food ingestion, 36 percent from external exposure, seven percent from inhalation, and two percent from soil ingestion. The maximum calculated dose is less than 0.2 percent of the remediation criterion established in DOE Order 5400.5 (DOE, 1993). In addition, it is less than 0.7 percent of the remediation criterion established by the NRC for licence termination. The calculated dose for Exposure Scenario 2 is $4.7 \times 10^{-3} \mathrm{mrem} / \mathrm{yr}$. It is less than 24 percent of the calculated dose for Exposure Scenario 3. The calculated dose for Exposure Scenario 1 is $2.92 \times 10^{-18}$, which is insignificant.

The maximum calculated risk from the residual ${ }^{241} \mathrm{Am}$ contamination at CAU 266 leachfield system is for Exposure Scenario 3 and is equal to a $4.2 \times 10^{-9}$ increase in the cancer mortality to the dose receptor during their lifetime. This level of risk does not exceed the EPA recommended level for considering remediation at Superfund sites (EPA, 1997).

\section{Conclusion}

Three very conservative assessments were performed to calculate the dose and risk from the residual ${ }^{241}$ Am contamination in the CAU 266 leachfield system soil. The maximum calculated doses and risks are orders of magnitude less than the criteria established by the DOE and EPA for requiring remediation. Therefore, leaving the residual contamination in the CAU 266 leachfield system soil would be protective of the public, workers, and the environment.

$\begin{array}{llll}\text { cc: } & \text { Mike Foley } & \text { Charles Orchard } & \text { Dustin Wilson } \\ \text { Mike O'Hagan } & \text { Stacey Alderson } & \text { Central File } \\ \text { Bruce Dionne } & \text { Carl Speer } & \end{array}$




\section{Attachment A.1}

\section{Calculation Equations}




\section{Inhalation Dose Calculational Model}

$$
\mathrm{H}_{\mathrm{inh}}=\mathrm{C} \times \mathrm{ML} \times \mathrm{BR} \times \mathrm{T} \times \mathrm{DR} \times \mathrm{FD} \quad \text { where: }
$$

$\mathrm{H}_{\text {inh }}=\mathrm{CEDE}$ from inhalation of ${ }^{241} \mathrm{Am}(\mathrm{mrem} / \mathrm{yr})$

$\mathrm{C}=$ Concentration of ${ }^{241} \mathrm{Am}$ in the soil $(\mathrm{pCi} / \mathrm{g})$

$\mathrm{ML}=$ Annual average mass loading of resuspended soil in the air gram per cubic meter $\left(\mathrm{g} / \mathrm{m}^{3}\right)$

$\mathrm{BR}=$ Average daily breathing rate cubic meter per day $\left(\mathrm{m}^{3} /\right.$ day $)$

$\mathrm{T}=$ Exposure time (days)

$\mathrm{DR}=$ Dose conversion factor for inhalation of ${ }^{241} \mathrm{Am}(\mathrm{mrem} / \mathrm{pCi})$

FD = Depth factor, fraction of contaminated soil that will be resuspended

\section{Inhalation Risk Calculational Model}

$$
\mathrm{R}_{\mathrm{inh}}=\mathrm{C} \times \mathrm{ML} \times \mathrm{BR} \times \mathrm{T} \times \mathrm{Rf}_{\mathrm{inh}} \times \mathrm{FD} \quad \text { where: }
$$

$\mathrm{R}_{\mathrm{inh}}=$ Cancer mortality risk from the inhalation of ${ }^{241} \mathrm{Am}$ (lifetime probability)

$\mathrm{C}=$ Concentration of ${ }^{241} \mathrm{Am}$ in the soil ( $\mathrm{pCi} / \mathrm{g}$ )

$\mathrm{ML}=$ Annual average mass loading of resuspended soil in the air $\left(\mathrm{g} / \mathrm{m}^{3}\right)$

$\mathrm{BR}=$ Average daily breathing rate $\left(\mathrm{m}^{3} /\right.$ day $)$

$\mathrm{T}=$ Exposure time (days)

$\mathrm{Rf}_{\text {inh }}=$ Cancer mortality risk factor for inhalation of ${ }^{241} \mathrm{Am}$ (lifetime probability/pCi)

FD = Depth factor, fraction of contaminated soil that will be resuspended

\section{Food Ingestion Dose Calculational Model}

$\mathrm{H}_{\text {ingf }}=\mathrm{C} \times \mathrm{B}_{\mathrm{v}} \times \mathrm{I}_{\mathrm{r}} \times \mathrm{T} \times \mathrm{DCF} \times 3,700 \times 0.25 \times \mathrm{F}_{\mathrm{u}} \quad$ where:

$\mathrm{H}_{\text {ingf }}=\mathrm{CEDE}$ from ingestion of ${ }^{241} \mathrm{Am}$ contaminated food (mrem/yr)

$\mathrm{C}=$ Concentration of ${ }^{241} \mathrm{Am}$ in the soil $(\mathrm{pCi} / \mathrm{g})$

$\mathrm{B}_{\mathrm{v}} \quad=$ Fraction of ${ }^{241} \mathrm{Am}$ transferred from soil to food

$\mathrm{I}_{\mathrm{r}} \quad=$ Ingestion rate of food gram per day $(\mathrm{g} / \mathrm{d})$

$\mathrm{T}=$ Exposure time of individual to food grown on CAU $266(\mathrm{~d} / \mathrm{yr})$

$\mathrm{DCF}=$ Ingestion dose conversion factor for ${ }^{241} \mathrm{Am}$ (sieverts/becquerel $[\mathrm{Sv} / \mathrm{Bq}]$ )

$3,700=$ Multiplication conversion factor $(\mathrm{mrem} / \mathrm{pCi}) /(\mathrm{Sv} / \mathrm{Bq})$

$0.25=$ Area correction factor for CAU 266 (dimensionless)

$\mathrm{F}_{\mathrm{u}} \quad=$ Fraction of ${ }^{241} \mathrm{Am}$ taken up by the roots due to vertical distribution of the ${ }^{241} \mathrm{Am}$ in soil 


\section{Food Ingestion Risk Calculational Model}

$\mathrm{R}_{\text {ing }}=\mathrm{C} \times \mathrm{B}_{\mathrm{v}} \times \mathrm{I}_{\mathrm{r}} \times \mathrm{T} \times \mathrm{Rf}_{\text {ing }} \times 0.25 \times \mathrm{F}_{\mathrm{u}} \quad$ where:

$\mathrm{R}_{\mathrm{ing}}=$ Cancer mortality risk from the ingestion of ${ }^{241} \mathrm{Am}$ contaminated food (lifetime probability)

$\mathrm{C}=$ Concentration of ${ }^{241} \mathrm{Am}$ in the soil (pCi/g)

$\mathrm{B}_{\mathrm{v}} \quad=$ Fraction of ${ }^{241} \mathrm{Am}$ transferred from soil to food

$\mathrm{I}_{\mathrm{r}} \quad=$ Ingestion rate of food $(\mathrm{g} / \mathrm{d})$

$\mathrm{T}=$ Exposure time of individual to food grown on CAU $266(\mathrm{~d} / \mathrm{yr})$

$\mathrm{Rf}_{\text {ing }}=$ Cancer mortality risk factor for ingestion of ${ }^{241} \mathrm{Am}$ (lifetime probability/pCi)

$0.25=$ Area correction factor for CAU 266 (dimensionless)

$\mathrm{F}_{\mathrm{u}} \quad=$ Fraction of ${ }^{241} \mathrm{Am}$ taken up by the roots due to vertical distribution of the ${ }^{241} \mathrm{Am}$ in soil

\section{Soil Ingestion Dose Calculational Model}

$$
\mathrm{H}_{\text {ings }}=\mathrm{C} \times \mathrm{I}_{\mathrm{rs}} \times \mathrm{T} \times \mathrm{DF}_{\mathrm{s}} \times 3,700 \times \mathrm{FD}_{\mathrm{u}} \quad \text { where: }
$$

$\mathrm{H}_{\text {ings }}=$ Dose from the ingestion of ${ }^{241} \mathrm{Am}$ contaminated soil

$\mathrm{C}=$ Concentration of ${ }^{241} \mathrm{Am}$ in the soil $(\mathrm{pCi} / \mathrm{g})$

$\mathrm{I}_{\mathrm{rs}} \quad=$ Ingestion rate of soil $(\mathrm{g} / \mathrm{d})$

$\mathrm{T}=$ Exposure time of individual to soil ingestion on CAU $266(\mathrm{~d} / \mathrm{yr})$

$\mathrm{DF}_{\mathrm{s}}=$ Ingestion dose conversion factor for $\left.{ }^{241} \mathrm{Am}(\mathrm{Sv} / \mathrm{Bq})\right)$

$3,700=$ Multiplication conversion factor $(\mathrm{mrem} / \mathrm{pCi}) /(\mathrm{Sv} / \mathrm{Bq})$

$\mathrm{FD}_{\mathrm{u}}=$ Fraction of ${ }^{241} \mathrm{Am}$ ingested, a function of the vertical distribution of the ${ }^{241} \mathrm{Am}$ in soil

\section{Soil Ingestion Risk Calculational Model}

$$
\mathrm{R}_{\text {ings }}=\mathrm{C} \times \mathrm{I}_{\mathrm{rs}} \times \mathrm{T}_{\mathrm{s}} \times \mathrm{Rf}_{\mathrm{s}} \times \mathrm{FD}_{\mathrm{u}} \quad \text { where: }
$$

$\mathrm{R}_{\text {ings }}=$ Cancer mortality risk from the ingestion of ${ }^{241} \mathrm{Am}$ contaminated soil (lifetime probability)

$\mathrm{C}=$ Concentration of ${ }^{241} \mathrm{Am}$ in the soil $(\mathrm{pCi} / \mathrm{g})$

$\mathrm{I}_{\mathrm{rs}} \quad=$ Ingestion rate of soil $(\mathrm{g} / \mathrm{d})$

$\mathrm{T}_{\mathrm{s}} \quad=$ Exposure time of individual to soil ingestion on CAU $266(\mathrm{~d} / \mathrm{yr})$

$\mathrm{Rf}_{\mathrm{s}} \quad=$ Cancer mortality risk factor for ingestion of ${ }^{241} \mathrm{Am}$ (lifetime probability/pCi)

$\mathrm{FD}_{\mathrm{u}}=$ fraction of ${ }^{241} \mathrm{Am}$ ingested, a function of the vertical distribution of the ${ }^{241} \mathrm{Am}$ in soil 
External Exposure Dose Model

$$
\mathrm{H}_{\mathrm{ext}}=\mathrm{C} \times \mathrm{DF}_{\mathrm{ext}} \times \mathrm{T}_{\mathrm{ext}} \times \mathrm{CF} \times \mathrm{SF} \quad \text { where: }
$$

$\mathrm{H}_{\mathrm{ext}}=$ The external dose from ${ }^{241} \mathrm{Am}$ in the soil (mrem/yr)

$\mathrm{C}=$ Concentration of ${ }^{241} \mathrm{Am}$ in the soil $(\mathrm{pCi} / \mathrm{g})$

$\mathrm{DF}_{\mathrm{ext}}=$ External dose conversion factor for ${ }^{241} \mathrm{Am}(\mathrm{Sv} / \mathrm{Bq}) /\left(\mathrm{s} / \mathrm{m}^{3}\right)$

$\mathrm{T}_{\mathrm{ext}} \quad=$ Exposure time $(\mathrm{s} / \mathrm{yr})$

$\mathrm{CF}=$ Conversion factor $[(\mathrm{mrem} / \mathrm{yr}) /(\mathrm{pCi} / \mathrm{g})] /\left[(\mathrm{Sv} / \mathrm{Bq}) /\left(\mathrm{s} / \mathrm{m}^{3}\right)\right]$

$\mathrm{SF} \quad=$ Shielding factor for ${ }^{241} \mathrm{Am}$ below the surface (dimensionless)

\section{External Exposure Risk Model}

$$
\mathrm{R}_{\mathrm{ext}}=\mathrm{C} \times \mathrm{RF}_{\mathrm{ext}} \times \mathrm{T}_{\mathrm{ext}} \times \mathrm{CF} \times \mathrm{SF} \quad \text { where: }
$$

$\mathrm{R}_{\mathrm{ext}} \quad=$ The external exposure risk from ${ }^{241} \mathrm{Am}$ in the soil (mrem/yr)

$\mathrm{C}=$ Concentration of ${ }^{241} \mathrm{Am}$ in the soil $(\mathrm{pCi} / \mathrm{g})$

$\mathrm{RF}_{\text {ext }}=$ External exposure risk factor for ${ }^{241} \mathrm{Am}(\mathrm{kg} / \mathrm{Bq} / \mathrm{s})$

$\mathrm{T}_{\text {ext }}=$ Exposure time $(\mathrm{s} / \mathrm{yr})$

$\mathrm{CF}=$ Conversion factor $(\mathrm{g}-\mathrm{s}-\mathrm{Bq} / \mathrm{kg}-\mathrm{yr}-\mathrm{pCi})$

$\mathrm{SF} \quad=$ Shielding factor for ${ }^{241} \mathrm{Am}$ below the surface (dimensionless) 
Table A.1

Numerical Values Used in the CAU 266 Dose and Risk Assessments

(Page 1 of 2)

\begin{tabular}{|c|c|c|}
\hline Parameter & Value & Reference \\
\hline $\mathrm{C}_{1 \mathrm{a}}$ & $0.092 \mathrm{pCi} / \mathrm{g}$ from $1.07 \mathrm{~m}$ to $1.22 \mathrm{~m} \mathrm{bgs}$ & CAU 266 Sample TCA60025 (Scenario 1) \\
\hline $\mathrm{C}_{1 \mathrm{~b}}$ & $0.169 \mathrm{pCi} / \mathrm{g}$ from $1.22 \mathrm{~m}$ to $1.5 \mathrm{~m}$ bgs & CAU 266 Sample TCA60040 (Scenario 1) \\
\hline $\mathrm{C}_{2}$ & $0.0409 \mathrm{pCi} / \mathrm{g}$ & Scenario 2 \\
\hline $\mathrm{C}_{3}$ & $\begin{array}{l}0.092 \mathrm{pCi} / \mathrm{g} \text { from } 0 \mathrm{~m} \text { to } 0.153 \mathrm{~m} \text { bgs } \\
0.169 \mathrm{pCi} / \mathrm{g} \text { from } .153 \mathrm{~m} \text { to } .43 \mathrm{~m} \text { bgs }\end{array}$ & Scenario 3 \\
\hline $\mathrm{H}_{\mathrm{inh}}$ & $\mathrm{mrem} / \mathrm{yr}$ & Calculated value \\
\hline $\mathrm{H}_{\text {ingf }}$ & $\mathrm{mrem} / \mathrm{yr}$ & Calculated value \\
\hline $\mathrm{H}_{\text {ings }}$ & $\mathrm{mrem} / \mathrm{yr}$ & Calculated value \\
\hline $\mathrm{H}_{\mathrm{ext}}$ & $\mathrm{mrem} / \mathrm{yr}$ & Calculated value \\
\hline $\mathrm{R}_{\mathrm{inh}}$ & lifetime cancer death probability & Calculated value \\
\hline $\mathrm{R}_{\text {ingf }}$ & lifetime cancer death probability & Calculated value \\
\hline $\mathrm{R}_{\text {ings }}$ & lifetime cancer death probability & Calculated value \\
\hline $\mathrm{R}_{\text {ext }}$ & lifetime cancer death probability & Calculated value \\
\hline $\mathrm{ML}$ & $1.3 \times 10^{-5} \mathrm{~g} / \mathrm{m}^{3}$ & Shinn, 1994 \\
\hline $\mathrm{BR}$ & $16 \mathrm{~m}^{3} / \mathrm{d}$ & Table 5, 19 - 34 year old males (Layton, 1993) \\
\hline $\mathrm{T}$ & $365 \mathrm{~d} / \mathrm{yr}$ & Maximum possible value \\
\hline$T_{\text {ext }}$ & $3.156 \times 10^{7} \mathrm{~s} / \mathrm{yr}$ & Maximum possible value \\
\hline DR & $0.0999 \mathrm{mrem} / \mathrm{pCi}$ & ICRP (1995) for Class M, $5 \mu \mathrm{m}$ AMAD, ${ }^{241} \mathrm{Am}$ \\
\hline DCF & $2.0 \times 10^{-7} \mathrm{~Sv} / \mathrm{Bq}$ & ICRP (1995) for Class M ${ }^{241} \mathrm{Am}$ \\
\hline $\mathrm{DCF}_{\mathrm{s}}$ & $2.0 \times 10^{-7} \mathrm{~Sv} / \mathrm{Bq}$ & ICRP (1995) for Class M ${ }^{241} \mathrm{Am}$ \\
\hline $\mathrm{DF}_{\mathrm{ext}}$ & $2.34 \times 10^{-19}(\mathrm{~Sv} / \mathrm{Bq}) /\left(\mathrm{s} / \mathrm{m}^{3}\right)$ & $\begin{array}{l}\text { Table III.7 Eckerman and Ryman 1993, }{ }^{241} \text { Am, infinite } \\
\text { depth }\end{array}$ \\
\hline $\mathrm{Rf}_{\text {inh }}$ & $9.04 \times 10^{-7} / \mathrm{Bq}$ & Table 2.1, Eckerman et al., $1998,{ }^{241} \mathrm{Am}$, cancer mortality \\
\hline $\mathrm{Rf}_{\text {ing }}$ & $2.56 \times 10^{-9} / \mathrm{Bq}$ & $\begin{array}{l}\text { Table } 2.3 a, \text { Eckerman et al., } 1998,{ }^{241} \mathrm{Am} \text {, cancer } \\
\text { mortality }\end{array}$ \\
\hline $\mathrm{Rf}_{\text {ings }}$ & $2.56 \times 10^{-9} / \mathrm{Bq}$ & $\begin{array}{l}\text { Table } 2.3 a \text {, Eckerman et al., 1998, }{ }^{241} \mathrm{Am} \text {, cancer } \\
\text { mortality }\end{array}$ \\
\hline$R f_{\text {ext }}$ & $1.59 \times 10^{-17} \mathrm{~kg} / \mathrm{Bq}-\mathrm{s}$ & Table 2.4 , Eckerman et al., $1998,{ }^{241} \mathrm{Am}$, cancer mortality \\
\hline FD & $\begin{array}{l}0 \text { for }{ }^{241} \mathrm{Am}>0.15 \mathrm{~m} \text { bgs } \\
1 \text { for }{ }^{241} \mathrm{Am} \leq 0.15 \mathrm{~m} \text { bgs }\end{array}$ & Equations B.5, page 144 Yu et al., 1989 \\
\hline $\mathrm{B}_{\mathrm{v}}$ & $5.39 \times 10^{-4}$ fraction & Calculated, Tables D.2 - D-4, Yu et al., 1989 \\
\hline
\end{tabular}


Table A.1

Numerical Values Used in the CAU 266 Dose and Risk Assessments (Page 2 of 2)

\begin{tabular}{|c|c|c|}
\hline Parameter & Value & Reference \\
\hline $\mathrm{I}_{\mathrm{r}}$ & $2,952 \mathrm{kcal} / \mathrm{d}$ & Eckerman et al., 1998 \\
\hline 3700 & (mrem/pCi)/(Sv/Bq) & Unit definition, Shleien et al., 1998 \\
\hline 0.25 & Dimensionless & Equations D-5, page 179, Yu et al., 1989 \\
\hline $\mathrm{F}_{\mathrm{u}}$ & $\begin{array}{l}0 \text { for initial conditions, } 1 \text { when } \\
{ }^{241} \mathrm{Am} \text { is within root zone depth }\end{array}$ & Equations D.6, page 180, Yu et al., 1989 \\
\hline $\mathrm{I}_{\mathrm{rs}}$ & $0.01 \mathrm{~g} / \mathrm{d}$ & Section 6A.1, for adults, AIHC, 1994 \\
\hline $\mathrm{FD}_{\mathrm{u}}$ & $\begin{array}{l}0 \text { for }{ }^{241} \mathrm{Am}>0.15 \mathrm{~m} \text { bgs } \\
1 \text { for }{ }^{241} \mathrm{Am} \leq 0.15 \mathrm{mbgs}\end{array}$ & Equations B.5, page 144 Yu et al., 1989 \\
\hline $\mathrm{CF}$ & $[(\mathrm{mrem} / \mathrm{yr}) / \mathrm{pCi} / \mathrm{g})] /\left[(\mathrm{Sv} / \mathrm{Bq}) /\left(\mathrm{s} / \mathrm{m}^{3}\right)\right]$ & Unit definition, Shleien et al., 1998 \\
\hline SF & $\begin{array}{l}2.73 \times 10^{-16}(\mathrm{mrem} / \mathrm{yr}) /(\mathrm{pCi} / \mathrm{g}) \text { when } \\
{ }^{241} \mathrm{Am} \geq 1.067 \mathrm{~m} \mathrm{bgs}, \text { otherwise }=1\end{array}$ & $\begin{array}{l}\text { Calculated using RESRAD code, Files RCRSCN17.RAD } \\
\text { and RCRSACN2.RAD }\end{array}$ \\
\hline
\end{tabular}




\section{Attachment A.2}

\section{Dose and Risk Assessment Due to Am-241 in Soil at CAU 266 Leachfield}




\section{Inhalation Exposure Pathway}

Inhalation Dose $=\mathrm{H}=\mathrm{C} \times \mathrm{ML} \times \mathrm{BR} \times \mathrm{T} \times \mathrm{DR} \times \mathrm{FD}$

Inhalation Risk $=\mathrm{R}=\mathrm{C} \times \mathrm{ML} \times \mathrm{BR} \times \mathrm{T} \times \mathrm{Rf} \times \mathrm{FD}$

where

$\mathrm{H}=$ The 50-year dose due to the Am-241 inhaled during the year of the maximum intake (mrem/year)

$\mathrm{R}=$ The cancer mortality risk from inhalation of Am-241

$\mathrm{C}=$ is the concentration of $\mathrm{Am}-241$ in the soil $(\mathrm{pCi} / \mathrm{g})$

$$
\begin{aligned}
& \mathrm{C} 1=0.092 \mathrm{pCi} / \mathrm{g} \text { from } 1.07 \mathrm{~m} \text { to } 1.22 \mathrm{~m} \text { bgs, Exposure Scenario } 1 \\
& \mathrm{C} 1=0.169 \mathrm{pCi} / \mathrm{g} \text { from } 1.22 \mathrm{~m} \text { to } 1.5 \mathrm{~m} \mathrm{bgs} \text {, Exposure Scenario } 1 \\
& \mathrm{C} 2=0.0409 \mathrm{pCi} / \mathrm{g} \text { from } 0 \mathrm{~m} \text { to } 1.5 \mathrm{~m} \mathrm{bgs} \text {, Exposure Scenario } 2 \\
& \mathrm{C} 3=0.092 \mathrm{pCi} / \mathrm{g} \text { from } 0.0 \mathrm{~m} \text { to } 0.153 \mathrm{~m} \mathrm{bgs} \text {, Exposure Scenario } 3 \\
& \mathrm{C} 3=0.169 \mathrm{pCi} / \mathrm{g} \text { from } 0.153 \mathrm{~m} \text { to } 0.43 \mathrm{~m} \text { bgs, Exposure Scenario } 3 \\
& \text { Note }- \text { For calculational purposes the Scenario } 3 \text { source term }= \\
& \text { the weighted average Am-241concentration in the soil from } 0.0 \text { to } 0.43 \mathrm{~m} \text { bgs }
\end{aligned}
$$

$M L=$ the annual average mass loading of resuspended soil in the air $(\mathrm{g} / \mathrm{m} 3)($ Shinn, 1994) =

$\mathrm{T}=$ the exposure time in days per year $(\mathrm{d} / \mathrm{yr})=$

$\mathrm{DR}=$ the dose conversion factor for Am-241 Class M, 5 micorn AMAD, (Sv/Bq), ICRP (1995) Publication \#68 =

Exposure Scenario \#1 =

\begin{tabular}{|c|c|c|c|c|c|}
\hline Response & $\begin{array}{c}\text { Scenario } \\
1 \\
0.092 \mathrm{pCi} / \mathrm{g}\end{array}$ & $\begin{array}{c}\text { Scenario } \\
1 \\
.169 \mathrm{pCi} / \mathrm{g}\end{array}$ & $\begin{array}{c}\text { Scenario } \\
2 \\
.0409 \mathrm{pCi} / \mathrm{g}\end{array}$ & $\begin{array}{c}\text { Scenario } \\
3 \\
.14 \mathrm{pCi} / \mathrm{g}\end{array}$ & \\
\hline $\mathrm{H}=$ & $0.00 \mathrm{E}+00$ & $0.00 \mathrm{E}+00$ & $3.25 \mathrm{E}-04$ & 1.12E-03 & mrem/yr \\
\hline $\mathrm{Rf}=$ & $0.00 \mathrm{E}+00$ & $0.00 \mathrm{E}+00$ & $1.08 \mathrm{E}-10$ & 3.76E-10 & probability cancer death/yr \\
\hline
\end{tabular}

Exposure Scenarios \#2 and \#3 =

Inhalation Dose and Risk from Am-241 Contaminated Soil (Am-241 Concentration in Soil)

Cancer Risk Coefficients (Probability/Becquerel of Intake)

(Federal Guidance Report No. 13 [Eckerman et al.,1998])

\begin{tabular}{|l|c|c|}
\hline Mode of Intake & $\begin{array}{c}\text { Mortality } \\
(\mathbf{1 / B q})^{\mathbf{a}}\end{array}$ & $\begin{array}{c}\text { Morbidity } \\
\mathbf{( 1 / B q )}^{\mathbf{a}}\end{array}$ \\
\hline Inhalation & $9.04 \mathrm{E}-07$ & $9.58 \mathrm{E}-07$ \\
\hline Water Ingestion & $2.01 \mathrm{E}-09$ & $2.81 \mathrm{E}-09$ \\
\hline Food Ingestion & $2.56 \mathrm{E}-09$ & $3.63 \mathrm{E}-09$ \\
\hline External & $1.59 \mathrm{E}-17$ & $2.36 \mathrm{E}-17$ \\
\hline
\end{tabular}

Note - (a) the units for external exposure are $(\mathrm{kg} / \mathrm{Bq}-\mathrm{s})$ 


\section{Food Ingestion Pathway}

Assumes worst case: 20 -year old male consuming 2,952 kcal/d of food at an average rate of 1,700 kg of food per kcal (Eckerman et al., 1998). Assumes that the fraction of Am-241 transferred from the soil to food is $5.39 \mathrm{E}-4$ based upon Tables D.2 - D.4 of Yu et al., (1989). The fraction of food raised on site is adjusted per Yu et al., (1989).

Calculation of the fraction of Am-241 transferred from the soil to food:

$((160 \mathrm{~kg} / \mathrm{yr}+14 \mathrm{~kg} / \mathrm{yr}$ fruits, vegetables, grain, leafy vegetables [Table D.2]) $\times 1.0 \mathrm{E}-3 \mathrm{Am}-241$ transfer rate $($ Table D.3) $)+((63 \mathrm{~kg} / \mathrm{yr}$ of meat [Table D.2] $\times 5 \mathrm{E}-5 \mathrm{Am}-241$ transfer rate for beef (Table D.4) $)+((92 \mathrm{~kg} / \mathrm{yr}$ of milk [Table D.2] x 2E-6 Am-241 transfer rate for milk (Table D.4))/(174+63+92) $=\quad 0.000539009=\mathrm{Bv}$

The dose and risk from ingestion of food contaminated with Am-241 is calculated as follows:

$\mathrm{H}=\mathrm{C} \times \mathrm{Bv} \times \operatorname{Ir} \times \mathrm{T} \times \mathrm{DCF} \times 3,700 \times 0.25 \times \mathrm{Fu}$

$\mathrm{R}=\mathrm{C} \times \mathrm{Bv} \times \operatorname{Ir} \times \mathrm{T} \times \mathrm{Rf} \times \mathrm{Fu}$ where

$\mathrm{C}=\mathrm{Am}-241$ concentration in soil $(\mathrm{pCi} / \mathrm{g})$

$\mathrm{Bv}=$ fraction of Am-241 transferred from soil to food (Yu et. al., 1989) = 5.39E-04 pCi/g (vegetation)/pCi/g (soil))

$\mathrm{Ir}=$ food ingestion rate $=(2,952 \mathrm{kcal} / \mathrm{d}) /(1,700 \mathrm{kcal} / \mathrm{kg}$ of food $) \times 1,000 \mathrm{~g} / \mathrm{kg}=$ $1736 \mathrm{~g}$ of food/d

$\mathrm{T}=$ time dose receptor is ingesting food grown on CAU 266 leachfield = 365 days/year

DCF $=$ ingestion dose conversion factor for Am-241 (ICRP, 1995) = 2.00E-07 Sv/Bq

$3700=$ multiplication conversion factor $(\mathrm{mrem} / \mathrm{pCi}) /(\mathrm{Sv} / \mathrm{Bq})$

$0.25=$ fraction of all food grown on site $=(50 \mathrm{~m} \times 100 \mathrm{~m}) / 20,00 \mathrm{~m} 2=.25$ (Yu et al., 1989)

$\mathrm{Rf}=$ ingestion risk factor for $\mathrm{Am}-241=(2.56 \mathrm{E}-9 / \mathrm{Bq}) /(27.027 \mathrm{pCi} / \mathrm{Bq})=$ $9.47201 \mathrm{E}-11(1 / \mathrm{pCi})$

$\mathrm{Fu}=$ fraction of Am-241 taken up by roots, assumed to be fraction of Am-241 in root zone, of $0.9 \mathrm{~m}$,

Am-241 is $<9$ m BGS, Fu $=0$, otherwise 1 (Yu et al., 1989)

Food Ingestion Dose and Risk from Am-241 Contaminated Soil (Am-241 Concentration in Soil)

\begin{tabular}{|l|c|c|c|c|l|}
\hline \multicolumn{1}{|c|}{ Response } & $\begin{array}{c}\text { Scenario 1 } \\
\mathbf{0 . 0 9 2} \mathbf{~ p i / g}\end{array}$ & $\begin{array}{c}\text { Scenario 1 } \\
\mathbf{. 1 6 9} \mathbf{~ p C i / g}\end{array}$ & $\begin{array}{c}\text { Scenario 2 } \\
\mathbf{. 0 4 0 9} \mathbf{~ p C i / g}\end{array}$ & $\begin{array}{c}\text { Scenario 3 } \\
\mathbf{. 1 4} \mathbf{~ p C i} / \mathbf{g}\end{array}$ & \\
\hline $\mathrm{H}=$ & $0.00 \mathrm{E}+00$ & $0.00 \mathrm{E}+00$ & $2.58 \mathrm{E}-03$ & $8.95 \mathrm{E}-03$ & $\mathrm{mrem} / \mathrm{yr}$ \\
\hline $\mathrm{Rf}=$ & $0.00 \mathrm{E}+00$ & $0.00 \mathrm{E}+00$ & $3.31 \mathrm{E}-10$ & $1.15 \mathrm{E}-09$ & cancer death/yr \\
\hline
\end{tabular}

\section{Water Ingestion Dose and Risk from Am-241 Contaminated Soil}

This exposure scenario was not chosen due to the large distance to groundwater, very low rate of precipitation, and high evapotranspiration. 


\section{Soil Ingestion Dose and Risk from Am-241 Contaminated Soil}

$$
\begin{aligned}
& \mathrm{H}=\mathrm{C} \times \mathrm{IR} \times \mathrm{T} \times \mathrm{DR} \times \mathrm{FD} \times 3700 \\
& \mathrm{R}=\mathrm{C} \times \mathrm{IR} \times \mathrm{T} \times \mathrm{Rf} \times \mathrm{FD}
\end{aligned}
$$

where

$\mathrm{H}=$ The 50-year dose from ingesting Am-241 contaminated soil during the year of the maximum intake (mrem/year)

$R=$ The cancer mortality risk from ingestion of Am-241

$\mathrm{C}=$ is the concentration of $\mathrm{Am}-241$ in the soil, $(\mathrm{pCi} / \mathrm{g})$

IR = ingestion rate of adults from Section 6.A.1, AlHC (1994), g/day = $\quad 0.01$

$\mathrm{T}=$ the exposure time in days per year $=$

$\mathrm{DR}=$ the dose conversion factor for Am-241 Class M, Sv/Bq, ICRP Publication \#68 =

FD = Depth factor $=0$ when the cover depth, $1.07 \mathrm{~m}$, is $>0.15 \mathrm{~m}$, Equation B.5, (Yu et. al., 1989) $=\quad 0$

$\mathrm{FD}=$ Depth factor $=1$ when the cover depth is $<0.15 \mathrm{~m}$, Equation B.5, (Yu et. al., 1989) $=\quad 1$

$\mathrm{Rf}=$ the cancer risk from ingestion of $\mathrm{Am}-24,1 / \mathrm{Bq} \times \mathrm{Bq} / 27.027,(\mathrm{FGR} \# 13)=\quad 9.46 \mathrm{E}-11$

\begin{tabular}{|c|c|c|c|c|c|}
\hline \multicolumn{6}{|c|}{$\begin{array}{l}\text { Ingestion Dose and Risk from Am-241 Contaminated Soil } \\
\text { (Am-241 Concentration in Soil) }\end{array}$} \\
\hline Response & $\begin{array}{l}\text { Scenario } 1 \\
0.092 \mathrm{pCi} / \mathrm{g}\end{array}$ & $\begin{array}{l}\text { Scenario } 1 \\
0.169 \mathrm{pCi} / \mathrm{g}\end{array}$ & $\begin{array}{l}\text { Scenario } 2 \\
.0409 \mathrm{pCi} / \mathrm{g}\end{array}$ & $\begin{array}{l}\text { Scenario } 3 \\
.14 \mathrm{pCi} / \mathrm{g}\end{array}$ & \\
\hline $\mathrm{H}=$ & $0.00 \mathrm{E}+00$ & $0.00 \mathrm{E}+00$ & $1.10 \mathrm{E}-04$ & 3.82E-04 & $\mathrm{mrem} / \mathrm{yr}$ \\
\hline $\mathrm{Rf}=$ & $0.00 \mathrm{E}+00$ & $0.00 \mathrm{E}+00$ & $1.41 \mathrm{E}-11$ & $4.89 \mathrm{E}-11$ & cancer death/yr \\
\hline
\end{tabular}

$3700=$ multiplication conversion factor $(\mathrm{mrem} / \mathrm{pCi}) /(\mathrm{Sv} / \mathrm{Bq})$

\section{External Exposure Pathway}

$\mathrm{H}$ from external exposure to Am-241 = 2.34E-19 (Sv/Bq)/(s/m3) FGR \#12, (1993). Converting to familiar units: $(105 \mathrm{mrem} / \mathrm{Sv}) \times(\mathrm{Bq} / 27.027 \mathrm{pCi}) \times(3600 \mathrm{~s} / \mathrm{hr}) \times(24 \mathrm{hrs} / \mathrm{d}) \times(365.25 \mathrm{~d} / \mathrm{yr}) \times(106 \mathrm{~cm} 3 / \mathrm{m} 3) \times(1.5 \mathrm{~g} / \mathrm{cm} 3)$

$$
\begin{array}{ll}
\mathrm{H}=2.34 \mathrm{E}-19(\mathrm{~Sv} / \mathrm{Bq}) /(\mathrm{s} / \mathrm{m} 3) \times 1.751 \times 1017[(\mathrm{mrem} / \mathrm{yr}) /(\mathrm{pCi} / \mathrm{g})] /[(\mathrm{Sv} / \mathrm{Bq}) /(\mathrm{s} / \mathrm{m} 3)]=0.041(\mathrm{mrem} / \mathrm{yr}) /(\mathrm{pCi} / \mathrm{g}) \\
\mathrm{H}= & 0.04098(\mathrm{mrem} / \mathrm{yr}) /(\mathrm{pCi} / \mathrm{g}) \text { when Am-241 is at the surface } \\
\mathrm{H}= & 1.11841 \mathrm{E}-17(\mathrm{mrem} / \mathrm{yr}) /(\mathrm{pCi} / \mathrm{g}) \text { when } \mathrm{Am}-241 \text { is at } \$ 1.067 \mathrm{~m} \text { below the surface }
\end{array}
$$

Risk Coefficient for cancer mortality risk $=$ risk coefficient for cancer morbidity risk $=1.59 \mathrm{E}-17 \mathrm{~kg} /(\mathrm{Bq}-\mathrm{s})$

Converting to familiar units: $(103 \mathrm{~g} / \mathrm{kg}) \times(3.156 \times 107 \mathrm{~s} / \mathrm{yr}) \times(\mathrm{Bq} / 27.027 \mathrm{pCi})=1.168 \times 109(\mathrm{~g}-\mathrm{s}-\mathrm{Bq} / \mathrm{kg}-\mathrm{yr}-\mathrm{pCi})$

Cancer Risk Coefficient $(\mathrm{Scn} .2,3)=1.59 \mathrm{E}-17 \mathrm{~kg} / \mathrm{Bq}-\mathrm{s}) \times 1.168 \mathrm{E}+9=\quad 1.857 \mathrm{E}-08(\mathrm{risk} / \mathrm{yr}) /(\mathrm{pCi} / \mathrm{g})$

Cancer Risk Coefficient (Exposure Scenario \#1) = $\quad 5.06804 \mathrm{E}-24($ risk/yr) $/(\mathrm{pCi} / \mathrm{g})$

\section{External Dose and Risk from Am-241 Contaminated Soil} (Am-241 Concentration in Soil)

\begin{tabular}{|l|c|c|c|c|l|}
\hline Response & $\begin{array}{l}\text { Scenario 1 } \\
\mathbf{0 . 0 9 2} \mathbf{~ p C i / g}\end{array}$ & $\begin{array}{c}\text { Scenario 1 } \\
\mathbf{0 . 1 6 9} \mathbf{~ p C i / g}\end{array}$ & $\begin{array}{c}\text { Scenario 2 } \\
\mathbf{0 4 0 9} \mathbf{~ p C i / g}\end{array}$ & $\begin{array}{c}\text { Scenario 3 } \\
\mathbf{1 4} \mathbf{~ p C i} / \mathbf{g}\end{array}$ & \\
\hline $\mathrm{H}=$ & $1.03 \mathrm{E}-18$ & $1.89 \mathrm{E}-18$ & $1.68 \mathrm{E}-03$ & $5.80 \mathrm{E}-03$ & $\mathrm{mrem} / \mathrm{yr}$ \\
\hline $\mathrm{Rf}=$ & $4.66 \mathrm{E}-25$ & $8.56 \mathrm{E}-25$ & $7.60 \mathrm{E}-10$ & $2.63 \mathrm{E}-09$ & cancer death/yr \\
\hline
\end{tabular}


Total Dose From all Exposure Pathways

\begin{tabular}{|l|c|c|c|c|l|}
\hline \multicolumn{7}{|c|}{ Total Dose and Risk from Am-241 Contaminated Soil (mrem/yr) } \\
(Am-241 Concentration in Soil) \\
\hline \multirow{2}{*}{ Response } & Scenario 1 & Scenario 1 & Scenario 2 & Scenario 3 & \\
& $\mathbf{0 . 0 9 2} \mathbf{~ C C i} / \mathbf{g}$ & $\mathbf{0 . 1 6 9} \mathbf{~ P C i} / \mathbf{g}$ & $\mathbf{0 4 0 9} \mathbf{~ p C i / g}$ & $\mathbf{. 1 4} \mathbf{~ p i} / \mathbf{g}$ & \\
\hline $\mathrm{H}=$ & $1.03 \mathrm{E}-18$ & $1.89 \mathrm{E}-18$ & $4.70 \mathrm{E}-03$ & $1.63 \mathrm{E}-02$ & $\mathrm{mrem} / \mathrm{yr}$ \\
\hline $\mathrm{Rf}=$ & $4.66 \mathrm{E}-25$ & $8.56 \mathrm{E}-25$ & $1.21 \mathrm{E}-09$ & $4.20 \mathrm{E}-09$ & cancer death/yr \\
\hline
\end{tabular}

\section{CONCLUSION}

The estimated maximum dose to an individual living on the CAU 266 leachfield soil under the present conditions would be about $2.9 \mathrm{E}-18 \mathrm{mrem} / \mathrm{year}$. If the CAU 266 soil was mixed with surface soil than the dose would increase to $4.7 \mathrm{E}-3 \mathrm{mrem} / \mathrm{year}$. If all of the Am-241 was brought to the surface the dose would be about $1.63 \mathrm{E}-2 \mathrm{mrem} / \mathrm{year}$. All of these doses are less than 0.02 percent of the $100 \mathrm{mrem} / \mathrm{year}$ limit established in DOE Order 5400.5 (DOE, 1993), and less than 0.07 percent of the $25 \mathrm{mrem} /$ year established in 10 CFR 20 (NRC, 1997) for license termination.

Under all of the exposure scenarios the risk does not exceed the criterion established by the U.S. Environmental Protection Agency of $1.0 \mathrm{E}-6$ for consideration of performing remediation at a Superfund site. 


\section{References}

AIHC, see American Industrial Health Council.

American Industrial Health Council. 1994. Exposure Factors Sourcebook. Washington, DC: American Industrial Health Council.

DOE, see U.S. Department of Energy.

DOE/NV, see U.S. Department of Energy, Nevada Operations Office.

Eckerman, K.F., and J.C. Ryman. 1993. External Exposure to Radionuclides in Air, Water, and Soil, Federal Guidance Report No. 12. Oak Ridge, TN: Oak Ridge National Laboratory.

Eckerman, K.F., R.W. Leggett, C.B. Nelson, J.S. Puskin, and A.C. Richardson. 1998. Health Risks from Low-Level Environmental Exposure to Radionuclides, Federal Guidance Report No. 13, Part 1- Interim Version. Oak Ridge, TN: Oak Ridge National Laboratory.

EPA, see U.S. Environmental Protection Agency.

ICRP, see International Commission on Radiological Protection.

International Commission on Radiological Protection. 1995. Dose Coefficients for Intakes of Radionuclides by Workers, ICRP Publication No. 68. Oxford, UK: Pergamon Press.

Layton, D.W. 1993. "Metabolically Consistent Breathing Rates for Use in Dose Assessments." In Health Physics, 64 (1):23-36. Baltimore, MD: Williams and Wilkins.

McArthur, R.D., and F.L. Miller. 1989. Off-site Radiation Exposure Review Project Phase II Soils Program, Publication \#45064. Las Vegas, NV: Desert Research Institute.

NRC, see U.S. Nuclear Regulatory Commission.

Shinn, J.J. 1994. Mass Loading Data from TTR. Memorandum from J. J. Shinn, Lawrence Livermore National Laboratory, to R. Smiecinski, DOE Nevada Operations Office. Livermore, CA.

Shleien, B., L.A. Slaback Jr., B.K. Birky (Ed). 1998. Handbook of Health Physics and Radiological Health. Baltimore, MD: Williams and Wilkins.

U.S. Department of Energy. 1993. Radiation Protection of the Public and the Environment, DOE Order 5400.5. Washington, DC. 
U.S. Department of Energy, Nevada Operations Office. 1999. Corrective Action Investigation Plan for Corrective Action Unit 266: Area 25 Building 3124 Leachfield, Nevada Test Site, Nevada, DOE/NV--529-REV-1. Las Vegas, NV.

U.S. Environmental Protection Agency. 1997. Title 40 Code of Federal Regulations, Part 300, "Protection of the Environment." Washington, DC.

U.S. Nuclear Regulatory Commission. 1997. Title 10 Code of Federal Regulations, Part 20, "Standards for Protection Against Radiation." Washington, DC.

Yu, C., A.J. Zielen, J.-J. Cheng, Y.C. Yuan, L.G. Jones, D.J. LePoire, Y.Y. Wang, C.O. Loureiro, E. Gnanapragasam, E. Faillace, A. Wallo III, W.A. Williams, and H. Peterson. 1989. Manual for Implementing Residual Radioactive Material Guidelines Using RESRAD, Version 5.0. Argonne, IL: Argonne National Laboratory. 


\section{Appendix B}

\section{Radiological Walkover Survey Report}




\author{
Area 25 Building 3124 Leachfield
}

\title{
1.0 Objective
}

The objective of this radiological survey was to provide locations of surface contamination and show radiological trends to focus characterization and clean up efforts. Radiological instrumentation was used to define the nature and extent of potential radiological contaminants.

\subsection{Instruments}

- Eberline ESP-2 ${ }^{\mathrm{TM}}$ Ratemeter (SN. 1729) with 3-inch by 3-inch Sodium lodine (Nal) scintillation gamma detector (SN. 062293A)

- NE Technology model ElectraTM (SN. 1523) with model DP6BD alpha/beta probe (SN. 1401)

- Trimble Pathfinder Pro XRS ${ }^{\mathrm{TM}}$ Global Positioning System (GPS) Receiver with TSC $1^{\text {TM }}{ }^{\text {datalogger (SN. 220134393) }}$

\subsection{Data Acquisition}

Radiological background locations were selected based on soil characteristics similar to the survey area. Care was taken to avoid areas affected by known former sewer outfalls, contaminated areas, and high gamma exposure rate background areas. Background measurements were taken southeast and northwest of the CAU investigation area and are shown in Figure 1. A total of 20 background soil measurements were collected. The background soil measurement results are shown in Table 1. Radiological and GPS measurements of the background locations were performed in the same manner as the site survey described below.

A radiological survey was performed January 12 and 13, 1999, at CAS 25-05-09, leachfield. An investigation grid of 15 -meters $(\mathrm{m})$ by $15-\mathrm{m}$ was established around the leachfield with 2-m by $2-m$ internal grid spacing. Soil locations around the leachfield were chosen where the alpha/beta probe could be placed directly on the soil. The grid was marked with wooden stakes to provide uniform measurement spacing. Each measurement location was surveyed using a Trimble Pro XRS ${ }^{\top M}$ GPS. Alpha and beta measurements were collected by placing the detector directly on the surface of the soil. Gamma measurements were collected by suspending the detector $0.3 \mathrm{~m}$ above the surface of the surrounding soils. Each radiological measurement was integrated for 30 seconds and recorded on the datalogger and stored with its related GPS measurement in a combined GPS/RAD file. A total of 6 alpha, 6 beta, and 29 gamma measurements were recorded. The radiological 
measurements and Universal Transverse Mercator (North American Datum 1927) coordinates for each measured point are shown in Table 2 and 3.

\subsection{Data Processing}

The GPS/RAD data from the datalogger was downloaded to a laptop computer and the GPS measurements were post-processed using Trimble's Pathfinder Office ${ }^{\mathrm{TM}}$ software. Each GPS measurement was positionally corrected using collected realtime satellite differential signals. After post-processing, the GPS/RAD data was exported as an ASCII file and converted to an Excel spreadsheet file. The GPS/RAD data was processed using the commercial software package SURFER ${ }^{\mathrm{TM}}$ for graphical presentation. A post map was generated for alpha, beta and gamma measurements. Figure 2 shows the location and readings of the alpha, beta, and gamma measurements.

\subsection{Quality Control}

Radiological detection equipment used in this survey was checked daily as described in SQP-ITLV-460, "Daily Source and Background Check". To ensure positional accuracy, the GPS system was programmed according to the operational manual to achieve submeter accuracy. The GPS system was checked against surveyed monuments near the survey location. In addition, each positional measurement recorded was an average of at least 30 readings, which increased the positional accuracy to less than 50 centimeters. A sampling of the data was checked against source information.

\subsection{Data Review}

Radiological measurements of the surrounding soils at CAU 266, CAS 25-05-09, Area 25, Building 3124 Leachfield, shows that the diversion box has elevated alpha and beta contamination. Several alpha measurements of the soil are slightly elevated over background measurements taken on similar media in the surrounding area. Areas that show the elevated measurements are the bottom of the diversion box and the soil area around the diversion box. Factors affecting these radiological measurements included gamma shine from the nearby Test Cell, changing ambient radon levels, and instrument variations. An alpha measurement of 18 times mean background was identified at this Corrective Action Site. 


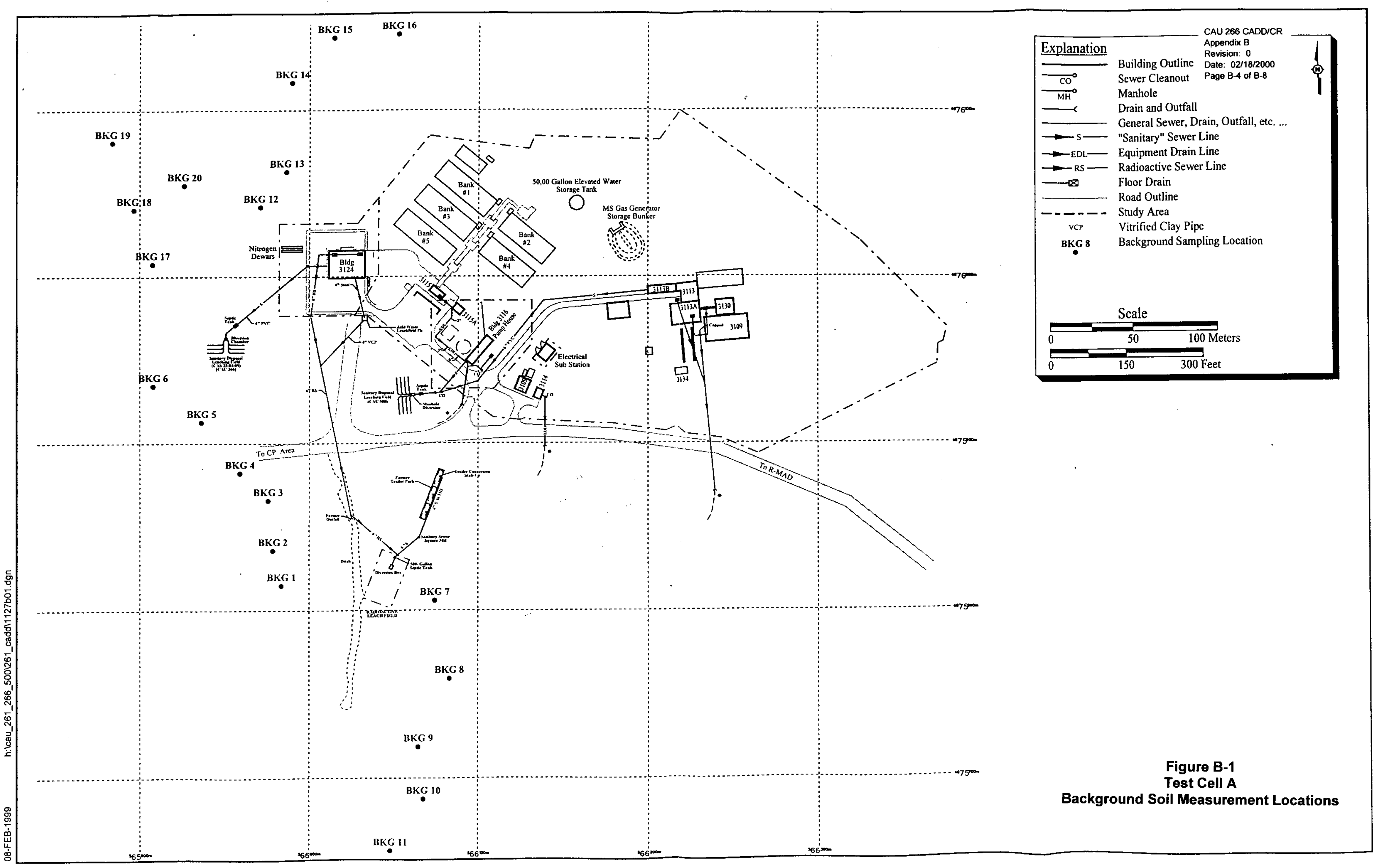


TABLE 1

Background Measurements for CAU 261, 266 and 500

\begin{tabular}{||c|c|c|c|c|c|}
\hline \multicolumn{2}{|c|}{ UTM Coordinates } & Sample & \multicolumn{3}{|c|}{ Gross Radiological Measurements } \\
\hline East & North & Number & Alpha (dpm) & Beta (dpm) & Gamma (cpm) \\
\hline 565983.31 & 4075814.86 & 1 & 49 & 1933 & 61000 \\
\hline 565978.34 & 4075835.82 & 2 & 49 & 1950 & 60600 \\
\hline 565975.54 & 4075865.73 & 3 & 60 & 1780 & 62400 \\
\hline 565958.92 & 4075882.20 & 4 & 65 & 1846 & 60900 \\
\hline 565936.30 & 4075912.72 & 5 & 54 & 1944 & 64000 \\
\hline 565907.62 & 4075934.38 & 6 & 44 & 1981 & 63500 \\
\hline 566074.38 & 4075805.71 & 7 & 0 & 2124 & 65000 \\
\hline 566083.03 & 4075759.15 & 8 & 55 & 1902 & 64600 \\
\hline 566064.78 & 4075718.54 & 9 & 38 & 1983 & 63300 \\
\hline 566067.78 & 4075687.40 & 10 & 60 & 1940 & 62000 \\
\hline 566048.18 & 4075656.46 & 11 & 49 & 1956 & 63200 \\
\hline 565970.99 & 4076041.79 & 12 & 38 & 1900 & 62700 \\
\hline 565986.29 & 4076062.87 & 13 & 33 & 1766 & 61700 \\
\hline 565989.49 & 4076116.78 & 14 & 44 & 1882 & 62200 \\
\hline 566014.42 & 4076144.15 & 15 & 65 & 1817 & 62500 \\
\hline 566052.33 & 4076146.08 & 16 & 87 & 1952 & 62200 \\
\hline 565907.39 & 4076007.61 & 17 & 49 & 1842 & 62200 \\
\hline 565896.48 & 4076040.20 & 18 & 54 & 1815 & 61200 \\
\hline 565883.96 & 4076080.55 & 19 & 9 & 1940 & 60600 \\
\hline 565926.06 & 4076054.91 & 20 & 98 & 1851 & 62000 \\
\hline Avg & & & $\mathbf{5 0}$ & $\mathbf{1 9 0 5}$ & $\mathbf{6 2 3 9 0}$ \\
\hline S.D & & & $\mathbf{2 2}$ & $\mathbf{8 4}$ & $\mathbf{1 2 5 6}$ \\
\hline 2 S.D & & & $\mathbf{4 4}$ & $\mathbf{1 6 7}$ & $\mathbf{2 5 1 2}$ \\
\hline Avg+2 S.D & & & $\mathbf{9 4}$ & $\mathbf{2 0 7 3}$ & $\mathbf{6 4 9 0 2}$ \\
\hline
\end{tabular}


TABLE 2

\section{Alpha and Beta Measurements for CAU 266}

\begin{tabular}{|c|c|c|c|}
\hline \multicolumn{2}{|c|}{ UTM Coordinates } & \multicolumn{2}{|c|}{ Gross Radiological Measurements } \\
\hline \hline East $(\mathrm{m})$ & North $(\mathrm{m})$ & Alpha (dpm) & Beta (dpm) \\
\hline \hline 565947 & 4075962 & 147 & 1855 \\
\hline 565943 & 4075956 & 70.7 & 1815 \\
\hline 565948 & 4075953 & 92.4 & 1983 \\
\hline 565954 & 4075951 & 43.5 & 1824 \\
\hline 565953 & 4075956 & 120 & 1855 \\
\hline 565948 & 4075960 & 909 & 2020 \\
\hline
\end{tabular}


TABLE 3

Gamma Measurements for CAU 266

\begin{tabular}{|c|c|c|}
\hline \multicolumn{2}{|c|}{ UTM Coordinates } & \multirow{2}{*}{$\begin{array}{c}\text { Gross } \\
\text { Gamma (cpm) }\end{array}$} \\
\hline East $(\mathrm{m})$ & North $(\mathrm{m})$ & 60100 \\
\hline 565957.2 & 4075966 & 61200 \\
\hline 565957.4 & 4075963 & 60100 \\
\hline 565956.8 & 4075960 & 59200 \\
\hline 565956.8 & 4075956 & 59500 \\
\hline 565956.4 & 4075953 & 60300 \\
\hline 565955.7 & 4075950 & 60700 \\
\hline 565952.8 & 4075950 & 61800 \\
\hline 565952.8 & 4075953 & 60800 \\
\hline 565952.3 & 4075957 & 61200 \\
\hline 565952.1 & 4075960 & 60500 \\
\hline 565952.9 & 4075962 & 59400 \\
\hline 565954.5 & 4075965 & 61000 \\
\hline 565951 & 4075965 & 58200 \\
\hline 565950.8 & 4075962 & 61100 \\
\hline 565949.9 & 4075959 & 61900 \\
\hline 565948.8 & 4075956 & 60600 \\
\hline 565948.9 & 4075953 & 61100 \\
\hline 565947.7 & 4075950 & 60500 \\
\hline 565944.4 & 4075950 & 60200 \\
\hline 565944.1 & 4075954 & 60500 \\
\hline 565944.1 & 4075956 & 60400 \\
\hline 565944.5 & 4075959 & 60500 \\
\hline 565944.8 & 4075962 & 60200 \\
\hline 565945.1 & 4075966 & 59400 \\
\hline 565941.9 & 4075966 & 60100 \\
\hline 565940.9 & 4075963 & 60200 \\
\hline 565940.7 & 4075960 & 60900 \\
\hline 565940.8 & 4075956 & 60000 \\
\hline 565940.8 & 4075954 & \\
\hline & & \\
\hline & & \\
\hline$n n$
\end{tabular}


Figure 2

CAU 266, CAS 25-05-09

Area 25 Building 3124 Leachfield

Gamma Count Rate (cpm)

Alpha and Beta Measurements (dpm/100 sq. cm)

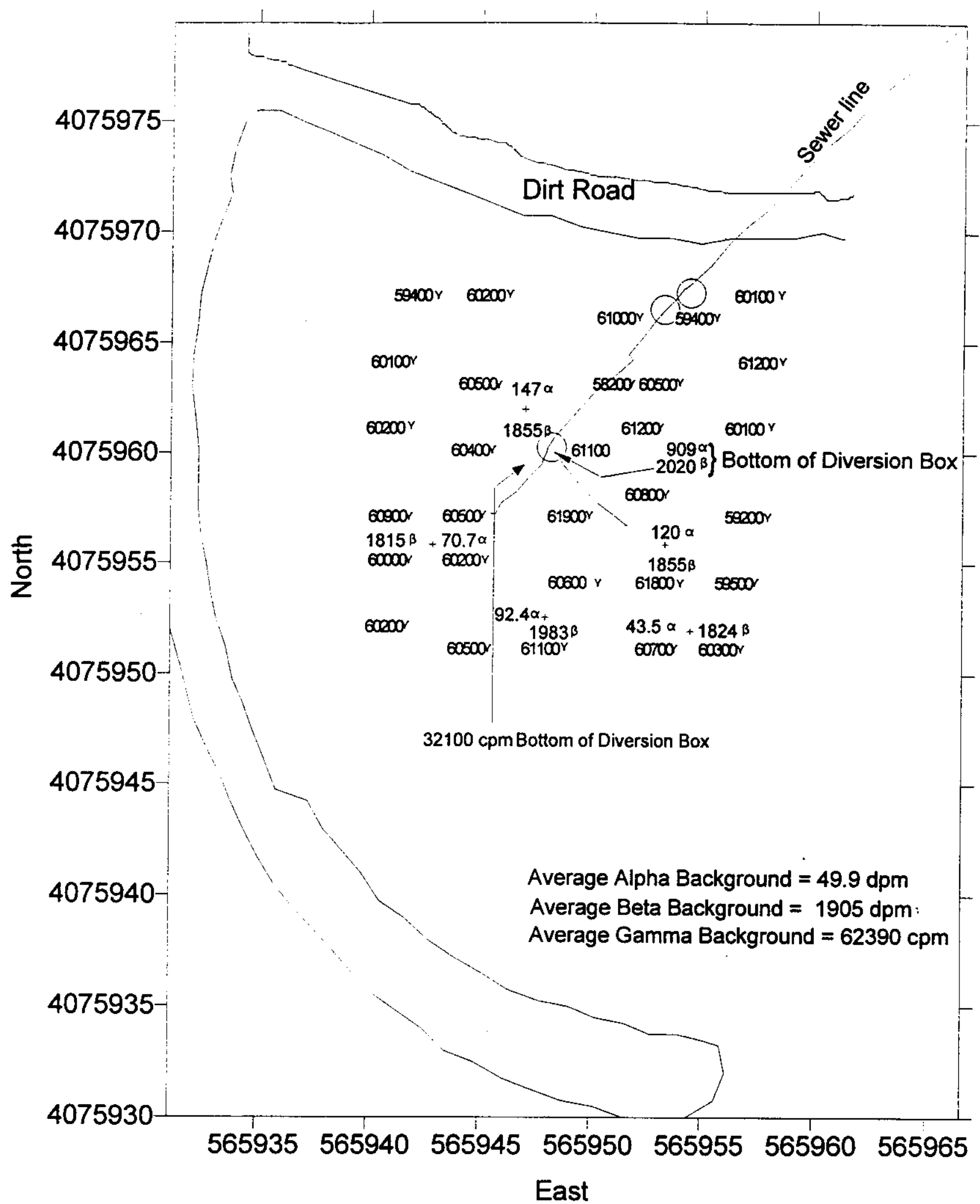




\section{Appendix C}

\section{Closure Activities Summary}




\section{CORRECTIVE ACTION UNITS (CAUs) 266 AND 500, AREA 25 TEST CELL A SEPTIC SYSTEM CLOSURES, NEVADA TEST SITE}

CAU 266 is a septic system connected to Building 3124 at the Test Cell A Complex. This system was used by the Equipment Testing Laboratory from 1962 to 1972 . It was used again from 1992 to 1995 by the Treatability Test Facility. The septic tank capacity at this site is approximately 1,000 gallons.

CAU 500 is a septic system which served Building 3116 , Building $3113 \mathrm{~B}$, as well as trailers east of the septic system outside the Test Cell A fence. The septic system was in operation from 1958 to 1966 and has an approximate capacity of 500 gallons.

The Nevada Division of Environmental Protection (NDEP) required corrective action at both CAU 266 and CAU 500 since the septic tanks contained low-level radiological and petroleum hydrocarbon waste. The requirements for closing the sites were based on the characterization data provided in the Draft Corrective Action Decision Document/Closure Report (CADD/CR) for CAU 266: Area 25 Building 3124 Leachfield. Nevada Test Site, Nevada, September 1999 and Draft CADD/CR for CAU 500: Area 25 Test Cell A Septic System, Nevada Test Site, Nevada, September 1999. The NDEP requested that the field closure activities be completed prior to submitting the Final CADD/CR for each of the sites.

Site closure was completed by conducting the following activities:

- Preparation of field documents (Site Specific Health and Safety Plan [SSHASP], Unit Work Instruction [UWI], and Field Management Plan).

- Obtain a National Environmental Policy Act (NEPA) determination and update utility clearances.

- Remove septic tank contents.

- Pressure wash tank and distribution box interiors.

- Solidify the tank contents and associated rinsate from the tank and distribution box.

- Collect verification samples from the tank rinsate.

- Excavate to expose the tanks' influent and effluent lines and grout the exposed lines.

- Grout the tanks and distribution boxes in place.

- Conduct a demarcation survey to remove radiological controls.

- Prepare a waste profile for Radioactive Waste Acceptance Program (RWAP) approval.

- Preparation of a summary letter report. 
Waste transportation and disposal is also part of the closure requirements but will be completed at a later date pending RWAP approval.

\section{PREFIELD ACTIVITIES}

Several plans and permits were prepared prior to beginning the closure activities at CAUs 266 and 500. These plans included the SSHASP with an associated Hazard Analysis and Radiological Work Permit, UWI, and a Field Management Plan. In addition, a NEPA Checklist was prepared and a site survey was performed by a biologist. Site preparation involved updating a recent utility survey, mobilization of equipment to the site, and designation of the exclusion zone and waste management area.

\section{SEPTIC SYSTEM WASTE REMOVAL}

Mobilization and site staging occurred on December 13, 1999. The septic tank contents were removed from CAUs 266 and 500 on December 14 and 15, 1999. The tanks were pressure washed concurrent with the removal of the tank contents. The associated distribution boxes were also pressure washed although little or no waste was observed in the boxes at the time of cleaning. The closure of the distribution boxes are further addressed in the "Deviation from Closure Requirements" section of this letter report.

The tank contents and associated rinse water from each of the tanks and distribution boxes were removed using a closed vacuum system. The vacuum system pumped the waste directly into 55 gallon drums. The presence of a sludge layer in CAU 500 required additional pressure washing and use of an agitator rod to break up the sludge for removal.

Rinse water samples were collected from each tank on December 15, 1999 and analyzed for total petroleum hydrocarbons as gasoline and diesel using Environmental Protection Agency (EPA) Method 8015, Modified, and gross alpha and beta spectroscopy. The samples were collected using a clean Nalgene ${ }^{\mathrm{TM}}$ sample scoop and placed into laboratory sample bottles.

The established closure criteria for the tanks was to meet the Nevada Administrative Code Action Level of 100 parts per million (ppm) for the petroleum hydrocarbons and the EPA Drinking Water Standards of 15 picoCuries per liter $(\mathrm{pCi} / \mathrm{L})$ for gross alpha and $50 \mathrm{pCi} / \mathrm{L}$ for gross beta. The results of the rinse water samples collected on December 15, 1999 did not meet the established closure standards. The tanks were pressure washed again on January 4, 2000 and rinse water samples were collected and analyzed for the same parameters. The results of the second sampling event met the closure criteria for both tanks. 
The Department of Energy (DOE) and NDEP concurred that the closure criteria for the tanks was met based on the sample results. Authorization to proceed with grout placement to close the septic systems was provided by the DOE on January 18,2000 . The rinse water results of the samples collected on January 4, 2000 are summarized in Table 1 and the data is provided in Attachment A.

TABLE 1 - SUMMARY OF SEPTIC TANK RINSE WATER RESULTS ${ }^{A}$

\begin{tabular}{|c|c|c|c|c|}
\hline $\begin{array}{c}\text { SAMPLE } \\
\text { IDENTIFICATION }\end{array}$ & $\begin{array}{l}\text { GROSS } \\
\text { ALPHA } \\
(\mathrm{PCi} / \mathrm{L}) \\
\end{array}$ & $\begin{array}{c}\text { GROSS } \\
\text { BETA } \\
(\mathrm{pCi} / \mathbf{L})\end{array}$ & $\begin{array}{c}\mathrm{TPH}^{\mathrm{B}} \\
\text { GAS } \\
(\mathrm{mg} / \mathrm{L}) \\
\end{array}$ & $\begin{array}{c}\text { TPH } \\
\text { DIESEL } \\
(\mathrm{mg} / \mathrm{L})\end{array}$ \\
\hline Closure Standard ${ }^{C}$ & $15 \mathrm{pCi} / \mathrm{L}$ & $50 \mathrm{pCiL}$ & $100 \mathrm{ppm}$ & $100 \mathrm{ppm}$ \\
\hline CAU266-002 & 4.61 & 11.1 & $<0.25$ & $<0.5$ \\
\hline CAU500-002 & 9.39 & 14.4 & $<0.25$ & 0.67 \\
\hline
\end{tabular}

A - Results represent rinse water samples from the CAU 266 septic tank (CAU266-002) and the CAU 500 septic tank (CAU500-002). Samples were collected on January 4, 2000.

$\mathrm{B}-\mathrm{TPH}=$ Total Petroleum Hydrocarbons analyzed using EPA Method 8015, Modified. Analyzed as gasoline and diesel range ( $\mathrm{Cl} 0$ to $\mathrm{C28}$ ) and reported in milligrams per liter $(\mathrm{mg} / \mathrm{L})$.

C - Closure Standard = EPA Drinking Water Standards used for Gross Alpha/Beta evaluation (40CFR 141). Nevada Administrative Code used for TPH evaluation (NAC 445A.227).

\section{SEPTIC SYSTEM CLOSURE}

Closure of the septic system was accomplished by first isolating each tank from their associated influent and effluent clay lines. The effluent clay lines from the distribution boxes from each tank were also cut. This was completed on January 19, 2000 by excavating into the lines using a backhoe. The lines were broken at a distance of approximately two to three feet from each tank and distribution box and were separated by a minimum width of two feet.

On January 20,2000, a grout/slurry mix was used to seal the ends of the influent and effluent lines. The CAU 266 and 500 tanks and distribution boxes were also filled with grout to the top of each structure.

\section{WASTE MANAGEMENT}

A total of approximately 650 gallons of waste was generated from both tank sites. The containerized waste was transported to Fluid Tech, Inc. (Fluid Tech), located at the E-MAD Facility in Area 25 on December 16, 1999 and on January 4, 2000. Fluid Tech solidified the 

Acceptance Criteria specifications.

The waste profile has been submitted for RWAP review. Contingent on approval, the waste is expected to be disposed of by February 29, 2000. Manifests and disposal documentation will be provided subsequent to disposal.

\section{DEVIATION FROM CLOSURE REQUIREMENTS}

After grouting the distribution boxes, it was realized that rinse water samples were not collected to verify that the closure criteria had been met. Greg Raab of the NDEP was informed of the deviation from the sample requirement and a teleconference was held on January 26, 2000. In the teleconference, the NDEP provided guidance to summarize the scope deviation and field activities accomplished. The NDEP also indicated that, based on the information provided in the teleconference, that the closure work completed at the sites would be acceptable. It is believed that the information provided in this letter meets the NDEP request.

Although rinse samples were not collected to verify that the closure criteria was met, it is . believed that the distribution boxes were sufficiently cleaned and do not pose a health or environmental risk based on the following:

- Pressure washing of the distribution box interior was done.

- The volume of waste that remained in each distribution box prior to pressure washing was very minimal. This was also noted to be the case during the original characterization sampling.

- The concentrations of radioisotopes and petroleum hydrocarbons detected during the original characterization analysis were very low. The characterization data is available in the Draft CADD/CR reports completed for CAU 266 and CAU 500.

- The assumption that since the work completed was able to meet the closure criteria for the tanks that the same procedure would have met the closure criteria for the distribution boxes. It is important to note that the original concentrations of the tank contents were much higher than the original concentrations of the distribution box residual.

- The boxes are structurally intact indicating that nothing has been or will be released into the underlying soil.

- The boxes are sealed to the surface with grout.

\section{SUMMARY}

Closure was completed at CAUs 266 and 500 by removing the low level radioactive waste and petroleum hydrocarbon waste from the tanks and distribution boxes. Verification samples of the 
final rinse water from the septic tanks were collected and met the established closuge C-6 of C-33 Rinse water samples from the associated distribution bos criteria.

All lines leading to the tanks and distribution boxes were cut and the ends sealed with grout. The tanks and associated distribution boxes were also filled with grout. It is anticipated, based on the work completed, that the site can be closed without further corrective action requirements. 
CAU $266 \mathrm{CADD} / \mathrm{CR}$

Appendix $\mathrm{C}$

Revision: 0

Date: $02 / 18 / 2000$

Page C-7 of $\mathrm{C}-33$

\section{ATTACHMENT A}

\section{RINSE WATER ANALYTICAL DATA}




\section{Bechtel Nevada}

To: W. F. Johnson

From: $\quad$ L. W. Hatcher Analytical Services Laboratory, 295-7109
Date: January 20, 2000

No.: $\quad 2150-A L-00-0196$

\section{$\begin{array}{ll}\text { Subject: } & \text { DATA REPORT FOR SAMPLE DELIVERY GROUP (SDG) D015 } \\ \text { Project No. } 04001\end{array}$}

Analytical Services Laboratory's (ASL) data results for the gross alpha and gross beta analyses of one water sample submitted to the laboratory on January 05, 2000 are included with a copy of this memorandum to J. F. Bonn. The service statement summarizing the costs and work performed by the Analytical Services Laboratory is also included.

A Bechtel Environmental Integrated Data Management System (BEIDMS) deliverable was also requested for this sample set. The BEIDMS electronic data file was loaded on January 20, 2000 in BEIDMS under document identifier "CAU 266 RAD".

This sample was also submitted for non-radiological analyses and logged in under SDG V745. Preliminary data were faxed to the client on January 10,2000. The final data report will be submitted once the final data package has been received from the subcontract lab and reviewed.

Please direct any questions you may have to your Client Service Representative, Ted Redding, at 295-7220.

LWH:dh

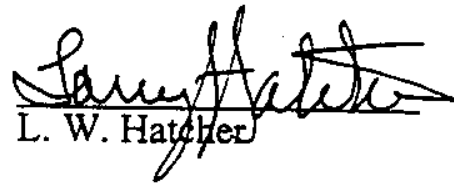

Subject Code: ENV3

cc: Correspondence Control, NLV008

J. F. Bonn, (results enc.), NTS306

D. M. Van Etten NLV082

ASL SDG D015, (results enc.) NTS273

ASL SDG V745, NTS273 
ANALYTICAL SERVICES LABORATORY

P.O.Box 3936, N. Las Vegas, NV 89036

Reported to: Environmental Restoration

P.O. Box 98521

M/S NTS306

Las Vegas
Analysis: Gross Alpha

Analysis: Gross Beta
Report Date: 10-JAN-00

Sample Delivery Group: D015

Batch: N509

Program: 720

\begin{tabular}{|c|c|c|c|c|c|c|c|c|c|c|c|c|c|c|c|c|}
\hline 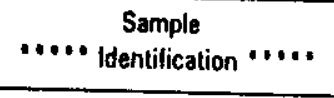 & Isotope & Analysis & Result & Erior & $\begin{array}{l}\text { Oual } \\
\text { Flag }\end{array}$ & MOC & $\begin{array}{l}\text { Result } \\
\text { Units }\end{array}$ & $\begin{array}{c}\text { Analysis } \\
\text { Dale }\end{array}$ & \begin{tabular}{|c} 
Sample \\
Coll Date
\end{tabular} & Size & \begin{tabular}{|r|} 
Size \\
Units \\
\end{tabular} & $\begin{array}{c}\text { Type } \\
\text { Matrix } \\
\end{array}$ & \begin{tabular}{|l|} 
Traces \\
Yield \%
\end{tabular} & $\begin{array}{l}\text { Spike } \\
\text { Recv \% }\end{array}$ & \begin{tabular}{|l|} 
System \\
Detector
\end{tabular} & $\begin{array}{l}\text { Packet-litem } \\
\text { Sample }\end{array}$ \\
\hline CAU266-002 & Gross Alpha & Gross A & $4.61 E+00$ & $1.6 \mathrm{E}+00$ & & $2.1 E+00$ & $\mathrm{pCi} / \mathrm{L}$ & $01-07-00$ & 01.04 .00 & $6.78 E+02$ & $\mathrm{ml}$ & Water & & & $14-01$ & $H 2298 \cdot 0.75230$ \\
\hline OA REA & Gross Alpha & Gross A & $1.64 \mathrm{E}-02$ & $2.3 \mathrm{E}-01$ & u & $4.2 \mathrm{E}-01$ & $\mathrm{pCin}$ & 01.06 .00 & $01-05.00$ & $9.00 E+02$ & $\mathrm{ml}$ & $O A, W$ & & & 14.01 & $\mathrm{H} 2300-0.08473$ \\
\hline QA SP:GROSSAB 06 & Gross Alpha & Gross A & $7.38 E+00$ & 7.3E-01 & & $4.2 \mathrm{E}-01$ & $p C i / L$ & $01-06-00$ & $01-01-99$ & $9.00 E+02$ & $\mathrm{ml}$ & $\alpha A, w$ & & 112.2 & 14.01 & H2300-1.08474 \\
\hline CAU266.002 & Gross Beta & Gross $\mathrm{B}$ & $1.11 E+01$ & $1.3 E+00$ & & $1.7 E+\infty$ & $\mathrm{pCi} / \mathrm{L}$ & 01.07 .00 & $01-04.00$ & $6.78 E+02$ & $\mathrm{ml}$ & Water & & & 14.01 & $\mathrm{H} 2298 \cdot 0 \cdot 75230$ \\
\hline OA REA & Gross Beta & Gross B & $6.76 \mathrm{E}-02$ & 6.7E-01 & $v$ & $1.1 E+\infty$ & $\mathrm{pCi} / \mathrm{L}$ & $01-06.00$ & $01-05.00$ & $9.00 E+02$ & $\mathrm{ml}$ & $\mathrm{OA}, \mathrm{W}$ & & & 14.01 & $\mathrm{H} 2300 \cdot 0-08473$ \\
\hline OA SP:GROSSAB $\quad 06$ & Gross Beta & Gross B & $5.43 E+00$ & $8.2 \mathrm{E} \cdot 01$ & & $1.1 E+\infty 0$ & $\mathrm{pCi} / \mathrm{L}$ & 01.06 .00 & 01.01 .99 & $9.00 E+02 \mid$ & $\mathrm{ml}$ & $0 A, W$ & & 91.5 & 14.01 & $\mathrm{H} 2300 \cdot 1.08474$ \\
\hline
\end{tabular}

Comment:

Data generated from analyses of one sample submitted to the

laboratory on January 5, 2000. Preliminary data were faxed to

the client on January 10, 2000.

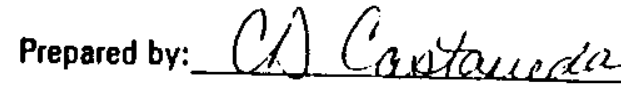
Date: $01 / 1 /(20$

Approved by: $\frac{Q}{0}=$ Attaken

\section{Qualification Flags:}

$E=$ Estimated Quantity

$H=$ High Recovery for Sample

$\mathrm{J}=$ Result is less than the RDL

$L=$ Low Recovery for Sample

$\mathrm{P}=$ Preliminary Results

$\mathrm{Q}=$ Bad Instrument Quality Control, Result is OK

$\mathrm{R}=$ Results are Unusable, Resampling is Necessary

$\mathrm{U}=$ Result is less than Minimum Detectable Activity
Note: Error is the 2.0 Sigma Error 
Job Number: 23220

Facllity ID: NTS

Sampling Event: SEPTIC TANK CLOSURES COC Number: CAU 266 RAD

Laboratory: ASL
Cooler 10:

Cooler Temp:

Number of Coolers:

Cooler Units:
Delivery Order ID:

Page C-10 of C-33

Sign

SEIR NO.: CAU 266-2-0

Charge Code: C7D424AA

Sampled By:

equested Analysis:

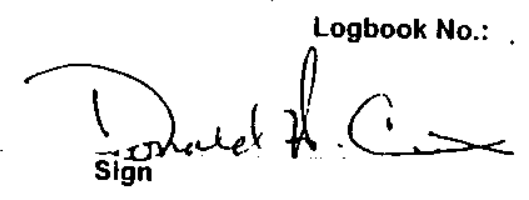

Print

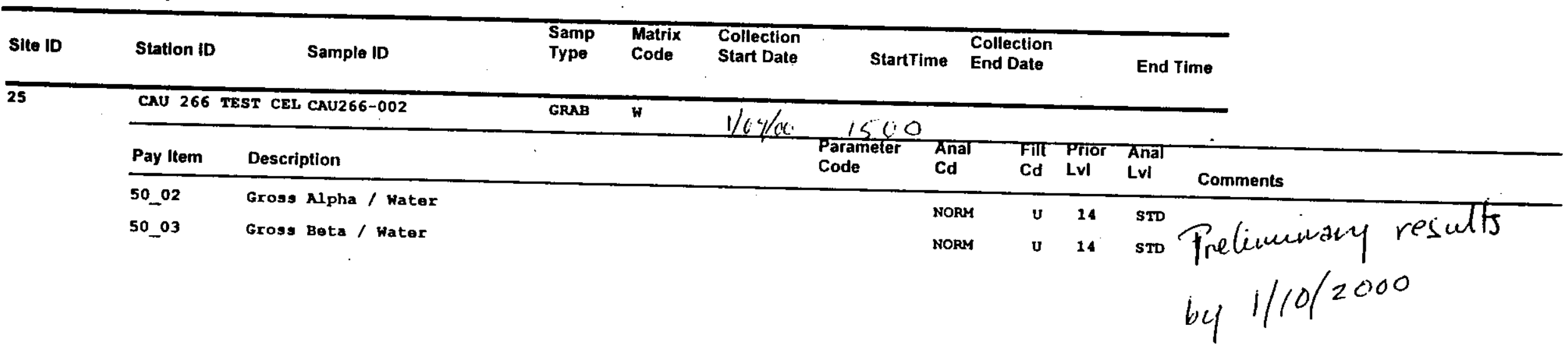


Containers Included on C.o.C.:

\begin{tabular}{|c|c|c|c|c|c|c|}
\hline Sample ID & Container 10 & Preservative & $\begin{array}{l}\text { Filler } \\
\text { Code }\end{array}$ & $\mathrm{pH}$ & Contalner Type & Comments \\
\hline CAU266-002 & CAU266-00201 & coor $4 \mathrm{C}$ & & & GLASS BOTTLE & rogs Alpha $\&$ \\
\hline
\end{tabular}




\section{Transfer Information:}

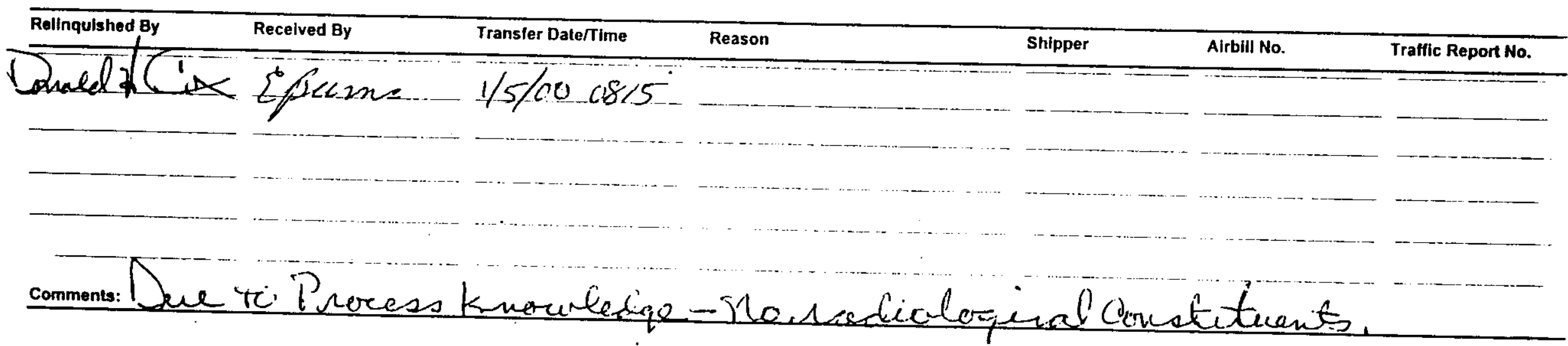

\section{Potential Contamination Yes No}

Radiological

Chemical

$-\cdots$


DATE: $1-4 / 10$ NO. A005927 Bechtel N'evada

Radiological Control Department- Ramatrol

\section{Material Clearance}

MONITOR: E jerrevine esyle ISSUING ORGANIZATION:

REQUESTING ORGANIZATION:

SURVEY LOCATON: TEST' Coll-

FROM:

TO:

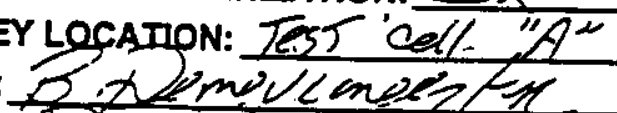

Page $\mathrm{C}-13$ of $\mathrm{C}-33$

THIS MATERIAL HAS EEEN SURVEYED FOR RADIOACTIVIT AND MEETS DOE STANDARDS FOR RELEASE TO PUELIC USE

\section{DESCRIPTION:}

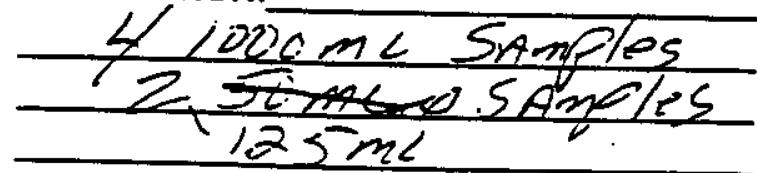

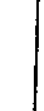

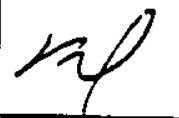

REMARKS:

THIS TAG EXPIRES 7 DAYS FROM ISSUE DATE 
CLIENT:

Bechtel Nevada

P.O. Box 98521 , M/S NTS273

ATTN:

Las Vegas, NV 89193-8521

Ted Redding

PROJECT NAME: $\quad$ V745

PROJECT NUMBER: 17777

NEL ORDER ID: L0001027

Attached are the analytical results for samples in support of the above referenced project.

Samples submitted for this project were not sampled by NEL Laboratories. Samples were received by NEL in good condition, under chain of custody on $1 / 5 / 00$.

Should you have any questions or comments, please feel free to contact our Client Services department at (702) 657-1010.
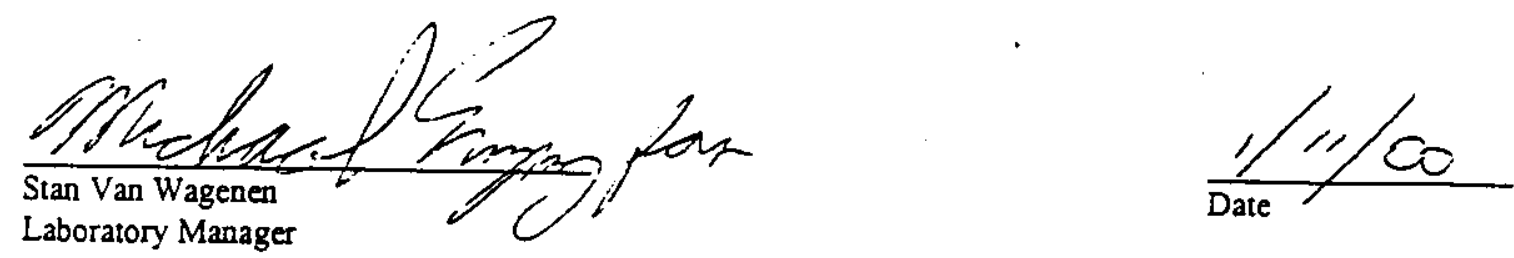

\section{CERTIFICATIONS:}

\begin{tabular}{|c|c|c|c|c|c|c|c|}
\hline & Reno & Las Vegas & S. California & & Reno & Vegas & S. Califomia \\
\hline Arizona. & AZ0520 & AZOS 18 & $A Z 0605$ & Idaho & Certified & Centified & \\
\hline California & 1707 & 2002 & 2264 & Montana & Certified & Certified & \\
\hline $\begin{array}{l}\text { US Amy Corps } \\
\text { of Engineers }\end{array}$ & Certified & Cenified & & $\begin{array}{l}\text { Nevada } \\
\text { L.A.C.S.D. }\end{array}$ & NV033 & NV052 & $\begin{array}{l}\text { CA084 } \\
10228\end{array}$ \\
\hline
\end{tabular}


- ' ' NEL LABORATORIES

\begin{tabular}{llll}
\hline CLIENT: & Bechtel Nevada & CLIENT ID: & CAU266-002 \\
PROJECT ID: & V745 & DATE SAMPLED: $1 / 4 / 00$ \\
PROJECT \#: & 17777 & NEL SAMPLE D: L0001027-01 \\
TEST: & Purgeable Total Petroleum Hydrocarbons by Mod8015-GRO & \\
METHOD: & EPA 8015M & ANALYST: & BJV-Las Vegas Division \\
MATRIX: & Aqueous & EXTRACTED: & $1 / 6 / 00$ \\
DIIUTION: & 1 & ANALYZED: & $1 / 6 / 00$
\end{tabular}

\section{PARAMETER}

Gas Range Organics

$\frac{\text { Result }}{\mathrm{ND}}$
Reporting
Limit

QUALITY CONTROL DATA:

Surrogate

a,a,a-Trifluorotoluene

\% Recovery

115
CAU 266 CADD/CR

Appendix $\mathrm{C}$

Revision: 0

Date: $02 / 18 / 2000$

Page C-15 of C-33

ND - Not Detected

This report shall not be reproduced except in full, without the written approval of the laboratory. 


\begin{tabular}{lllll}
\hline CLIENT: & Bechtel Nevada & CLIENT ID: & Method Blank & Appendix C \\
PROJECT ID: & V745 & DATE SAMPLED: & NA & Date: 02/18/2000 \\
PROJECT \#: & 17777 & NEL SAMPLE ID: & 000106GAS-BLK & Page C-16 of C-33
\end{tabular}

TEST: Purgeable Total Petroleum Hydrocarbons by Mod8015-GRO METHOD: $\quad$ EPA 8015M

MATRIX: Aqueous

ANAIYST: $\quad$ BJV - Las Vegas Division

EXTRACTED: $\quad 1 / 6 / 00$

ANALYZED: $\quad 1 / 6 / 00$

\begin{tabular}{|c|c|c|}
\hline PARAMETER & Result & $\begin{array}{c}\text { Reporting } \\
\text { Limit } \\
\end{array}$ \\
\hline Gas Range Organics & ND & $0.25 \mathrm{mg} / \mathrm{L}$ \\
\hline \multicolumn{3}{|c|}{ QUALITY CONTROL DATA: } \\
\hline$\frac{\text { Surrogate }}{\text { a.a.a-Trifluorotoluene }}$ & $\frac{\% \text { Recovery }}{111}$ & $\frac{\text { Acceptable Range }}{70-130}$ \\
\hline
\end{tabular}

ND - Not Detected

This report shall not be reproduced except in full, without the written approval of the laboratory. 
CLIENT: Bechtel Nevada

PROJECT ID: V745

Date: 02/18/2000

PROJECT \#: $\quad 17777$

TEST: Total Extractable Petroleum Hydrocarbons by EPA Method 8015M, December 1996

METHOD: EPA $8015 \mathrm{M}$

ORDER ID: L0001027

MATRIX: Aqueous

ANALYST: SLB - Las Vegas Division

\begin{tabular}{|c|c|c|c|c|c|c|}
\hline $\begin{array}{l}\text { CLIENT } \\
\text { SAMPLE ID } \\
\end{array}$ & $\begin{array}{c}\text { SAMPLE } \\
\text { DATE }\end{array}$ & $\begin{array}{c}\text { NEL } \\
\text { SAMPLE ID } \\
\end{array}$ & $\begin{array}{c}\text { RESULT } \\
\mathrm{mg} / \mathrm{L}\end{array}$ & .R. & $\begin{array}{c}\text { Reporting } \\
\text { Limit }\end{array}$ & $\begin{array}{l}\text { Surrogate } \\
\text { Recovery } \\
\text { E }^{*}\end{array}$ \\
\hline 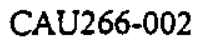 & $1 / 4 / 00$ & L0001027-0I & ND & $\bar{D}$ & $0.5 \mathrm{mg}$ & $102 \%$ \\
\hline
\end{tabular}

\section{C:R.: Carbon Range}

D Diesel Range Organics (C10 to C28).

QUALITY CONTROL DATA (Total for Diesel Range):

\section{Sample ID}

Blank. 000105TP -BLK

LCS. 000105TPHW-LCS

LCSD, 000105TPHW-LCSD

* Surrogate used was Octacosane, acceptance limits 60-131\%.
Result Acceptable Range Surrogate Recovery* Sample Number

$\begin{array}{llllll}\text { ND } & < & 0.5 \mathrm{mg} / \mathrm{L} & 80 & \% & \text { NA } \\ 74 & \% & 57 & -109 \% & 97 \% & \text { NA } \\ 74 & \% & 57 & -109 \% & 95 . \% & \text { NA }\end{array}$

ND - Not Detected

This report shall not be reproduced except in full, without the written approval of the laboratory. 
AU 266 CADD/CR

Appendix C

01/03/<๖j0

TS - PRODUCTION

Job Number: 23220

Facility ID: NTS

Sampling Event: SEPTIC TAKK CLOSURES

COC Number: CAU 266 RAD

Laboratory: NEL23

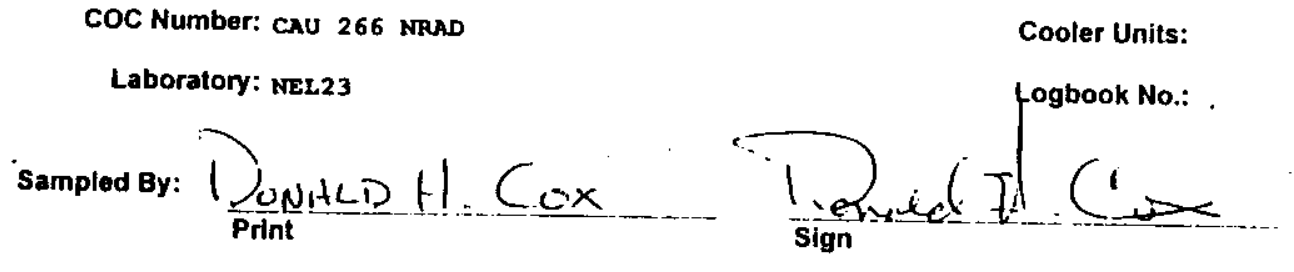

CHAIN OF CUSTODY RECORD: TAU 266 NAD LQ00102.7

Cooler ID:

Cooler Temp:

Number of Coolers:

Cooler Units:
Delivery Order ID:

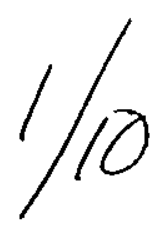

Print
Date: 02/18/2000
Page C-18 of C-33
SEIR NO.: CAU 266-2-0

Charge Code: C7D124MA

Requested Analysis:

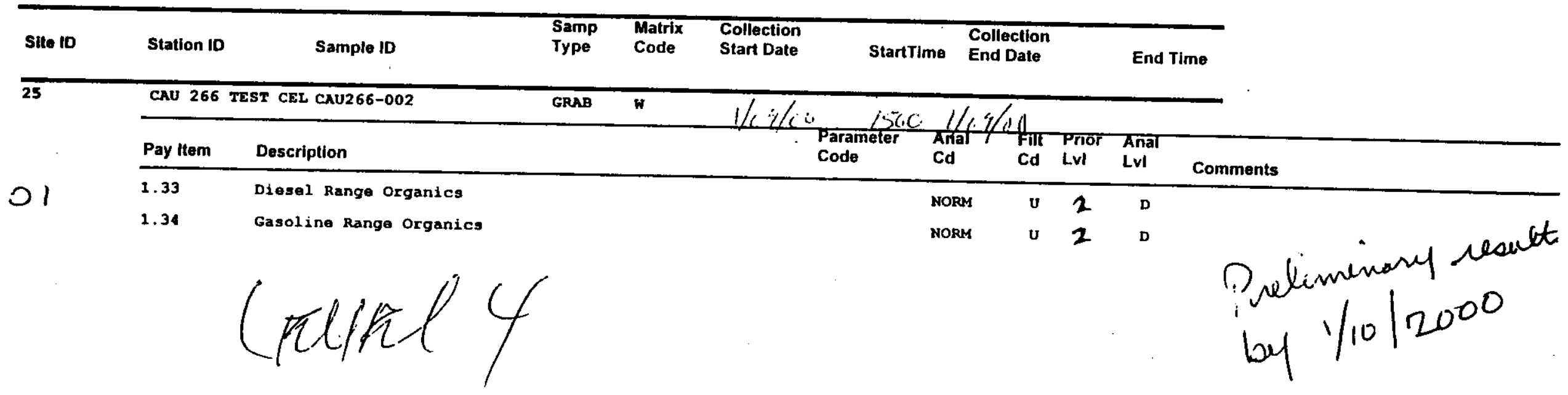


Containers Included on C.o.C.:

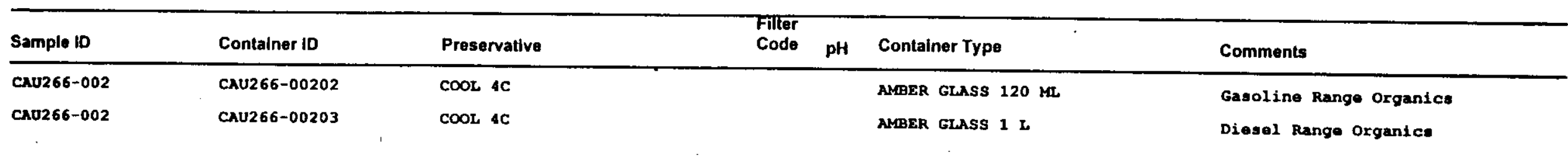


Transfer Information:

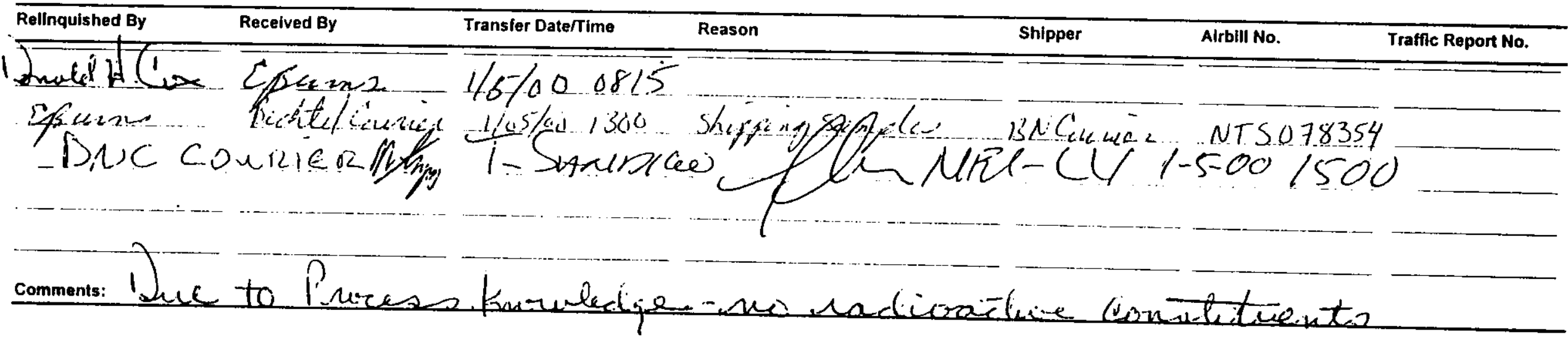

Potential Contamination Yes No

$\begin{gathered}\text { Radiological } \\ \text { Chemical }\end{gathered}-$


To: W. F. Johnson

From: L. W. Hatcher

Analytical Services Laboratory, 295-7109
Date: January 20, 2000

No.: 2150-AL-00-0197

\section{Subject: $\begin{aligned} & \text { DATA REPORT FOR SAMPLE DELIVERY GROUP (SDG) D016 } \\ & \text { Project No. } 04001\end{aligned}$}

Analytical Services Laboratory's (ASL) data results for the gross alpha and gross beta analyses of one water sample submitted to the laboratory on January 05, 2000 are included with a copy of this memorandum to J. F. Bonn. The service statement summarizing the costs and work performed by the Analytical Services Laboratory is also included.

A Bechtel Environmental Integrated Data Management System (BEIDMS) deliverable was also requested for this sample set. The BEIDMS electronic data file was loaded on January 20, 2000 in BEIDMS under document identifier "CAU 500-1 RAD".

This sample was also submitted for non-radiological analyses and logged in under SDG V746. Preliminary data were faxed to the client on January 10,2000 . The final data report will be submitted once the final data package has been received from the subcontract lab and reviewed. Please direct any questions you may have to your Client Service Representative, Ted Redding, at
$295-7220$.

LWH:dh

Subject Code: ENV3

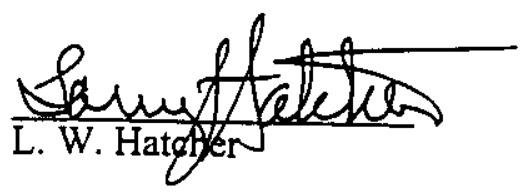

cc: Correspondence Control, NLV008

J. F. Bonn, (results enc.), NTS306

D. M. Van Etten NLV082

ASL SDG D016, (results enc.) NTS273

ASL SDG V746, NTS273 


\section{Bechtel Nevada Corporation}

ANALYTICAL SERVICES LABORATORY

P.O.Box 3936, N. Las Vegas, NV 89036

Reported to: Environmental Restoration

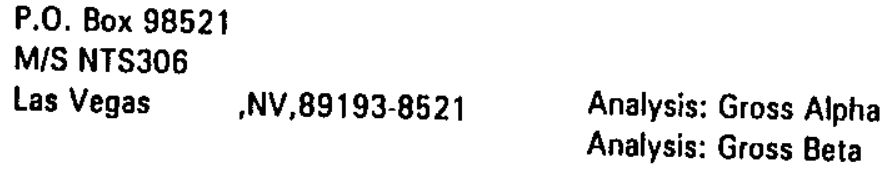

Report Date: 10-JAN-00

Sample Delivery Group: D016

Batch: N509

Report No. :

Program: 720

\begin{tabular}{|c|c|c|c|c|c|c|c|c|c|c|c|c|c|c|c|c|}
\hline ..... Identification ...... & Isotope & Analysis & Result & Error & $\begin{array}{l}\text { Qual } \\
\text { Flag }\end{array}$ & MDC & $\begin{array}{l}\text { Result } \\
\text { Units }\end{array}$ & $\begin{array}{c}\text { Analysis } \\
\text { Date }\end{array}$ & $\begin{array}{c}\text { Sample } \\
\text { Coll Date }\end{array}$ & Size & $\begin{array}{l}\text { Size } \\
\text { Unils }\end{array}$ & $\begin{array}{c}\text { Type } \\
\text { Mattix }\end{array}$ & $\begin{array}{l}\text { Tracer } \\
\text { Yield } \%\end{array}$ & $\begin{array}{l}\text { Spike } \\
\text { Recv \% }\end{array}$ & $\begin{array}{l}\text { System } \\
\text { Detector }\end{array}$ & $\begin{array}{l}\text { Packet-Item } \\
\text { Sample }\end{array}$ \\
\hline CAU500.002 & Gross Alpha & Gross A & $9.39 E+00$ & $2.3 E+\infty$ & & $2.5 E+00$ & pCi/L & $01.07-00$ & 01.04 .00 & $5.30 E+02$ & $\mathrm{ml}$ & Water & & & 14.01 & H2299-0-75232 \\
\hline QA REA & Gross Alpha & Gross A & $1.64 \mathrm{E}-02$ & $2.3 \mathrm{E}-01$ & $\mathrm{u}$ & $4.2 \mathrm{E}-01$ & $\mathrm{pCi}$ & $01-06-00$ & $01-05-00$ & $9.00 E+02$ & $\mathrm{ml}$ & $a A, W$ & & & $14-01$ & $H 2300-0.08473$ \\
\hline OA SP:GROSSAB 06 & Gross Alpha & Gross A & $7.38 E+00$ & $7.3 \mathrm{E} \cdot 01$ & & $4.2 \mathrm{E} \cdot 01$ & $\mathrm{pCin}$ & $04-06-00$ & 01.01 .99 & $9.00 E+02$ & $\mathrm{ml}$ & $0 A, W$ & & 112.2 & 14.01 & $\mathrm{H} 2300-1.08474$ \\
\hline CAU500-002 & Gross Beta & Gross $B$ & $1.44 E+01$ & $1.6 E+00$ & & $2.2 \mathrm{E}+00$ & $p \operatorname{Cin}$ & $01.07-00$ & $01.04-00$ & $5.30 E+02$ & $\mathrm{ml}$ & Watet & & & $14-01$ & H2299-0.75232 \\
\hline QA REA & Gross Beta & Gross B & $6.76 \mathrm{E}-02$ & $6.7 E-01$ & U & $1.1 E+\infty$ & pCin & 01.06 .00 & $01-05-00$ & $9.00 E+02$ & $\mathrm{ml}$ & $O A, W$ & & & 14.01 & $\mathrm{H} 2300 \cdot 0.08473$ \\
\hline OA SP:GROSSAB & Gross Beta & Gross B & $5.43 E+00$ & $8.2 \mathrm{E}-01$ & & $1.1 E+00$ & $p \mathrm{Ci} / \mathrm{L}$ & $01.06-00$ & $01-01.99$ & $9.00 E+02$ & $\mathrm{ml}$ & $a A, W$ & & 91.5 & 14.01 & $\mathrm{H} 2300 \cdot 1.08474$ \\
\hline
\end{tabular}

Comment:

Data generated from analyses of one sample submitted to the laboratory on January 5, 2000. Preliminary data were laxed to the client on January 10, 2000.

Prepared by: (M) (avtaceda Date: $0 / 1 / 100$

Approved by: $\bigcap_{0}$ thetehesen Date: $1 / 11 / 0$ o
Qualification Flags:

$E=$ Estimated Quantity

$H=$ High Recovery for Sample

$\mathrm{J}=$ Result is less than the RDL

$L=$ Low Recovery for Sample

$P=$ Preliminary Results

$\mathbf{Q}=$ Bad Instrument Quality Control, Result is OK

$R=$ Results are Unusable, Resampling is Necessary

$\mathrm{U}=$ Result is less than Minimum Detectable Activity
Note: Error is the 2.0 Sigma Error 
Job Number: 23220

Cooler ID:

Facillity ID: NTS

Sampling Event: SEPTIC TANK CLOSURES COC Number: CAU 500-1 RAD

Laboratory: AsL
Cooler Temp:

Number of Coolers:

Cooler Units:

Logbook No.:
Page C-23 of $\mathrm{C}-33$

Delivery Order ID:

SEIR NO.: CAU 500-1-0

Sampled By: T.DNALD $+1 . C O x+1$

equested Analysis:

Prin

Sign

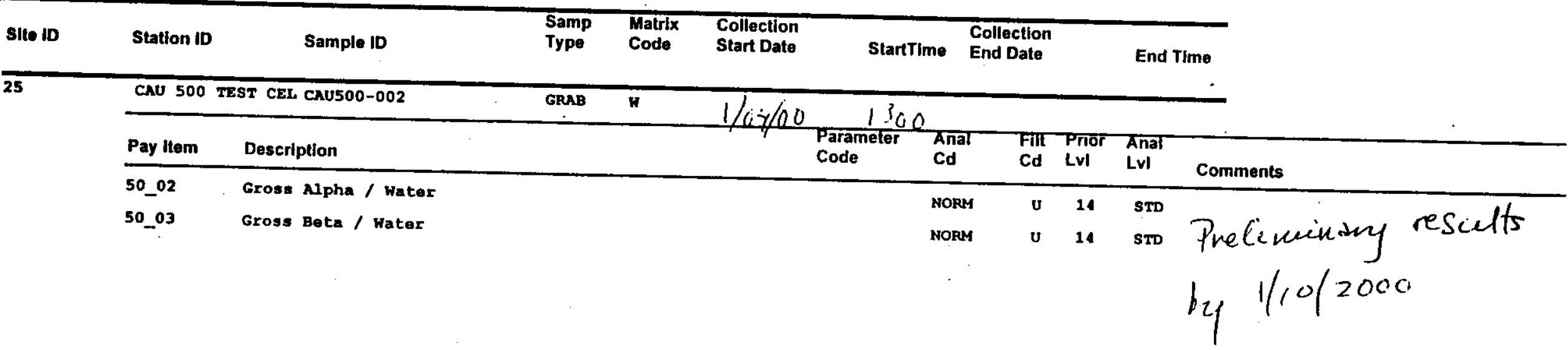


Containers Included on C.o.C.:

\begin{tabular}{|c|c|c|c|c|c|}
\hline caus00-002 & CAU500-00201 & $\operatorname{coOL} 4 \mathrm{C}$ & & GLASS BOTTLE 1 L & Gross Alpha \& Bata / Water \\
\hline
\end{tabular}


$01 / 03 / 2000$

NTS - PRODUCTION CHAIN OF CUSTODY RECORD: CAU 500-1 RAD

AU $266 \mathrm{CADD} / \mathrm{CR}$

Appendix $\mathrm{C}$

Revision: 0

Date: 02/18/2000

Transfer Information:

Page C-25 of C-33

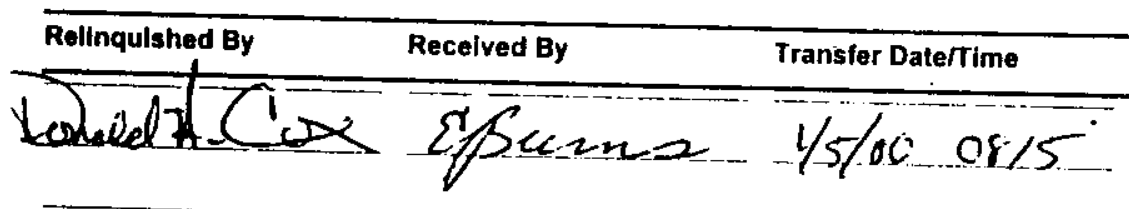

Reason

Shipper

Alrbill No.

Traffic Report No.

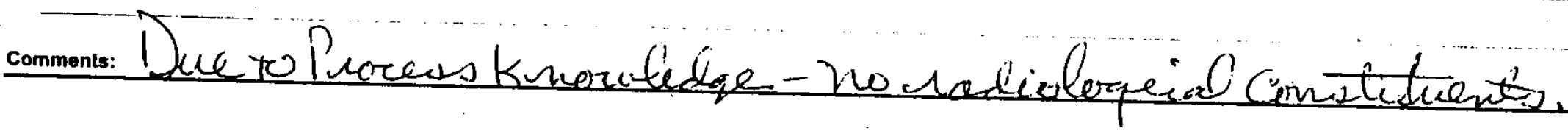

Potential Contamination Yes No

Radiological

Chemical

This package conforms to the conditions and limitations specified in 49 CFR 173.421 for excepted radioactive material, limited quantity, n.o.s., UN2910. 
DATE: $\angle-\angle H O$ NO. A005927

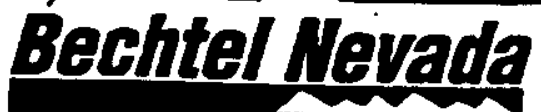

Radlological Control Departmont-Ramatrol

\section{Material Clearance}

MONITOR: 3 Jemevine evy,

ISSUING ORGANIZATION:

REQUESTING ORGANIZATION:

SURVEY LOCARON: TEST' COll.

FROM:

TO:

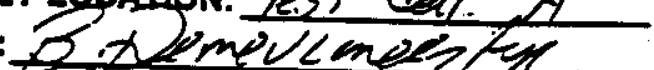

cor

THIS MATERIAL HAS BEEN SURVEYED

FOR RADIOACTMITY AND MEETS

DOE STANDARDS FOR RELEASE TO PUBLC USE

\section{DESCRIPTION:}

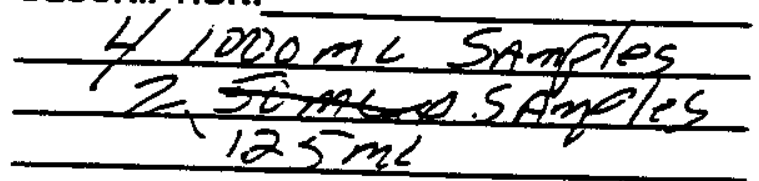

REMARKS:

THIS TAG EXPIRES 7 DAYS FROM

ISSUE DATE

CONY 1: HO RECORDS WE NTSASO

COPY 2: WTH TTEMS TUT WERE CIENRE 
$\begin{array}{ll}\text { CLIENT: } & \text { Bechtel Nevada } \\ & \text { P.O. Box 98521, M/S NTS273 } \\ & \text { Las Vegas, NV 89193-8521 } \\ \text { ATTN: } & \text { Ted Redding }\end{array}$

PROJECT NAME: $\quad$ V746 PROJECT NUMBER: 17777
NEL ORDER ID: L0001026

Attached are the analytical results for samples in support of the above referenced project.

Samples submitted for this project were not sampled by NEL Laboratories. Samples were received by NEL in good condition, under chain of custody on 1/5/00. Should you have any questions or comments, please feel free to contact our Client Services deparment at (702)
$657-1010$.

Some results have been flagged as follows:

F3 - Hydrocarbon pattem atypical of diesel.
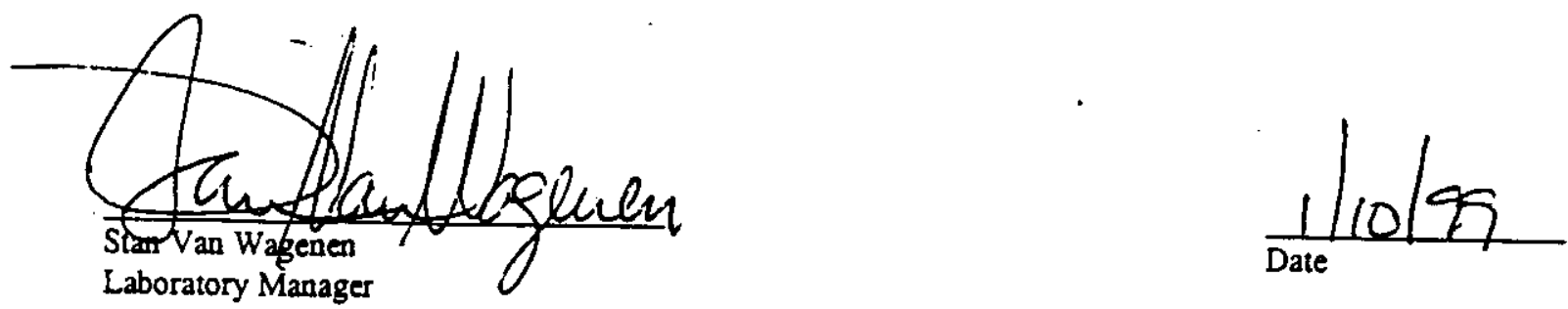

\section{CERTIFICATIONS:}

\begin{tabular}{|c|c|c|c|c|c|c|c|}
\hline $\begin{array}{l}\text { Arizona } \\
\text { California } \\
\text { US Army Corps } \\
\text { of Engineers }\end{array}$ & $\begin{array}{l}\text { Reno } \\
\text { AZ0520 } \\
1707 \\
\text { Certified }\end{array}$ & $\begin{array}{l}\text { Las Vegas } \\
\text { AZ0518 } \\
2002 \\
\text { Certified }\end{array}$ & $\begin{array}{l}\text { S. Califomia } \\
\text { AZ0605 } \\
2264\end{array}$ & $\begin{array}{l}\text { Idaho } \\
\text { Montana } \\
\text { Nevada } \\
\text { L.A.C.S.D. }\end{array}$ & $\begin{array}{l}\text { Reno } \\
\text { Centified } \\
\text { Certified } \\
\text { NV033 }\end{array}$ & $\begin{array}{l}\text { Las Vegas } \\
\text { Certified } \\
\text { Certified } \\
\text { NVOS2 }\end{array}$ & $\begin{array}{l}\text { CA084 } \\
10228\end{array}$ \\
\hline
\end{tabular}


NEL LABORATORIES

\begin{tabular}{|c|c|c|c|c|}
\hline $\begin{array}{l}\text { CLIENT: } \\
\text { PROJECT ID: } \\
\text { PROJECT \#: }\end{array}$ & $\begin{array}{l}\text { Bechtel Nevada } \\
\text { V746 } \\
17777\end{array}$ & $\begin{array}{l}\text { CLIENT ID: } \\
\text { DATE SAMPLED: } \\
\text { NEL SAMPLE ID: }\end{array}$ & $\begin{array}{l}\text { CAU500-002 } \\
\text { L/4/00 } \\
\text { L0001026-01 }\end{array}$ & $\begin{array}{l}\text { CAU } 266 \text { CADD/CR } \\
\text { Appendix C } \\
\text { Revision: } 0 \\
\text { Date: } 02 / 18 / 2000 \\
\text { Page C-28 of C-33 }\end{array}$ \\
\hline $\begin{array}{l}\text { TEST: } \\
\text { METHOD: } \\
\text { MATRIX: } \\
\text { DILUTION: }\end{array}$ & $\begin{array}{l}\text { Purgeable Total } \\
\text { EPA 8015M } \\
\text { Aqueous } \\
1\end{array}$ & $\begin{array}{l}\text { Mod8015-GRO } \\
\text { ANALYST: } \\
\text { EXTRACTED: } \\
\text { ANALYZED: }\end{array}$ & $\begin{array}{l}\text { BJV - Las Veg } \\
1 / 6 / 00 \\
1 / 6 / 00\end{array}$ & gas Division \\
\hline $\begin{array}{l}\text { PARAMETER } \\
\text { Gas Range Organi }\end{array}$ & & $\begin{array}{l}\text { Result } \\
\mathrm{ND} \\
\end{array}$ & & $\begin{array}{l}\text { Reporting } \\
\text { Limit } \\
0.25 \mathrm{mg} / \mathrm{L} \\
\end{array}$ \\
\hline \multicolumn{5}{|c|}{ QUALITY CONTROL DATA: } \\
\hline$\frac{\text { Surrogate }}{a, a, a-T r i f l u o r o t o}$ & & $\frac{\% \text { Recovery }}{116}$ & & $\frac{\text { Acceptable Ran }}{70-130}$ \\
\hline
\end{tabular}

VD - Not Detected

This report shall not be reproduced except in full, without the written approval of the laboratory. 
NEL LABORATORIES

\begin{tabular}{llll}
\hline CLIENT: & Bechtel Nevada & CLIENT ID: & Method Blank \\
PROJECT D: & V746 & DATE SAMPLED: NA \\
PROJECT \#: & 17777 & NEL SAMPLE ID: 000106 GAS-BLK
\end{tabular}

CAU 266 CADD/CR

Appendix $\mathrm{C}$

Revision: 0

Date: 02/18/2000

Page $\mathrm{C}-29$ of $\mathrm{C}-33$

TEST: $\quad$ Purgeable Total Petroleum Hydrocarbons by Mod8015-GRO

METHOD: EPA 8015M

MATRIX: Aqueous

ANALYST: $\quad$ BJV - Las Vegas Division

EXTRACTED: $\quad 1 / 6 / 00$

ANALYZED: $\quad 1 / 6 / 00$

\begin{tabular}{|c|c|c|}
\hline$\frac{\text { PARAMETER }}{\text { jas Range Organics }}$ & $\frac{\text { Result }}{\text { ND }}$ & $\begin{array}{l}\text { Reporting } \\
\frac{\text { Limit }}{0.25 \mathrm{mg} / \mathrm{L}}\end{array}$ \\
\hline QUALITY CONTROL & & \\
\hline$\frac{\text { Surrogate }}{\text { a.a.a-Trifluorotoluene }}$ & $\frac{\% \text { Recovery }}{111}$ & $\frac{\text { Acceptable Range }}{70-130}$ \\
\hline
\end{tabular}

ND - Not Detected

This report shall not be reproduced except in full, without the written approval of the laboratory. 


$\begin{array}{ll}\text { CLIENT: } & \text { Bechtel Nevada } \\ \text { PROJECT ID: } & \text { V746 } \\ \text { PROJECT \#: } & 17777\end{array}$

TEST: Total Extractable Petroleum Hydrocarbons by EPA Method 8015M, December 1996

METHOD: $\quad$ EPA 8015M

ORDER ID: $\quad$ L0001026

MATRIX: Aqueous

ANALYST: SLB - Las Vegas Division

CLIENT

\begin{tabular}{ccc}
$\begin{array}{c}\text { SAMPLE } \\
\text { DATE }\end{array}$ & NEL RESULT \\
SAMPLE ID & mg/L \\
\hline
\end{tabular}

SAMPLE ID

CAU500-002

1/4/00 L0001026-01 $\frac{0.67 \text { F3 }}{\text { D }}$

\section{Reporting Surrogate}

C.R. Limit Recovery ${ }^{*}$ EXTRACTED ANALYZED

$0.5 \mathrm{mgl} \quad 90 \% \quad 1 / 5 / 00 \quad 1 / 6 / 00$

\section{C.R.: Carbon Range}

D Diesel Range Organics (C10 to $\mathrm{C} 28$ ).

QUALITY CONTROL DATA (Total for Diesel Range):

Sample ID

Blank, 000105TP -BLK

LCS, 000105TPHW-LCS

LCSD, 000105TPHW-LCSD

* Surrogate used was Octacosane, acceptance limits 60-131\%.

\begin{tabular}{|c|c|c|c|c|c|c|}
\hline Result & & Accept & table Range & Surrogate & Recovery* & Sampie Number \\
\hline $\mathrm{ND}$ & & $<$ & $0.5 \mathrm{mg} / \mathrm{L}$ & 80 & $\%$ & NA \\
\hline $\begin{array}{l}74 \\
74\end{array}$ & $\%$ & $\begin{array}{l}57 \\
57\end{array}$ & $-109 \%$ & & $\%$ & NA \\
\hline $\begin{array}{l}74 \\
\text { eptanc }\end{array}$ & $\%$ & $\begin{array}{l}57 \\
\text { ts } 60 .\end{array}$ & $-109 \%$ & & $\%$ & $\mathrm{NA}$ \\
\hline
\end{tabular}

ND - Not Detected

This report shall not be reproduced except in full, without the written approval of the laboratory. 
$01 / 03 / 2000$
Job Number: 23220

Facility ID: NTS

COC Number: CAU 500-1 NRAD

Laboratory: NEL23

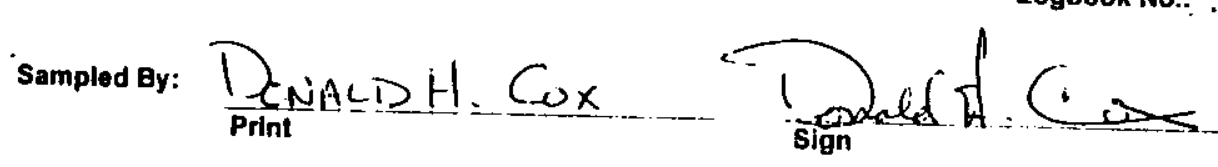

Cooler ID:

Cooler Temp:

Number of Coolers:

Cooler Units:

Logbook No.: .
NUS - PRODUCTION

Sampling Event: sePTIC TANK CLOSURES

CHAIN OF CUSTODY RECORD: CAU 500-1 NRAD LQ001026

Requested Analysis:

This package conforms to the conditions and limitations specified in 49 CR 173.421 for excepted radioactive material, limited quantity, n.0.s., UN2910. 
Contalners Included on C.o.C.:

\begin{tabular}{|c|c|c|c|c|c|}
\hline Sample ID & Contalner ID & Preservative & $\begin{array}{l}\text { Filter } \\
\text { Code pH }\end{array}$ & Container Type & Comments \\
\hline $\begin{array}{l}\text { Caus00-002 } \\
\text { Cau500-002 }\end{array}$ & $\begin{array}{l}\text { Cxu500-00202 } \\
\text { cau500-00203 }\end{array}$ & $\begin{array}{l}\text { cool ic } \\
\text { cool 4c }\end{array}$ & & $\begin{array}{l}\text { MMER GLASS } 120 \mathrm{ML} \\
\text { AMBER GLASS } 1 \text { i }\end{array}$ & $\begin{array}{l}\text { Gasoline Range Organice } \\
\text { Diesel Range Organte. }\end{array}$ \\
\hline
\end{tabular}


Transfer Information:

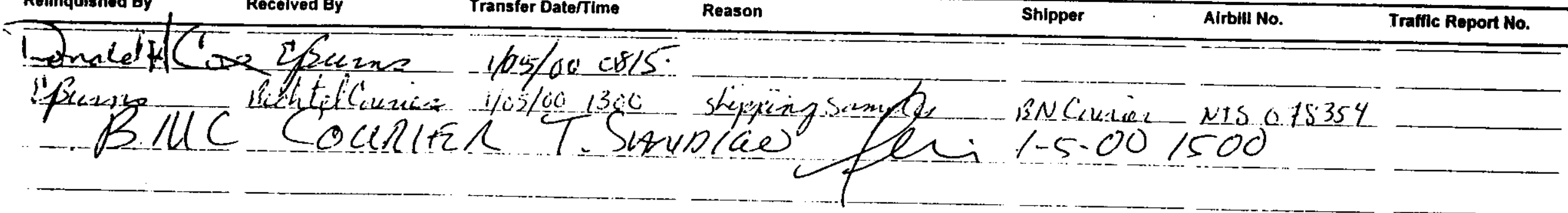

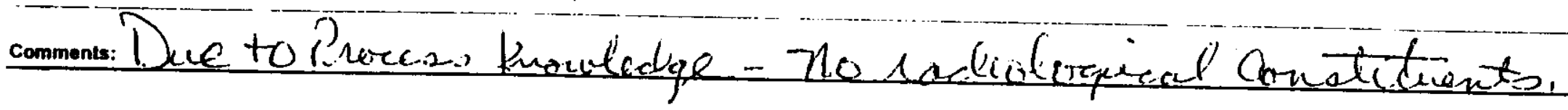

\section{Potentlal Contamination Yes No}

Radiological

Chemical

二二 


\section{Appendix D}

\section{Waste Generator Approval Process Flow Diagram}

This information was copied directly from Appendix A of the Nevada Test Site

Waste Acceptance Criteria 


\section{Waste Generator Approval Process Flow Diagram}

The following diagram identifies key process steps. Operations and logistics may influence the order in which these activities are conducted. A Site Visit (Section 2.6) may be requested by the generator at any time during the process.

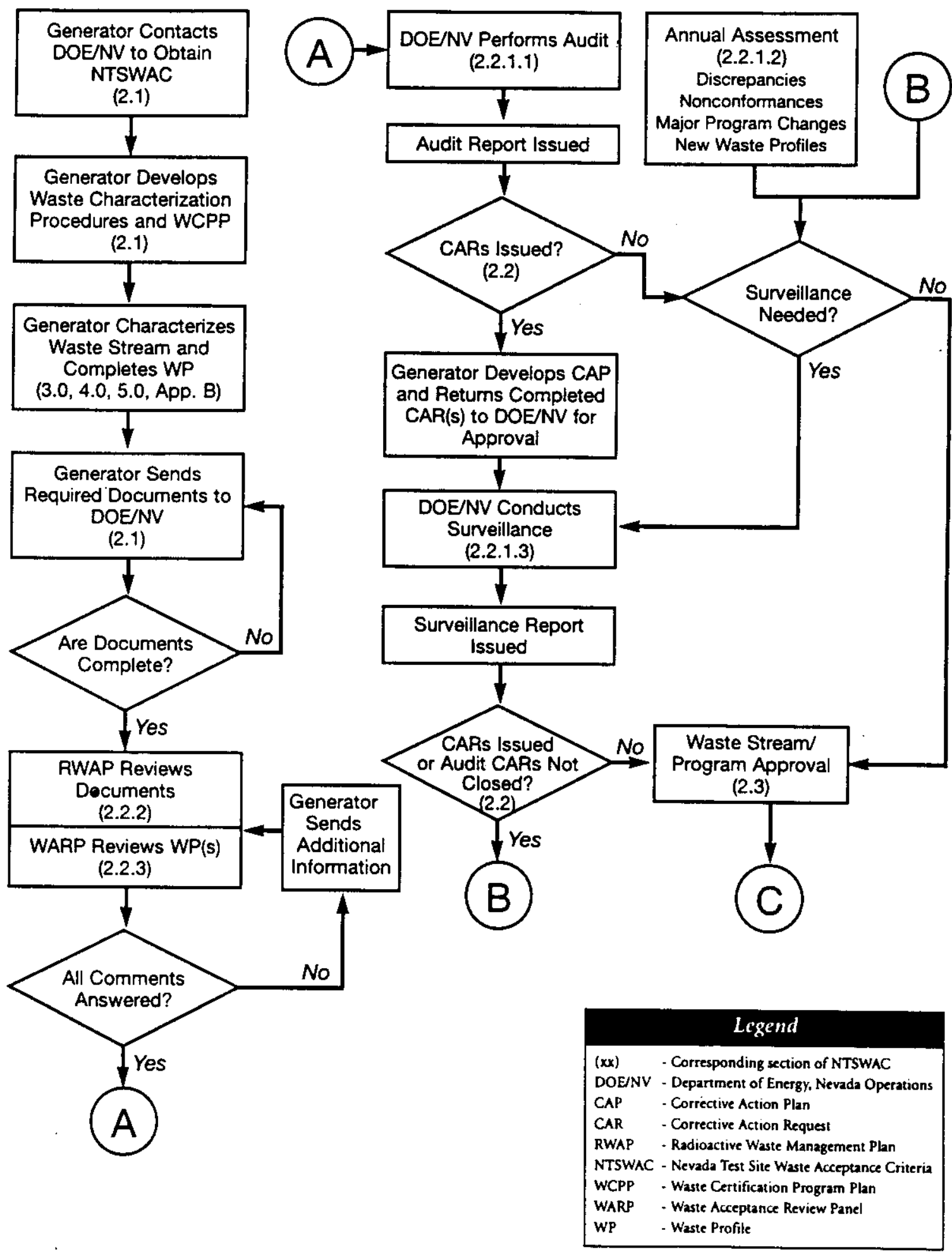



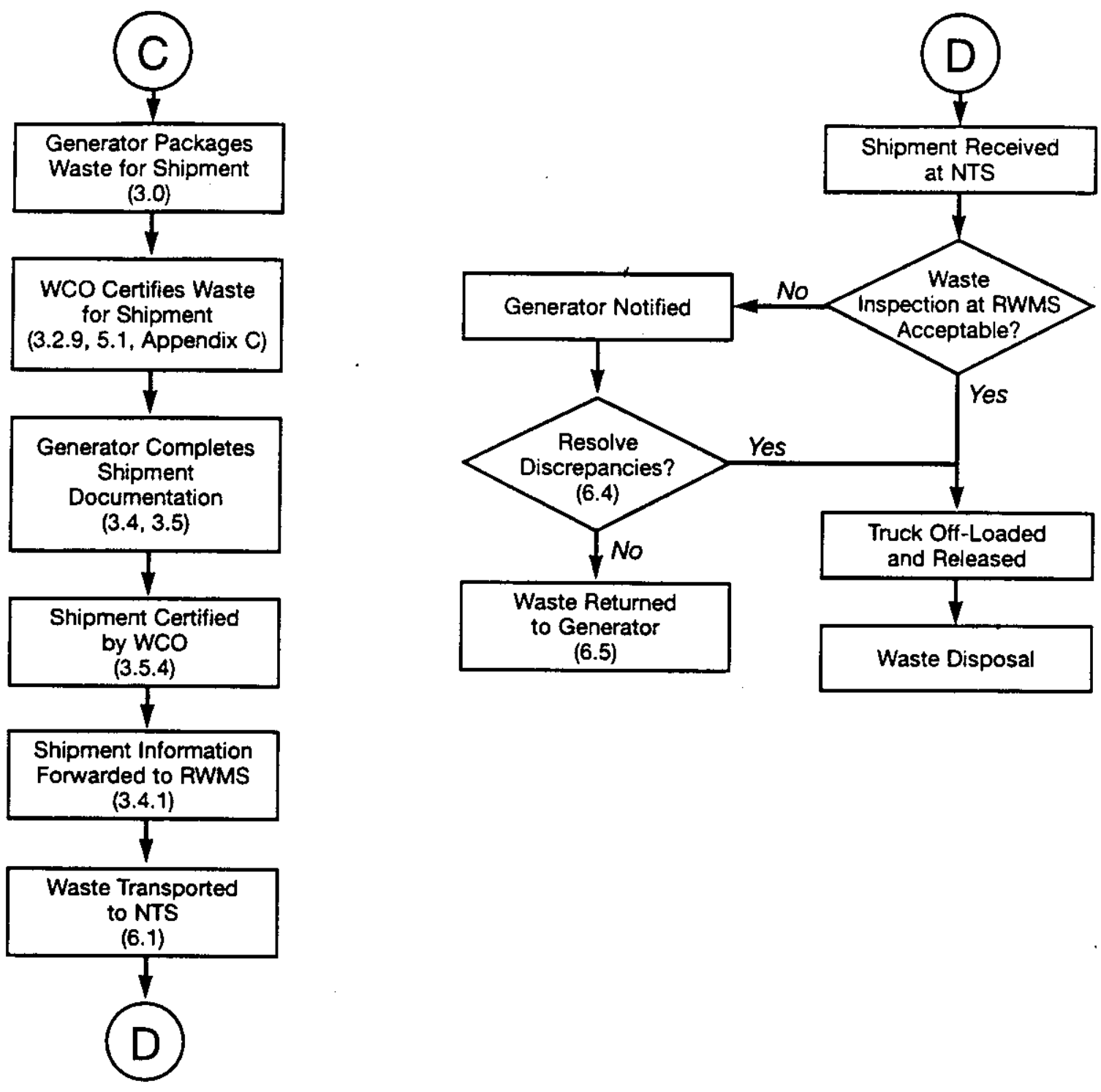

\section{Legend}

(xx) - Corresponding section of NTSWAC

DOE/NV - Department of Energy, Nevada Operations

CAP - Corrective Action Plan

CAR - Corrective Action Request

RWAP - Radioactive Waste Management Plan

NTSWAC - Nevada Test Site Waste Acceptance Criteria

WCPP - Waste Certification Program Plan

WARP - Waste Acceptance Review Panel

WP - Waste Profile 


\section{Section 2.2.2 Waste Profile}

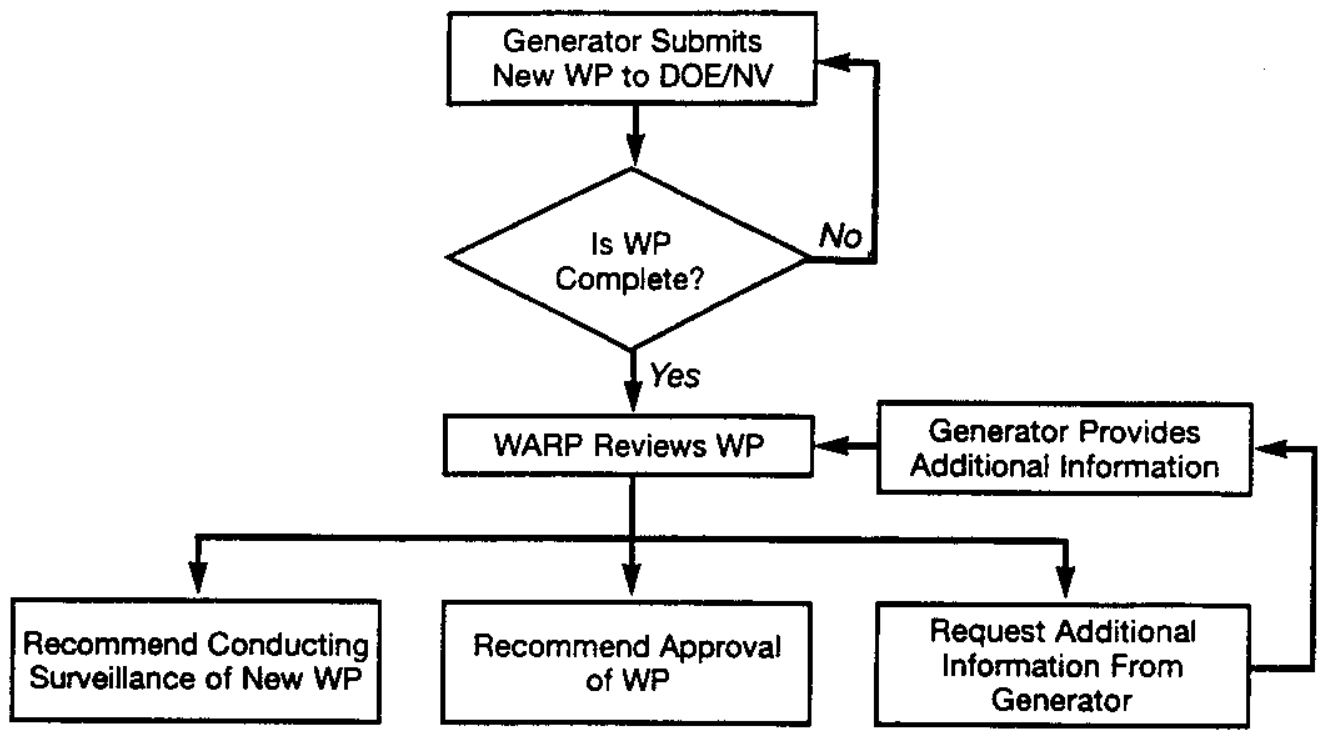

\section{Section 2.2.3 Document and Personnel Changes}

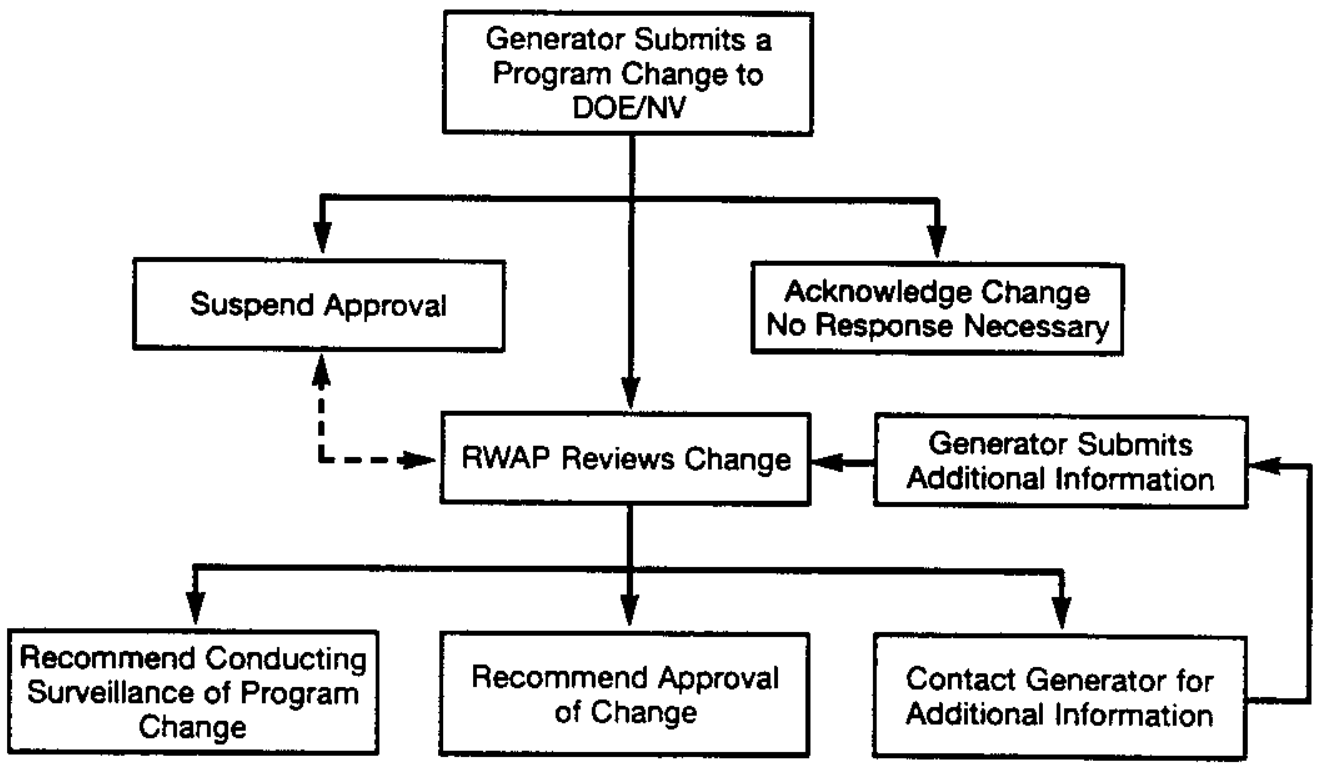

\begin{tabular}{|ll|}
\hline \multicolumn{1}{|c|}{ Legend } \\
\hline (xx) & - Corresponding section of NTSWAC \\
DOE/NV & - Department of Energy, Nevada Operations \\
CAP & - Corrective Action Plan \\
CAR & - Corrective Action Request \\
RWAP & - Radioactive Waste Management Plan \\
NTSWAC & - Nevada Test Site Waste Acceptance Criteria \\
WCPP & - Waste Certification Program Plan \\
WARP & - Waste Acceptance Review Pancl \\
WP & - Waste Profile \\
\hline
\end{tabular}


CAU 266 CADD/CR

Appendix E

Revision: 0

Date: 02/18/2000

Page E-1 of E-3

\section{Appendix E}

\section{Response to NDEP Comments}


NEVADA ENVIRONMENTAL RESTORATION PROJECT

DOCUMENT REVIEW SHEET

\begin{tabular}{|c|c|c|c|c|c|}
\hline \multicolumn{4}{|c|}{$\begin{array}{l}\text { 1. Document Title/Number: Draft Corrective Action Decision Document/Closure Report for Corrective Action } \\
\text { Unit 266: Area } 25 \text { Building } 3124 \text { Leachfield, Nevada Test Site, Nevada }\end{array}$} & \multicolumn{2}{|l|}{ 2. Document Date: September 1999} \\
\hline \multicolumn{4}{|c|}{ 3. Revision Number: 0} & \multicolumn{2}{|l|}{ 4. Originator/Organization: IT Corporation } \\
\hline \multicolumn{4}{|c|}{ 5. Responsible DOE/NV ERP Project Mgr.: Janet Appenzeller-Wing } & \multicolumn{2}{|l|}{ 6. Date Comments Due: $10-20-99$} \\
\hline \multicolumn{6}{|c|}{ 7. Review Criteria: Full } \\
\hline \multicolumn{4}{|c|}{ 8. Reviewer/Organization/Phone No.: Gregory A. Raab/NDEP (702) 486-2867 } & \multicolumn{2}{|l|}{ 9. Reviewer's Signature: } \\
\hline $\begin{array}{l}\text { 10. Comment } \\
\text { Number/ } \\
\text { Location }\end{array}$ & 11. Type* & 12. Comment & & 3. Comment Response & $\begin{array}{l}14 . \\
\text { Accept }\end{array}$ \\
\hline $\begin{array}{l}\text { 1. Page ES2, } \\
\text { 2nd Bullet }\end{array}$ & & $\begin{array}{l}\text { As the last paragraph states, the contents of the septic tank and } \\
\text { distribution box must be removed before the site can be closed. } \\
\text { Until and unless this is done, the site cannot be considered closed. }\end{array}$ & \multicolumn{2}{|c|}{$\begin{array}{l}\text { The contents of the septic tank were removed and the septic } \\
\text { tank and distribution box were pressure washed. Verification } \\
\text { samples were obtained, analyzed, and reported from the septic } \\
\text { tank and are shown in Appendix C of this CADD/CR. }\end{array}$} & Yes \\
\hline $\begin{array}{l}\text { 2. Page } 7 \text {, } \\
\text { Section } 2.3\end{array}$ & & $\begin{array}{l}\text { Need for Corrective Action: This states there must be corrective } \\
\text { action; therefore, there must be a Corrective Action Plan for closure. }\end{array}$ & \multicolumn{2}{|c|}{$\begin{array}{l}\text { No corrective action plan is necessary because closure activities } \\
\text { have been completed and reported in this CADD/CR. }\end{array}$} & Yes \\
\hline $\begin{array}{l}\text { 3. Page } 8,2 \text { nd } \\
\text { Bullet }\end{array}$ & & Same comment as \#1. & \multicolumn{2}{|c|}{$\begin{array}{l}\text { The contents of the septic tank were removed and the septic } \\
\text { tank and distribution box were pressure washed. Verification } \\
\text { samples were obtained, analyzed, and reported from the septic } \\
\text { tank and are shown in Appendix C of this CADD/CR. }\end{array}$} & Yes \\
\hline $\begin{array}{l}\text { 4. Page 8, } \\
\text { 3rd Bullet }\end{array}$ & & $\begin{array}{l}\text { No letter of closure will be issued until the Corrective Action Plan for } \\
\text { closure and the work is completed. }\end{array}$ & \multicolumn{2}{|c|}{$\begin{array}{l}\text { No corrective action plan is necessary because closure activities } \\
\text { have been completed and reported in this CADD/CR. }\end{array}$} & Yes \\
\hline $\begin{array}{l}\text { 5. Page 8, } \\
\text { 4th Bullet }\end{array}$ & & $\begin{array}{l}\text { CAU } 266 \text { will be moved from Appendix III to Appendix IV only when } \\
\text { the Corrective Action Plan for closure and the work is completed. }\end{array}$ & \multicolumn{2}{|c|}{$\begin{array}{l}\text { No corrective action plan is necessary because closure activities } \\
\text { have been completed and reported in this CADD/CR. }\end{array}$} & Yes \\
\hline $\begin{array}{l}\text { 6. Page A-2, } \\
\text { 2nd Paragraph, } \\
\text { 3rd Sentence }\end{array}$ & & $\begin{array}{l}\text { "... The subsurface soils in the vicinity of the collection system and } \\
\text { leachfield may have been impacted by radioactive and other } \\
\text { contaminants of potential concern (COPCs).... NDEP was of the } \\
\text { opinion that the investigation conducted should have resolved this } \\
\text { question. If this is not the case, then this site cannot be closed at } \\
\text { this time. }\end{array}$ & \multicolumn{2}{|c|}{$\begin{array}{l}\text { This sentence was changed to read "This CAU was investigated } \\
\text { because process knowledge indicated that the subsurface soils } \\
\text { in the vicinity...." }\end{array}$} & Yes \\
\hline
\end{tabular}


NEVADA ENVIRONMENTAL RESTORATION PROJECT

\section{DOCUMENT REVIEW SHEET}

\begin{tabular}{|c|c|c|c|c|}
\hline $\begin{array}{l}\text { 10. Comment } \\
\text { Number/ } \\
\text { Location }\end{array}$ & 11. Type* & 12. Comment & 13. Comment Response & $\begin{array}{c}14 . \\
\text { Accept }\end{array}$ \\
\hline $\begin{array}{l}\text { 7. Page A-6, } \\
\text { Section A.2.3, } \\
\text { 1st Paragraph, } \\
\text { Last Sentence }\end{array}$ & & $\begin{array}{l}\text { "...No elevated radiation (using the Electra)... were detected within } \\
\text { the pipes near the openings...". This implies a physical sample was } \\
\text { taken and presented to the Electra. Please clarify. }\end{array}$ & $\begin{array}{l}\text { No sampling was conducted during Stage II (video mole survey) } \\
\text { activities. The Electra and PID were used to monitor within the } \\
\text { pipes at the openings or access points only. The sentence was } \\
\text { not modified. }\end{array}$ & No \\
\hline 8. Page A-44 & & $\begin{array}{l}\text { Dose and Risk Calculation Methodology: The risk models and dose } \\
\text { conversion factors (DCFs) used are from FGR-13. According to } \\
\text { DOE Order } 5400.5 \text {, Radiation Protection of the Public and the } \\
\text { Environment, page 4, paragraph y, the preferred DCFs for use by } \\
\text { Federal agencies is FGR-11. Until this policy statement changes, all } \\
\text { dose and risk calculations should employ the FGR-11 DCFs. }\end{array}$ & $\begin{array}{l}\text { No change made. The risk models and dose conversion factors } \\
\text { (DCFs) used were from FGR-13 and ICRP } 68 \text { and are } \\
\text { presented in Attachment } 1 \text { of Appendix A. In response to the } \\
\text { use of FGR } 11 \text {, the DOE Order } 5400.5 \text {, Page } 4 \text { citation is from } \\
\text { the "references" section and only included because those are } \\
\text { the DCFs presented in Chapter III. DOE Order } 5400.5 \text {, Chapter } \\
1 \text {, Section } 10 \text {, states that the applicable DCFs should be used } \\
\text { when performing calculations of dose from exposures to the } \\
\text { public. Using this as guidance, the use of FGR } 13 \text { is appropriate. } \\
\text { DOE Order } 5400.5 \text {, Chapter IV specifically references } \\
\text { DOE/CH/ } 8901 \text { (RESRAD user manual by Gilbert et al., 1989) as } \\
\text { a guidance to be used for calculating the residual radioactive } \\
\text { material guidelines. This document stated that the ICRP } \\
\text { effective dose model shall be used for deriving soil } \\
\text { concentration guidelines. The most recent ICRP dose } \\
\text { conversion factors are those listed in ICRP } 68 \text { and } 72 \text {. The } \\
\text { senior author of FGR } 11 \text { and } 12 \text {, Keith Eckerman, stated that } \\
\text { FGR } 11 \text { and } 12 \text { are to be revised to incorporate the dosimetry } \\
\text { models of ICRP } 68 \text {. Other DOE contractors and NRC licensees } \\
\text { are currently using ICRP } 68 \text {. Additionally, FGR } 13 \text { makes use of } \\
\text { the most up-to-date scientific information available for risk } \\
\text { assessment. FGR } 13 \text { incorporates the most recent } \\
\text { age-dependent dose model published by ICRP, EPAs current } \\
\text { radiation risk models, and the most recent U.S. decennial } \\
\text { census and cancer models. }\end{array}$ & No \\
\hline
\end{tabular}

a Comment Types: $M=$ Mandatory, $S$ = Suggested.

Return Document Review Sheets to DOE/NV Environmental Restoration Division, Attn: QAC, M/S 505. 


\section{Distribution}

\section{Copies}

* Provide copy in distribution of Revision 0 and subsequent revisions if applicable. Copies of the NDEP approved document will be distributed to others.

Paul J. Liebendorfer

State of Nevada

Bureau of Federal Facilities

Division of Environmental Protection

333 W. Nye Lane, Room 138

Carson City, NV 89706-0851

Michael McKinnon

State of Nevada

Bureau of Federal Facilities

Division of Environmental Protection

555 E. Washington, Suite 4300

Las Vegas, NV 89101

Sabrina Lawrence

Environmental Restoration Division

DOE/Nevada Operations Office

P.O. Box 98518, M/S 505

Las Vegas, NV 89193-8518

Janet Appenzeller-Wing

Environmental Restoration Division

DOE/Nevada Operations Office

P.O. Box 98518, M/S 505

Las Vegas, NV 89193-8518

Clayton Barrow

Environmental Restoration Division

DOE/Nevada Operations Office

P.O. Box 98518, M/S 505

Las Vegas, NV 89193-8518

Wayne Johnson

Bechtel Nevada

P.O. Box 98521, M/S NTS306

Las Vegas, NV 89193-8521
2 (Controlled)*

1 (Controlled)*

1 (Controlled)*

1 (Uncontrolled)*

1 (Uncontrolled)*

1 (Uncontrolled)* 
Jerry Bonn

Bechtel Nevada

P.O. Box 98521, M/S NTS306

Las Vegas, NV 89193-8521

IT Corporation Central Files

P.O. Box 93838

Las Vegas, NV 89193

Robert McCall

SAIC

P.O. Box 93838

Las Vegas, NV 89193

Dustin Wilson

SAIC

P.O. Box 93838

Las Vegas, NV 89193

Manager Southern Nevada FFACO

Public Reading Room

P.O. Box 98521, M/S NLV040

Las Vegas, NV 89193-8521

Manager Northern Nevada FFACO

Public Reading Room

c/o Rosa Silver

IT Corporation

P.O. Box 93838

Las Vegas, NV 89193

FFACO Public Reading Room Coordinator

Rosa Silver

IT Corporation

P.O. Box 93838

Las Vegas, NV 89193

Technical Information Resource Center

DOE/Nevada Operations Office

P.O. Box 98518, M/S 505

Las Vegas, NV 89193-8518
1 (Uncontrolled)*

1 (Uncontrolled)*

1 (Uncontrolled)*

1 (Uncontrolled)*

1 (Controlled)

1 (Uncontrolled)

1 (Uncontrolled)

1 (Controlled)

1 (Uncontrolled) 
U.S. Department of Energy

Office of Scientific and Technical Information

P.O. Box 62

Oak Ridge, TN 37831
1 (Uncontrolled, electronic copy) 